\title{
SULL'ORIGINE DEI MICROSISMI \\ CON PARTICOLARE RIGUARDO ALL'ALTO ADRIATICO (*)
}

\author{
Pietro Calor
}

1. - Nello studio dei microsismi, ci si è generalmente limitati alle tempeste associate a grandi cicloni, interessanti estese aree oceaniche e continentali. Ritengo che sia questa la via meno facile per pervenire a conclusioni attendibili. I grandi cicloni sono sempre collegati allo scatenarsi di forze di diversa natura, quali i venti e l'urto dei marosi. Le variazioni rapide della pressione, nel caso dei grandi cicloni, non sono mai disgiunte dalle altre due cause perturbanti: il vento e il mare fortemente agitato. Ben difficilmente è possibile sceverare, in questi casi, l'azione di una causa da quella di un'altra.

Ritengo possa essere di interesse, a questo fine, portare la propria attenzione a tempeste microsismiche, anche di piccola entità, associate a piccoli cicloni o anche al semplice transito di lievi disturbi microbarici, interessanti zone molto limitate.

Particolarmente idoneo per questo genere di ricerche, si presenta l'alto Adriatico, specie nel tratto compreso fra le coste del Veneto e dell'Istria. Questo tratto di mare è interessato dalle piccole formazioni cicloniche che dalla Valle Padana si spostano verso la Balcania. Sulle sponde opposte funzionano delle ottime stazioni d'osservazione, tanto dal punto di vista meteorologico, quanto da quello sismico che dello stato del mare. Particolarmente attrezzati a questo riguardo gli Osservatori geofisici del Magistrato alle Acque e del Seminario Patriarcale di Venezia, e dell'Istituto Talassografico e dell'Osservatorio Geofisico di Trieste. Le cause principali, a cui sono attribuiti i microsismi, e cioè le rapide variazioni di pressione, il mare agitato e il vento, possono essere registrate, nella loro entità, sulle opposte rive del tratto di mare accennato (fig. 1).

Ciò costituisce una condizione nettamente favorevole per lo studio dei microsismi, che ben difficilmente può essere realizzata in altre situazioni.

* Largo riassunto di una comunicazione presentata alla IX Assemblea Generale dell'U.G.G.I., tenutasi a Bruxelles dal 19 al 31 agosto 1951. 
Ho raccolto molti esempi di registrazioni microsismiche, ottenute a Trieste e a Venezia in epoche diverse. Da tali esempi, che verranno riassunti in una puhhlicazione più dettagliata, risultano chiare due cause di microsismi nell'alto Adriatico: 1) il passaggio sul mare, da Ovest ad Est, di rapide perturbazioni della pressione atmosferica, legate oppure no a dei cicloni; 2) l'azione sul fondo dei sistemi d'onde,

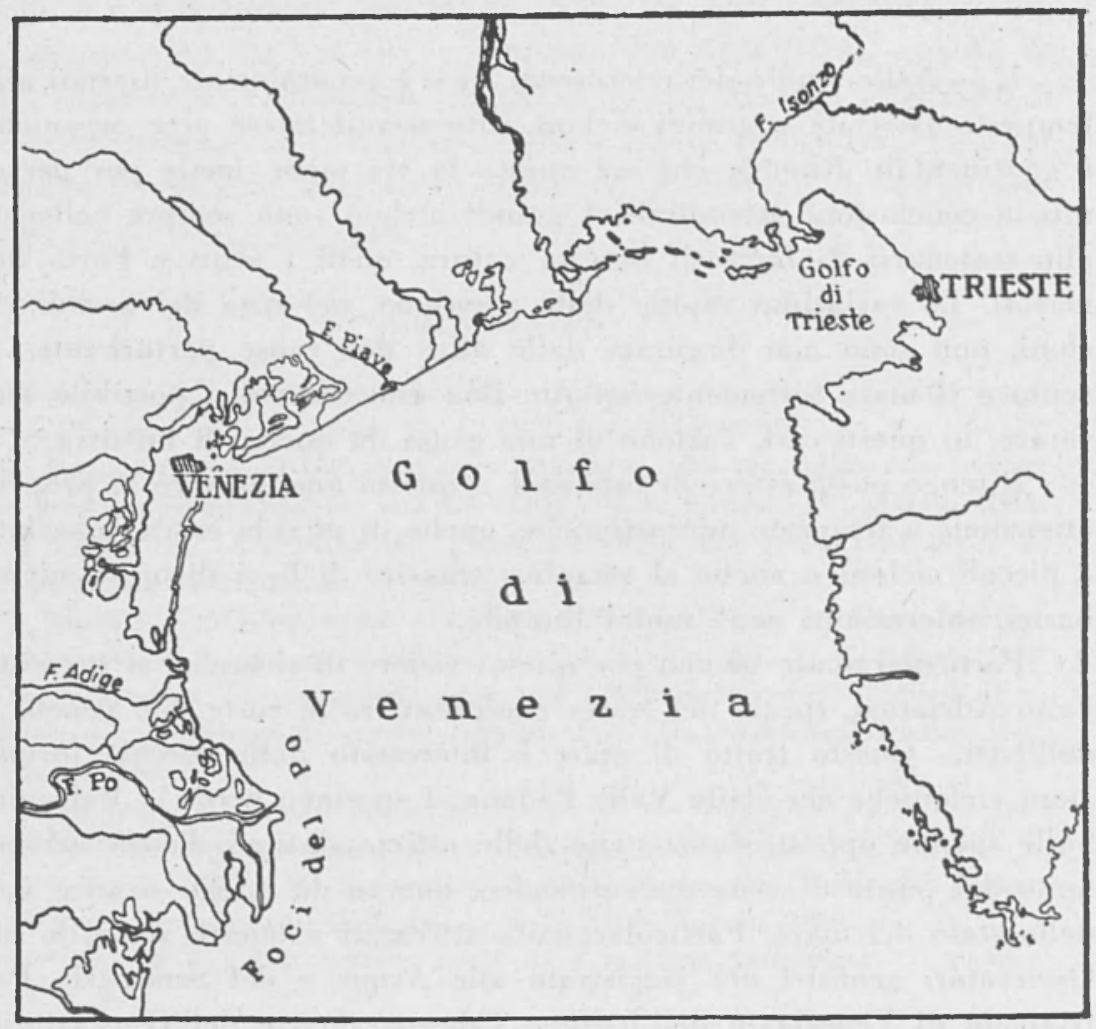

Fig. 1

determinati alla superficie del mare dal vento soffiante, per parecchie ore, nella stessa direzione.

Fermiamoci un po' sulla prima causa.

Fin dal 1936 ebbi modo di provare $\left({ }^{1}\right)$ che, a Trieste, notevoli movimenti microsismici, con periodi da 2 a 3 sec., erano associati alla brusca ripresa della pressione atmosferica a Trieste. Per quanto concerne l'esempio del 13 ottobre 1933, così mi esprimevo: "Alle $13^{\text {h }}$ bruscamente, come è provato dalla rapida salita del barometro a Trie- 
ste, il ciclone comincia la sua deriva verso SE...; nello stesso tempo i microsismi, regolarissimi, raggiungono ampiezze notevoli, pur conservando il periodo $"$.

E a proposito dell'altro ciclone del 16 ottobre 1934, che verrà discusso più avanti, ecco le mie osservazioni: "Verso le $4^{\text {h }}$ del 16 il centro della depressione.... inizia la sua rotta sud-orientale, in modo piuttosto brusco; subito i microsismi a breve periodo $\left(3^{\mathrm{B}} \mathrm{ca}\right.$.) sulle componenti $\mathrm{EW}$, NS aumentano di ampiezza notevolmente ».

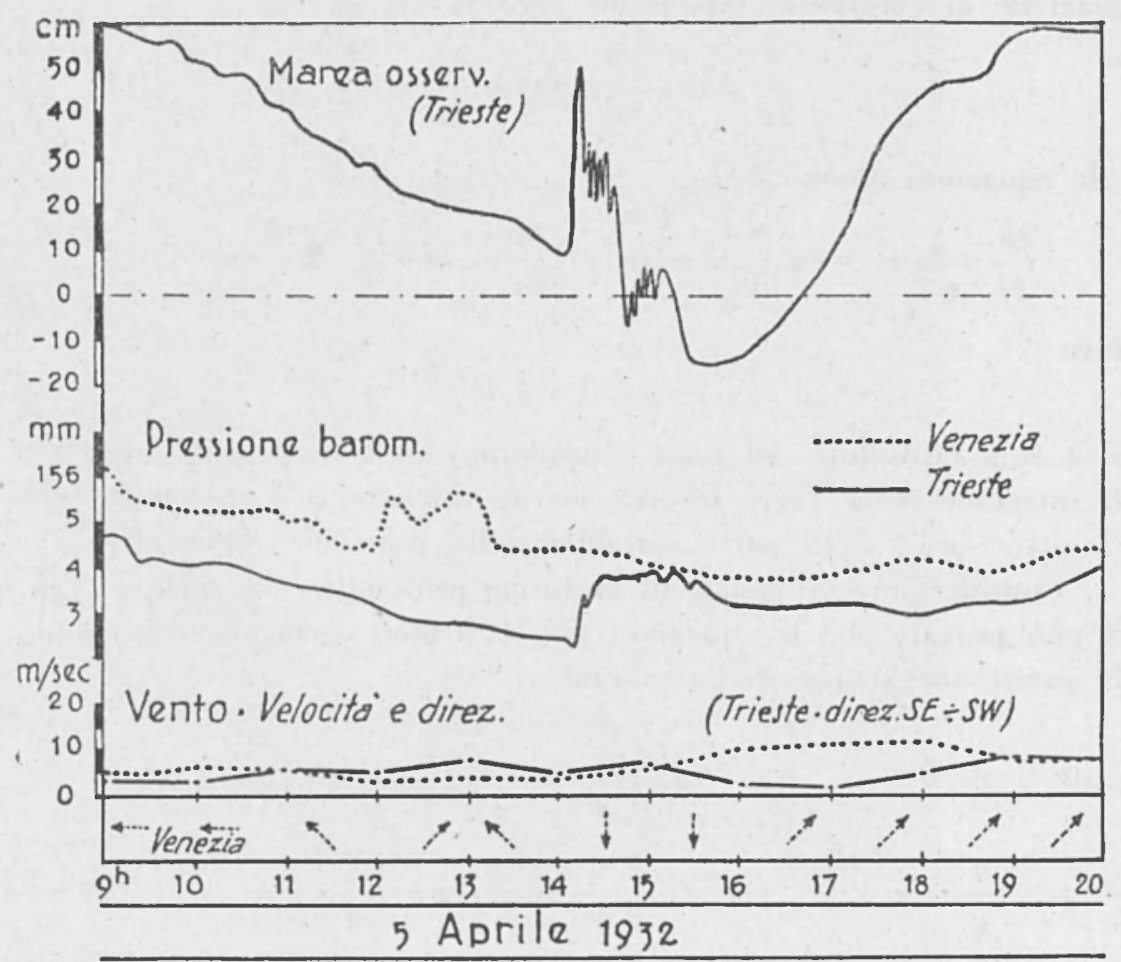

Fig. 2

Nell'alto Adriatico, dunque, microsismi di sensibile ampiezza possono essere associati alla fase positiva di un ciclone in transito o semplicemente al passaggio di perturbazioni microbarometriche, propagantisi dall'Ovest all'Est con velocità determinata. Vedremo come, a nostro avviso, ciò possa verificarsi.

Richiamo, a questo punto, una teoria di Proudman sugli effetti delle variazioni di pressione atmosferica sui moti superficiali del mare $\left({ }^{2}\right)$. 
Se $\omega$ è la componente, secondo la verticale, della velocità angolare di rotazione della terra; $g$ l'accelerazione di gravità; $h$ la profondità del mare in un punto; $t$ il tempo; $x, y$ le coordinate cartesiane in un punto coincidente con la superficie indisturbata del mare; $u$ e $v$ le componenti della corrente nella direzione $x, y$, in un punto $e$ in un determinato tempo; $\zeta$ l'elevazione della superficie libera del mare sopra il livello medio; $-\bar{\zeta}$ la parte variabile in altezza corrispondente alla variazione barometrica ("sea-water barometer"), l'equazione di continuità può essere scritta:

$$
\frac{\partial}{\partial x}(h u)+\frac{\partial}{\partial y}\left(h_{\bullet} v\right)+\frac{\partial \zeta}{\partial t}=0
$$

e le equazioni dinamiche

$$
\frac{\partial u}{\partial t}-2 \omega v=-g \frac{\partial}{\partial x}(\zeta-\bar{\zeta}) \quad ; \quad \frac{\partial v}{\partial t}+2 \omega u=-g \frac{\partial}{\partial y}(\zeta-\bar{\zeta})
$$

dove

$$
\omega=\Omega \sin \lambda
$$

se $\lambda$ è la latitudine del mare considerato e $\Omega$ la velocità angolare di rotazione della Terra intorno al suo asse. Se $\varrho$ è la densità dell'acqua, $-g_{\varrho} \zeta$ è la parte variabile della pressione atmosferica.

Consideriamo un canale di uniforme profondità $h$ e sia $c=\mid \overline{g h}$. Si può provare che le equazioni [1], [2] sono verificate, da ognuno di questi due gruppi di espressioni:

$$
\begin{aligned}
& |\bar{\zeta}=0, v=0 \quad| \frac{\bar{\zeta}}{c^{2}}=e^{-2 \omega y / V} F(x-V t) \\
& \frac{\zeta}{c}=\frac{u}{g}=e^{\mp 2 \omega y c} f(x \mp c t) \mid \frac{\zeta}{c^{2}}=\frac{u}{V g}=\frac{1}{1-V / c^{2}} e^{-2 \omega y / V} F(x-V t)
\end{aligned}
$$

dove $V$ è una costante e $f(\ldots), F(\ldots)$ rappresentano funzioni qualunque dei loro argomenti. Tali soluzioni rappresentano perturbazioni propagantisi con velocità uniforme, parallela all'asse delle $x$.

La presenza del fattore $1 /\left(1-\mathrm{V}^{2} / \mathrm{c}^{2}\right)$ in [3] indica una grandissima perturbazione dell'acqua quando la perturbazione atmosferica viaggia con velocità uguale a quella propria delle libere perturbazioni dell'acqua.

Per semplificare la trattazione, che ha carattere puramente qua- 


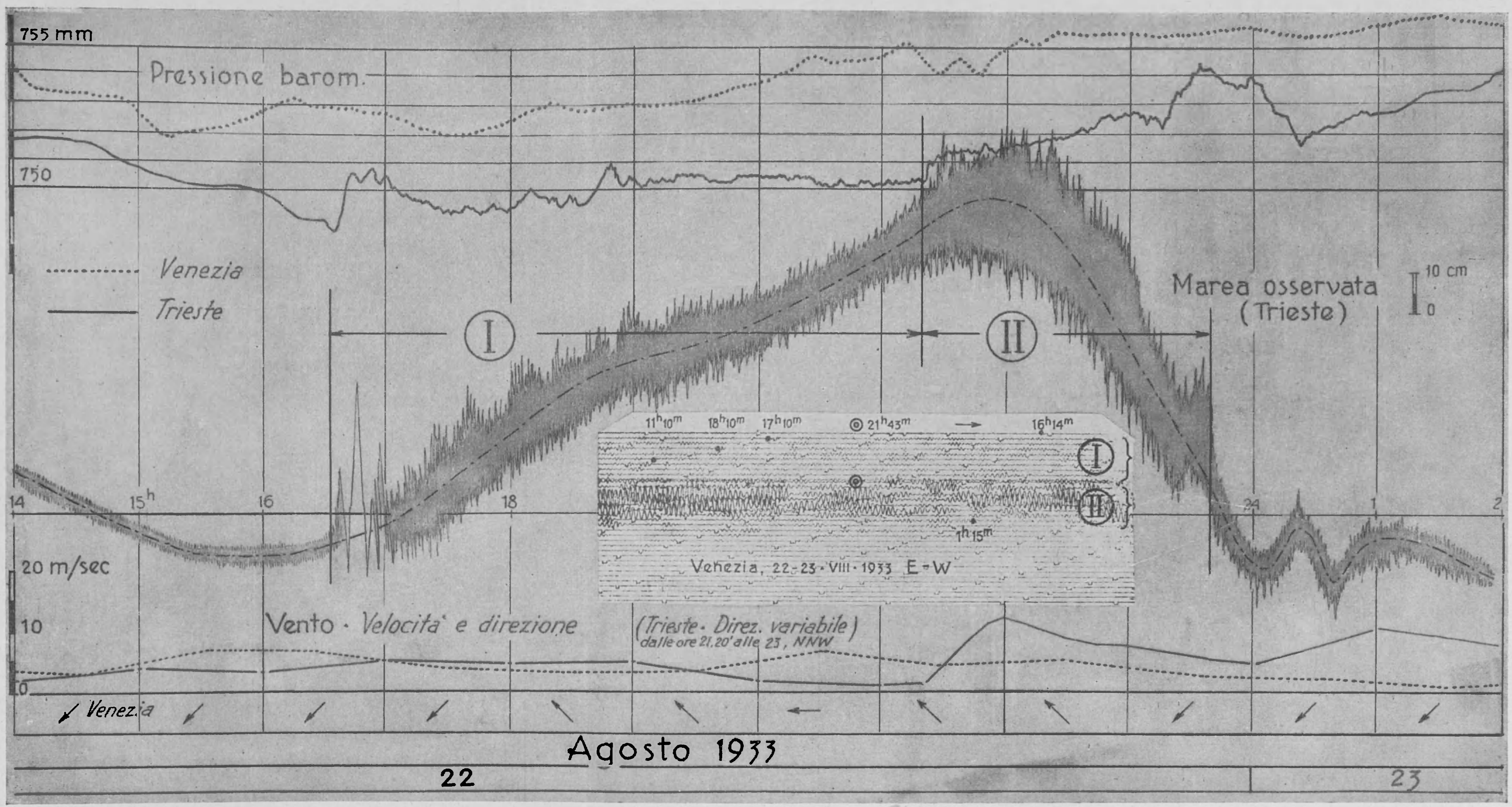

Fig. 3 - Condizioni atmosferiche a Trieste e a Venezia, stato del mare a Trieste e microsismi a Venezia il 22 e 23 agosto 1933. (Nel sismogramma di Venezia leggere $19^{h} 10^{\mathrm{m}}$ anziché $11^{\mathrm{h}} 10^{\mathrm{m}}$ ). 

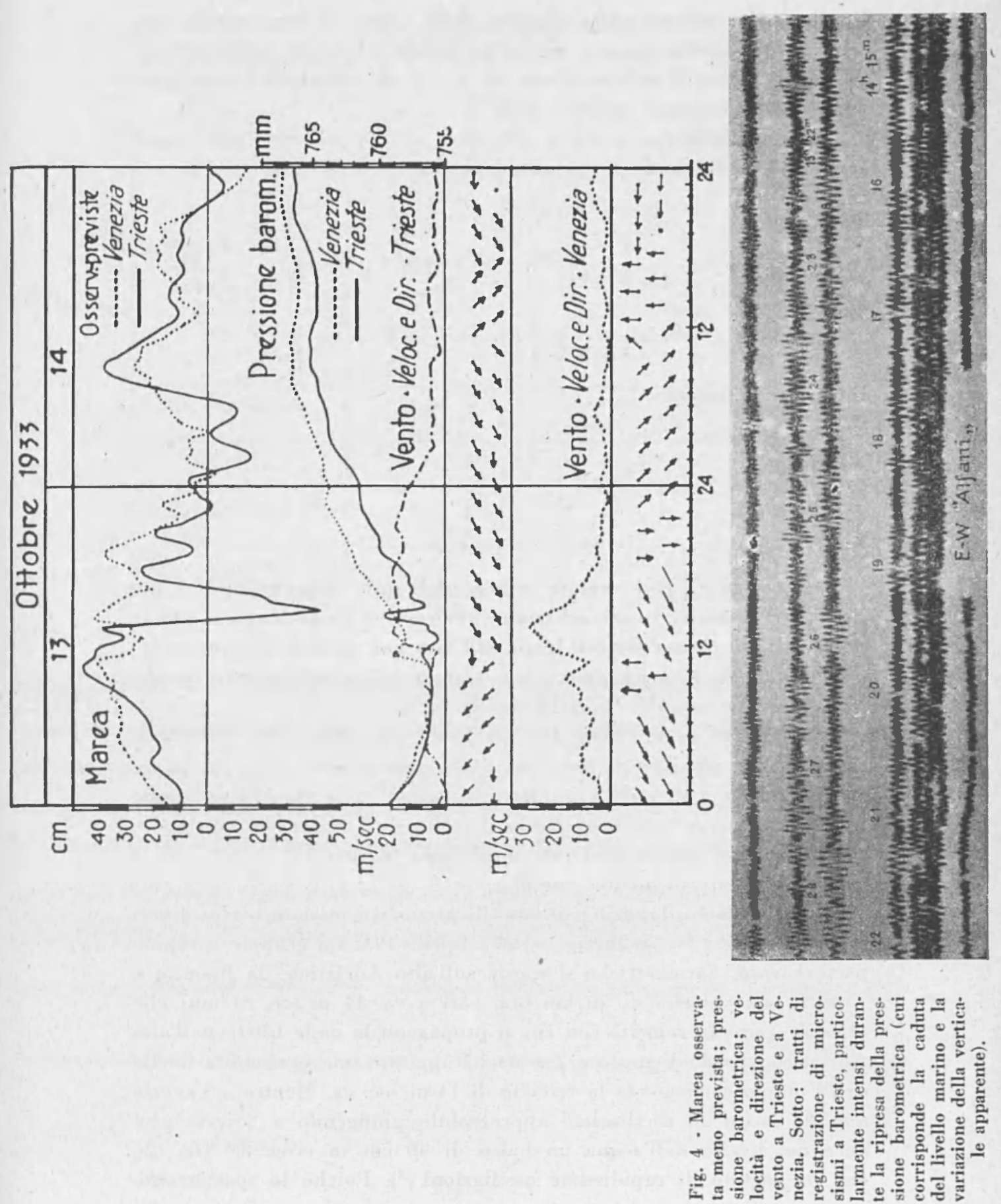
litativo, prescindiamo dalla rotazione della Terra (il che equivale considerare uno stretto canale; oppure un canale nei pressi dell'equatore).

Supponiamo il canale chiuso ad $x=0$ ed estendentisi indefinitamente nella direzione positiva delle $x$.

Allora dobbiamo avere $u=0$ dove $x=0$, ciò che puó essere assicurato combinando le [3] come segue:

$$
\begin{aligned}
& \zeta=-(t-x / V), v=0 \\
& \zeta=\frac{1}{1-V^{2} / c^{2}}\{F(t-x / V)-v / c F(t-x / c)\} \\
& \frac{c}{g} u=\frac{V / c}{1-V^{2} / c^{2}}\{F(t-x / V)-F(t-x / c)\}
\end{aligned}
$$

Dove $x=0$, abbiamo

$$
\zeta=\frac{1}{1+V / c} F(t)
$$

così che

$$
\frac{\zeta}{\zeta}=\frac{1}{1+v / c}
$$

La costante [5] è sempre minore dell'unità fintantoché $V$ è positivo, cioè quando la perturbazione atmosferica passa lungo il canale dall'estremità chiusa verso il largo. Ma essa può assumere valori molto grandi quando $V$ è negativo, ciò̀ quando la perturbazione atmosferica si muove verso l'estremità chiusa.

In tal caso, se in valore assoluto $V$ e $c$ coincidono, la [5] diviene teoricamente infinita.

Potrei dare un grande numero di esempi sulla validità di questa legge, almeno per quanto riguarda il Golfo di Trieste, che riproduce, con sufficiente approssimazione, il modello teorico.

Mi limiterò ad alcuni esempi.

Consideriamo dapprima il caso di perturbazioni non legate a veri e propri cicloni. Nel pomeriggio del 5 aprile 1932 un gruppo di rapide perturbazioni barometriche si sposta sull'alto Adriatico, da Venezia a Trieste, con velocità di $50 \mathrm{~km} /$ ora, pari a ca. $14 \mathrm{~m} / \mathrm{sec}$. Si noti che questa è pure la velocità con cui si propagano le onde libere nell'alto Adriatico, che da Venezia a Trieste ha appunto una profondità media di $20 \mathrm{~m}$, cui corrisponde la velocità di $14 \mathrm{~m} / \mathrm{sec}$. ca. Mentre a Venezia non dà luogo ad oscillazioni apprezzabili, giungendo a Trieste provoca nel livello dell'acqua un balzo di $40 \mathrm{~cm}$ in circa $8^{\mathrm{m}}$ (fig. 2), con un seguito di rapidissime oscillazioni $\left({ }^{3}\right)$. Poiché lo spostamento 


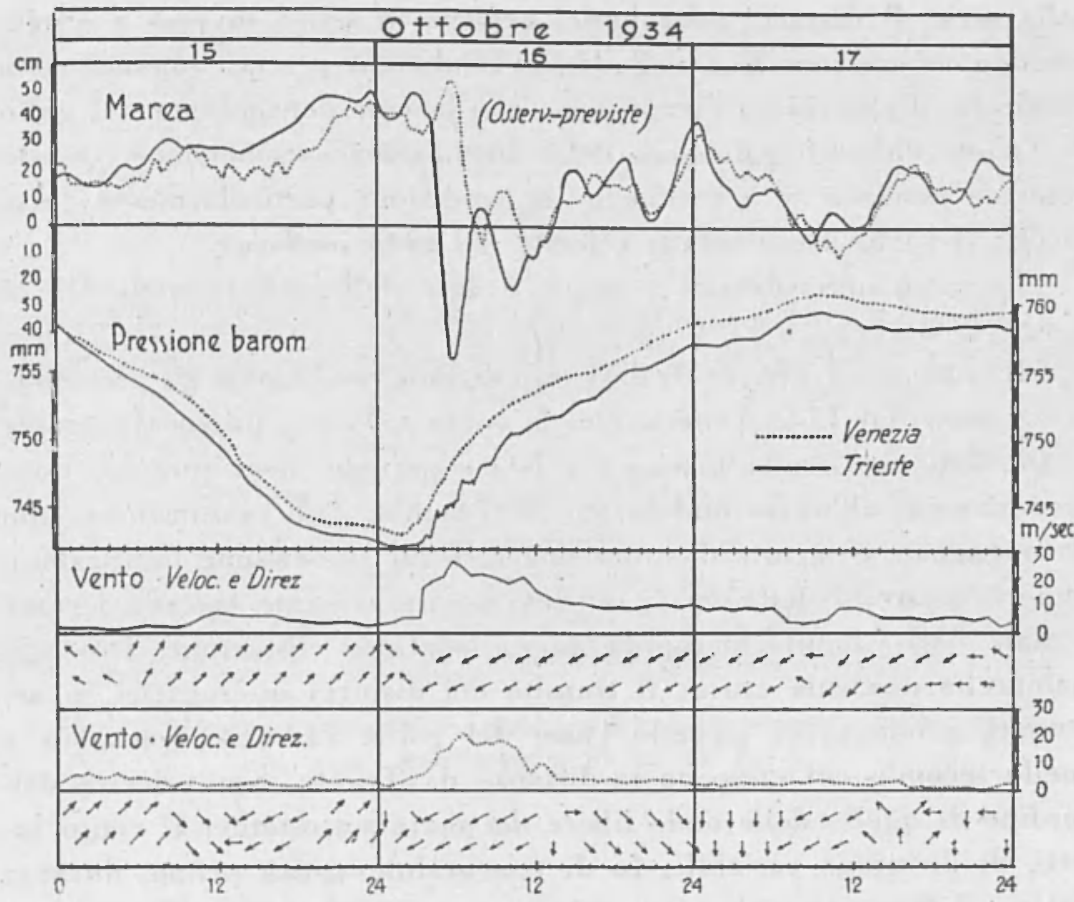

Fig. 5

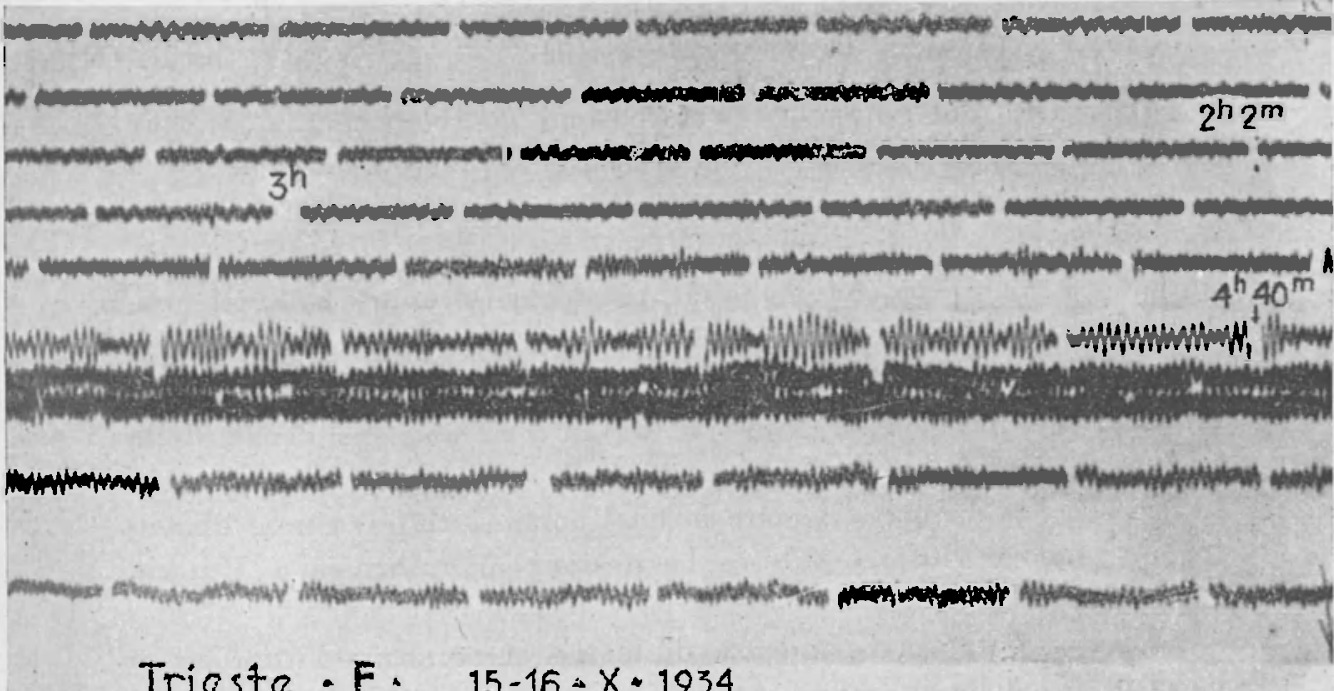

Trieste : E E E

ijg. 6 
della serie di disturbi microbarici avviene in senso inverso a quello secondo cui crescono le $x$, nella [5] la condizione per la risonanza resta verificata, il che spiega l'amplissima oscillazione determinata nel golfo di Trieste dal sopraggiungere delle fluttuazioni barometriche. Questo notevole esempio si è verificato in condizioni particolarmente favorevoli : il vento aveva infatti velocità del tutto inefficace.

Un altro notevolissimo esempio è dato dalle registrazioni del 22 agosto 1933 .

Il transito su Trieste di lievi, rapidissime oscillazioni di pressione, provenienti dal Lido Veneto, con la solita velocità, provocava ampie oscillazioni del livello marino $\left({ }^{3}\right)$ di un periodo assai piccolo, dove predominano all'inizio ondulazioni di $7.8 \mathrm{~min}$. Tali ondulazioni sono accompagnate e seguite da tutta una serie di rapidissime oscillazioni, come è mostrato dalla fig. 3 . È davvero interessante osservare come il mare sia ivi tenuto in rapida, ampia, continua vibrazione. Tale agitazione ha una sola causa: il transito dei disturbi microgarici su accennati, propagantisi secondo l'asse del golfo, in senso contrario a quello secondo cui crescono le distanze da Trieste, con velocità dell'ordine di quella delle onde libere del mare sottostante. Il vento infatti, di direzione variabile, fu di trascurabile entità prima, durante e dopo il fenomeno, che non può essere stato comunque da esso influenzato.

Vediamo come procede il fenomeno dal punto di vista dei microsismi. Mi riferisco alla registrazione di Venezia.

Il transito del gruppo di perturbazioni microbariche su Venezia non determina ivi agitazione microsismica apprezzabile.

Non appena il gruppo di perturbazioni perviene sul golfo di Trieste $\left(16^{\mathrm{h}} 30^{\mathrm{m}}\right.$ ca.), l'agitazione microsismica a Venezia si fa subito sensibile e si mantiene con ampiezza pressoché costante, in corrispondenza della rapida agitazione, ad ampiezza pure pressoché costante, determinata nel mare del golfo di Trieste dal gruppo di perturbazioni atmosferiche in transito. Verso le $21^{\mathrm{h}} 20^{\mathrm{m}}$, in corrispondenza della brusca ripresa delle rapide perturbazioni atmosferiche di passaggio su Trieste, l'agitazione del mare in quel porto si rinforza notevolmente: un analogo rinforzo subisce l'agitazione microsismica a Venezia (fig. 3).

Una perfetta corrispondenza di tempo accompagna l'insorgere e l'accentuarsi delle agitazioni marina e microsismica, determinate come immediata e mediata conseguenza del transito dei disturbi microbarici, obbediente alla [5]. 


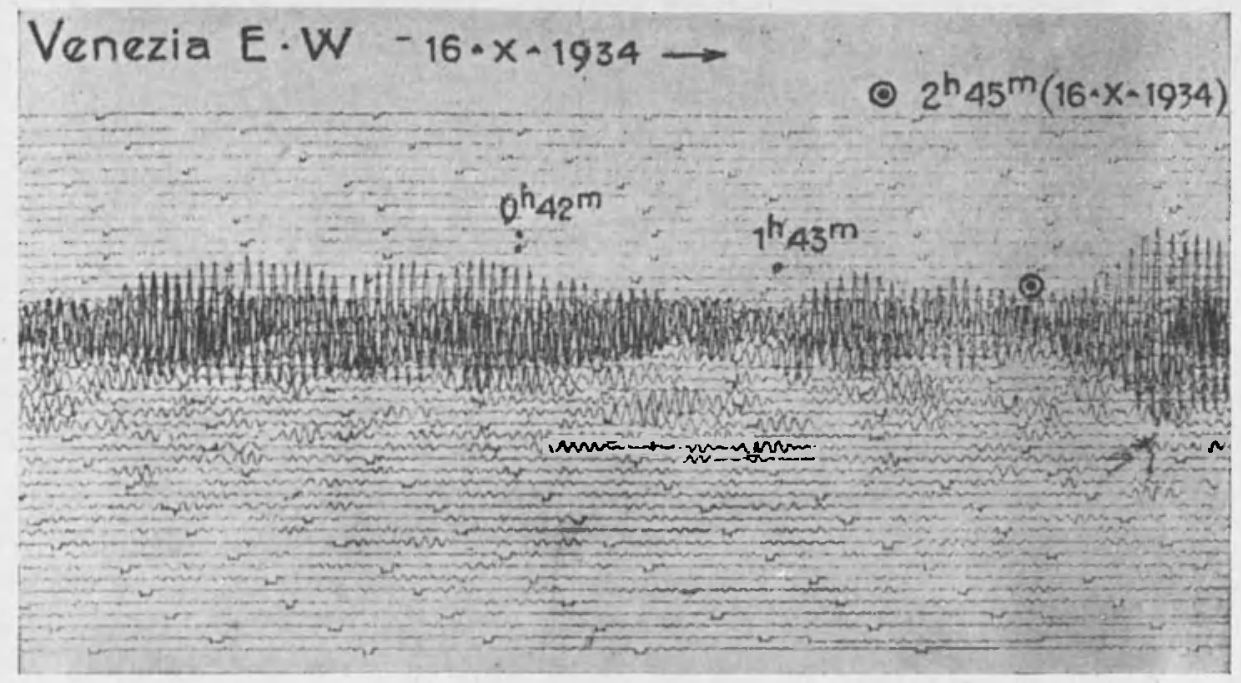

Fig. 7 - Agitazione microsismica registrata a Venezia in concomitanza alla situazione meteorologica di cui alla fig. 5

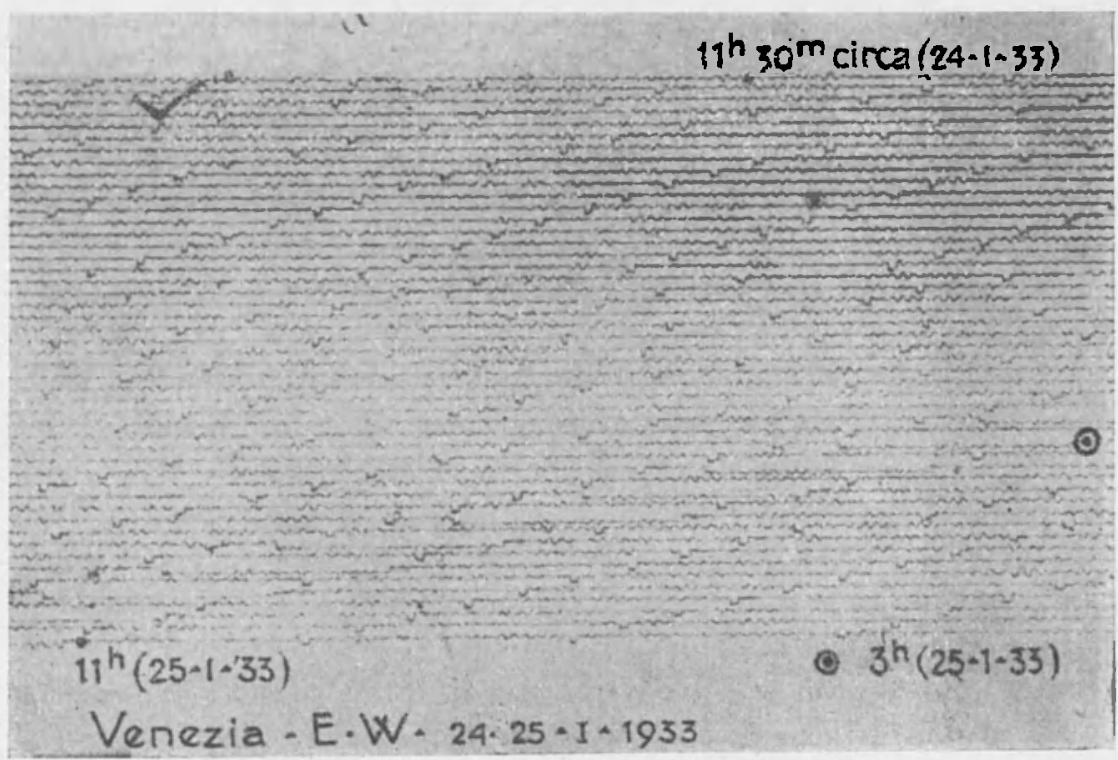

Fig. 8 - Agitazione microsismica a Venezia determinata da bora anticiclonica con velocità media di $15.20 \mathrm{~m} / \mathrm{sec}$ 
Quello citato costituisce un esempio sorprendente, d'interesse a mio parere eccezionale, per ciò che concerne l'origine dei microsismi accennati: la causa determinante vi è qui individuata ed isolata senza possibilità di equivoci. Ciò che, a mio avviso e per quanto mi consta, costituisce un fatto nuovo, del quale non va sottovalutata l'importanza.

Avrei diecine e diecine di altri esempi in testimonianza della validità della [5]. Ritengo superfluo citarli.

Dirò, invece che, nel caso del transito di cicloni osservanti la legge più volte citata, allora, la maggior energia in gioco, determina oscillazioni del mare molto più ampie $e$, molto spesso, desta la sessa fondamentale del golfo, avente un periodo di $3^{\text {h }}, 5$ ca. Anche qui potrei citare molti esempi: mi limiterò a quelli del 13 ottobre 1933 e del 16 ottohre 1934. Qui, la brusca ripresa della pressione sul golfo di Trieste è accompagnata da tutta una serie di variazioni barometriche, comprese le più rapide. Le più lente, come si ̀̀ detto, causano la sessa del gollo, e, nella terra terma, la variazione della verticale apparente, registrata dalla compo-

Fig. 9 nente $\mathrm{EW}$ del foto-sismografo "Alfani », sotto forma d'allontanamento e successivo riavvicinamento delle linee. Alle più rapide sono senz'altro da ascriversi i microsismi registrati, in tali occasioni, a Trieste e a Venezia.

Si noti infatti l'esempio del 13 ottobre 1933 a Trieste: $\dot{e}$ durante la rapida caduta del mare, causata dalla brusca ripresa della pressione a Trieste, che si registrano $i$ massimi microsismi: $\grave{e}$ in questo periodo quindi, che riesce massima sul fondo l'azione dell'energia, legata ai disturbi microbarici della pressione in ripresa.

La situazione meteorologica durante la giornata è indicata dalla fig. 4 .

Per il caso considerato mancano le registrazioni di Venezia. Dalla fig. 4 risulta che la perturbazione ciclonica passa su Venezia verso le $10^{\text {h }}$. A partire da questa ora, il nucleo ciclonico procede al di sopra del mare, verso Trieste. Un'agitazione leggerissima è registrata dagli "Alfani " di Trieste, con periodi dell'ordine di 1,5 sec. Verso le $11^{\text {h }}$ e $30^{\mathrm{m}}$, quando la perturbazione è nei pressi del golfo di Trieste, l'agitazione diventa bruscamente più sensibile e la sua ampiezza cresce sempre più e raggiunge il massimo, quando il nucleo della depressione è già passato su Trieste e la pressione è in fase positiva ben netta. L'agitazione tpermane ampia fino alle $16^{\text {h }}$ circa. Resta in seguito una agitazione di fondo che dura a lungo, fino alle prime ore del 15 ottobre. 
SULL'ORICINE DEI MICROSISMI CON PARTICOLARE HIGUARDO ALL'ALTO ADRIATICO 535

15-16 ottobre 1934. La situazione meteorologica sulla Val Padana e sull'alto Adriatico è schematizzata nella fig. 5 , clıe dà l'andamento della pressione a Venezia e a Trieste, nonché la intensità e la direzione del vento a Trieste.

L'agitazione microsismica insorge violenta, e pressoché contemporaneamente verso le $3^{\mathrm{hl}} 50^{\mathrm{m}}$, a Venezia e a Trieste, in corrispondenza del transito del nucleo ciclonico nel golfo di Trieste, come nel caso precedente.

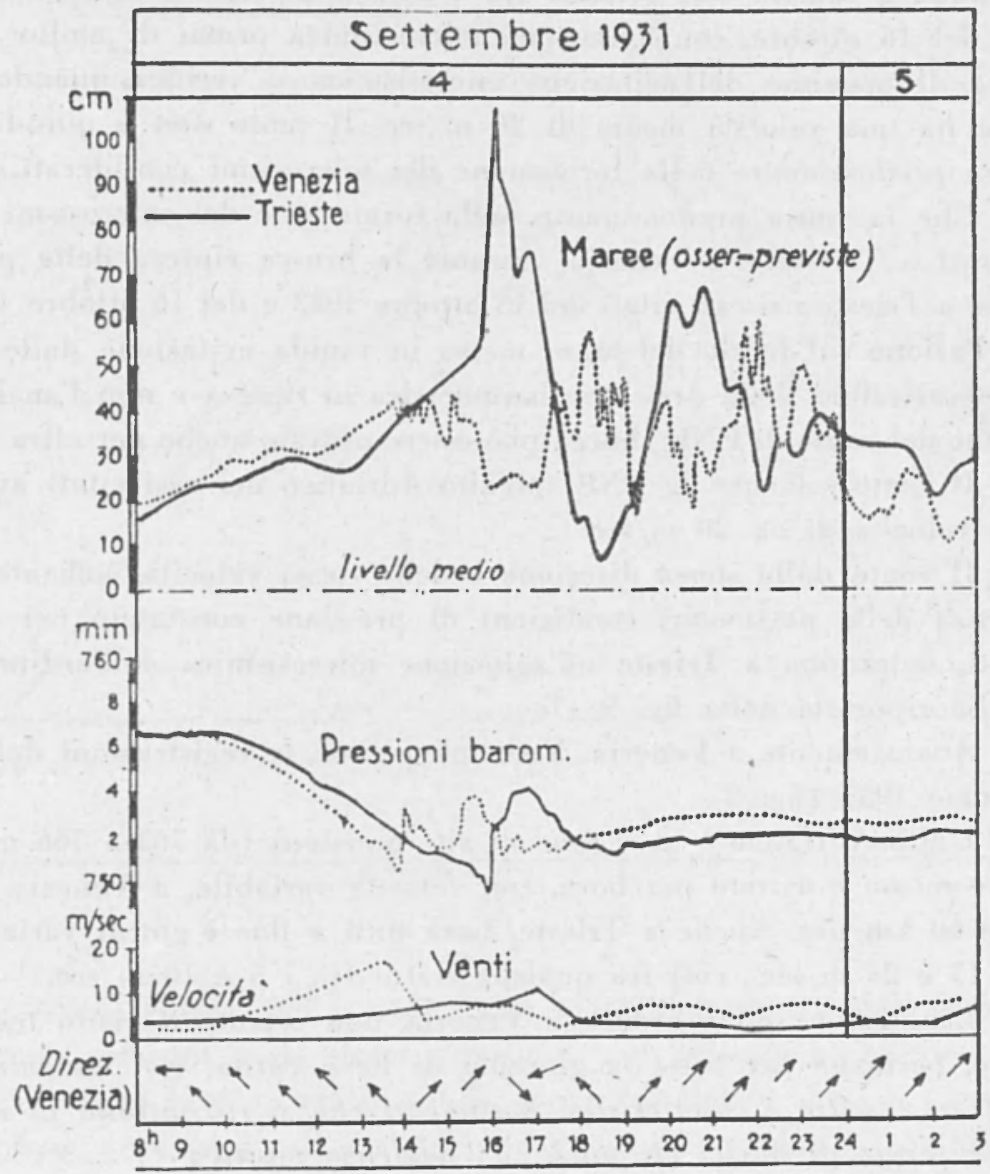

Fig. 10

Va osservato che il centro del ciclone è passato sopra Venezia verso le $2^{\mathrm{h}}$ : a quell'ora, inizia a Venezia la ripresa del barometro senza che ciò provochi microsismi apprezzabili: questi cominciano a rendersi sensibili verso le $3^{\text {h }} 30^{\mathrm{m}}$, quando anche a Trieste la pressione 
è in rapida salita. I massimi, sia a Venezia (fig. 7) che a Trieste (fig. 6), vengono raggiunti dalle $4^{\mathrm{h}} 45^{\mathrm{m}}$ alle $5^{\mathrm{h}} 15^{\mathrm{m}}$ ca. Il periodo inizialmente di 1,5-2 sec. ca., perviene, nel massimo dell'agitazione, intorno a 3 sec.

Alle $3^{\text {h }} 20^{\text {in }}$ ca. con la ripresa della pressione a Trieste, insorge bruscamente la bora (vento da ENE) con velocità di $16 \mathrm{~m} / \mathrm{sec}$. ca.; essa raggiunge il massimo di ca. $25 \mathrm{in} / \mathrm{sec}$, verso le 5,45 , quando l'agitazione microsismica ha già superato il massimo di attività; la bora continua a soffiare con velocità fra i 25 e i $20 \mathrm{~m} / \mathrm{sec}$. fino oltre le $12^{\text {h }}$ del 16 ottobre, con agitazione microsismica ormai di molto scemata. Il massimo dell'agitazione microsismica si verifica quando la bora ha una velocità media di $20 \mathrm{~m}$ 'sec. Il vento non è quindi la causa predominante nella formazione dei microsismi considerati.

Che la causa predominante nella formazione dei microsismi registrati a Trieste e a Venezia, durante la brusca ripresa della pressione a Trieste nei casi citati del 13 ottobre 1933 e del 16 ottobre 1934 sia l'azione sul fondo del mare messo in rapida agitazione dalle rapide variazioni della pressione barometrica in ripresa e non l'analoga azione del vento di ENE (bora), può essere provato anche per altra via.

Il vento soffiante da ENE sull'alto Adriatico nei casi citati aveva una velocità di ca. $20 \mathrm{~m} / \mathrm{sec}$.

Il vento dalla stessa direzione e della stessa velocità, soffiante in assenza delle particolari condizioni di pressione constatato nei casi citati, determina a Trieste un'agitazione microsismica dell'ordine di quella riportata nella fig. $9\left({ }^{4}\right)$.

Analogamente a Venezia. Vediamo per es. le registrazioni del 24 gennaio 1933 (fig. 8).

L'alto Adriatico è in regime di alte pressioni (da 763 a $766 \mathrm{~mm}$ ), mare mosso e agitato per bora, con velocità variabile, a Venezia, fra 50 e $60 \mathrm{~km} /$ ora. Anche a Trieste, bora tutti e due i giorni variabile fra 15 e $25 \mathrm{~m}$ /sec., così fra qualche tratto fra i 5 e $10 \mathrm{~m} / \mathrm{sec}$.

L'agitazione microsismica a Venezia, non ostante il vento teso e forte, permane per tutta la giornata di lieve entità, con andamento uniforme, senza $i$ caratteristici battimenti che si riscontrano in caso di passaggio di nuclei ciclonici, con tendenza positiva $\left(T=3^{\mathrm{s}}\right)$.

Questo è un chiarissimo esempio di agitazione legato a vento (a Venezia, pressione livellata; a Trieste, molto mossa, come sempre in giomi di bora): l'agitazione è dovuta, a mio avviso, ad azione sul fondo del ritmico moto ondoso.

A prescindere da altre considerazioni, va osservato, a questo riguardo, che nei pressi della costa veneta il fondo del mare è a pen- 
denza estremamente dolce, il che rende del tutto improbabile un'azione dinamica dei marosi, sufficiente a determinare dei microsismi apprezzabili. L'onda infatti viene gradualmente frenata nel suo procedere verso la costa e la sua energia gradualmente dispersa per attrito sul fondo.

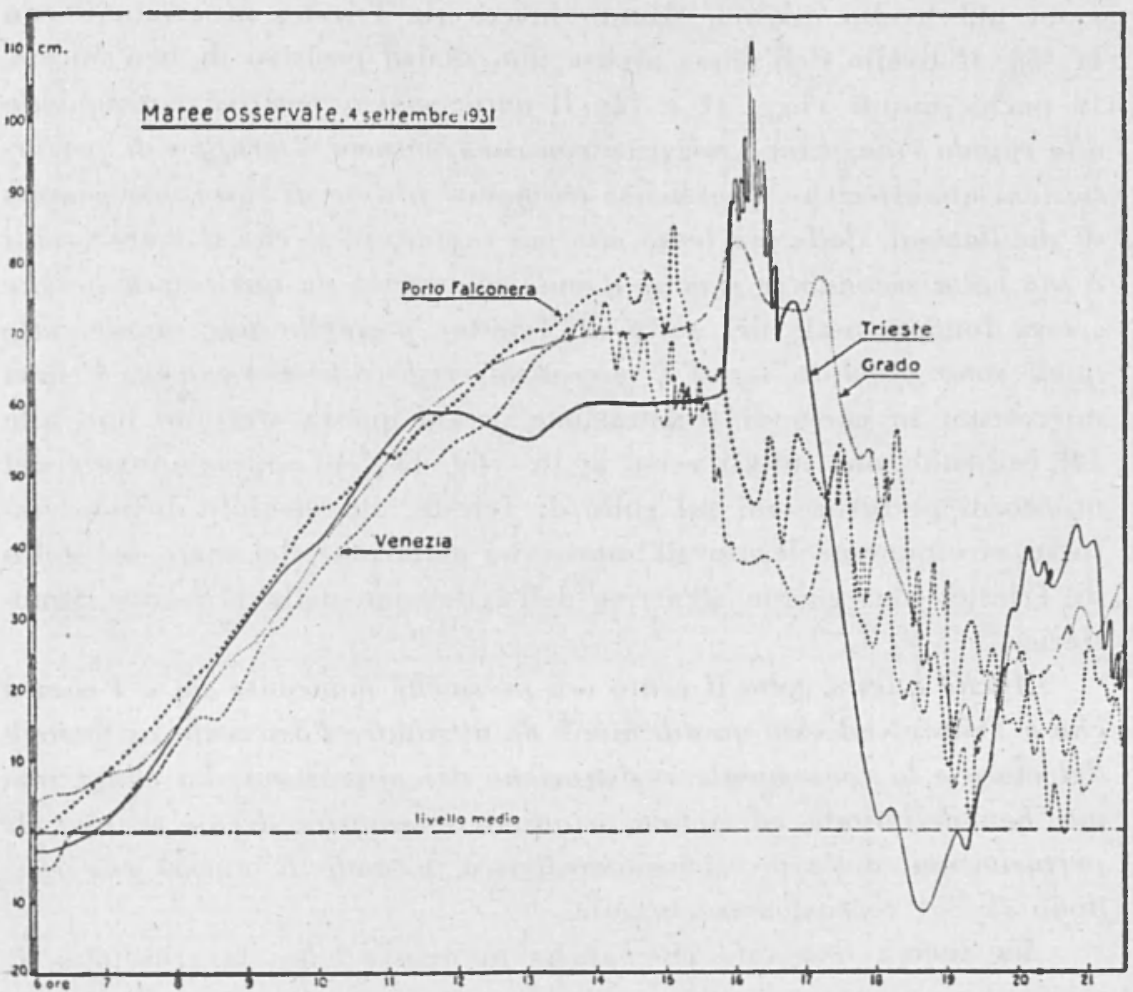

Fig. 11

I sistemi ritmici dei marosi d'alto mare, determinati dall'azione del vento soffiante nella stessa direzione, con velocità pressoché costante, possono invece causare sul fondo, in condizioni speciali (quali appunto si verificano nell'alto Adriatico), ritmiche variazioni di pressione, alle quali i microsismi possono essere attribuiti.

Un altro caratteristico esempio di microsismi legati al transito sul golfo di Trieste di rapide perturbazioni atmosferiche è quello verificatosi il 4 settembre 1931 (fig. 10).

Questo esempio è una riprova chiarissima della validità generale della [5], per quanto riguarda l'agitazione del mare, determinata da 
perturbazioni atmosferiche in transito. Le condizioni da essa richieste sono strettamente verificate per quanto riguarda la direzione e la velocità di transito del nucleo perturbante. Come prova la fig. 11 , il nucleo di perturluazione passando su Venezia, Porto Falconera e Grado, nella sua marcia da $W$ ad $E$, non determina che lievi perturbazioni nel livello marino. Giunto invece su Trieste, in armonia con la [5]. il livello dell'acqua accusa uno sbalzo positivo di ben $50 \mathrm{~cm}$, in pochi minuti (figg. 11 e 12). Il netto sbalzo positivo corrisponde' alla rapida elongazione negativa con cui comincia il gruppo di perturbazioni atmosferiche. L'esistenza fra queste ultime di tutta una gamma di oscillazioni, dalle più lente alle più rapide, fa sì che il mare oscilli a sua volta secondo le gamme d'onda più varie: da quelle più lunghe (sessa fondamentale del golfo di Trieste), a quelle più rapide, alle quali sono appunto legati $i$ microsismi registrati a Venezia. I lievi microsismi in corso di registrazione presso questa stazione fino alle $14^{\text {h }}$ ca., subiscono infatti verso le $16^{\text {h }}$ (fig. 13), col sopraggiungere del nulceo di perturbazioni sul golfo di Trieste, un sensibile, brusco rinforzo, strettamente legato all'improvvisa agitazione del mare nel golfo di Trieste, conseguente all'arrivo dell'agitazione della pressione atmosferica.

Anche questa volta il vento era pressoché mancante sia a Venezia che a Trieste: ad esso quindi non è da attribuire l'osservata agitazione del mare e la conseguente registrazione dei microsismi. La causa pri$m a$, ben delimitata ed isolata, è quindi il transito di un gruppo di perturbazioni della pressione atmosferica, secondo le norme che rendono la [5] teoricamente infinita.

$\mathrm{Va}$ ancora osservato che, anche in questo caso, la creazione di microsismi si è avuta con transito di perturbazioni microbariche legate a pressione in fase stazionaria.

Un'altra osservazione nei riguardi dell'esempio citato. Come avviene aljitualmente, i microsismi registrati a Venezia lo sono contemporaneamente, e con almeno pari ampiezza, anche a Padova.

L'esempio in questione ha presentato a Padova il seguente andamento. Alle $13^{\mathrm{h}} 23^{\mathrm{m}}$, in corrispondenza di forti raffiche di vento locali, si ha improvviso aumento dell'agitazione in tutti gli apparecchi "Vicentini " della stazione (un apparecchio con periodo di ca. 2 sec. ed uno con periodo proprio di 5 sec. ca.; entrambi poco smorzati). Sull'apparecchio a breve periodo, tale agitazione ha un periodo di 2,5-2 sec.; su quello con periodo di ca. 5 sec., pure l'agitazione ha tale periodo. Essa cessa alle $13^{\mathrm{h}} 45^{\mathrm{m}}$ al cessare del vento. 


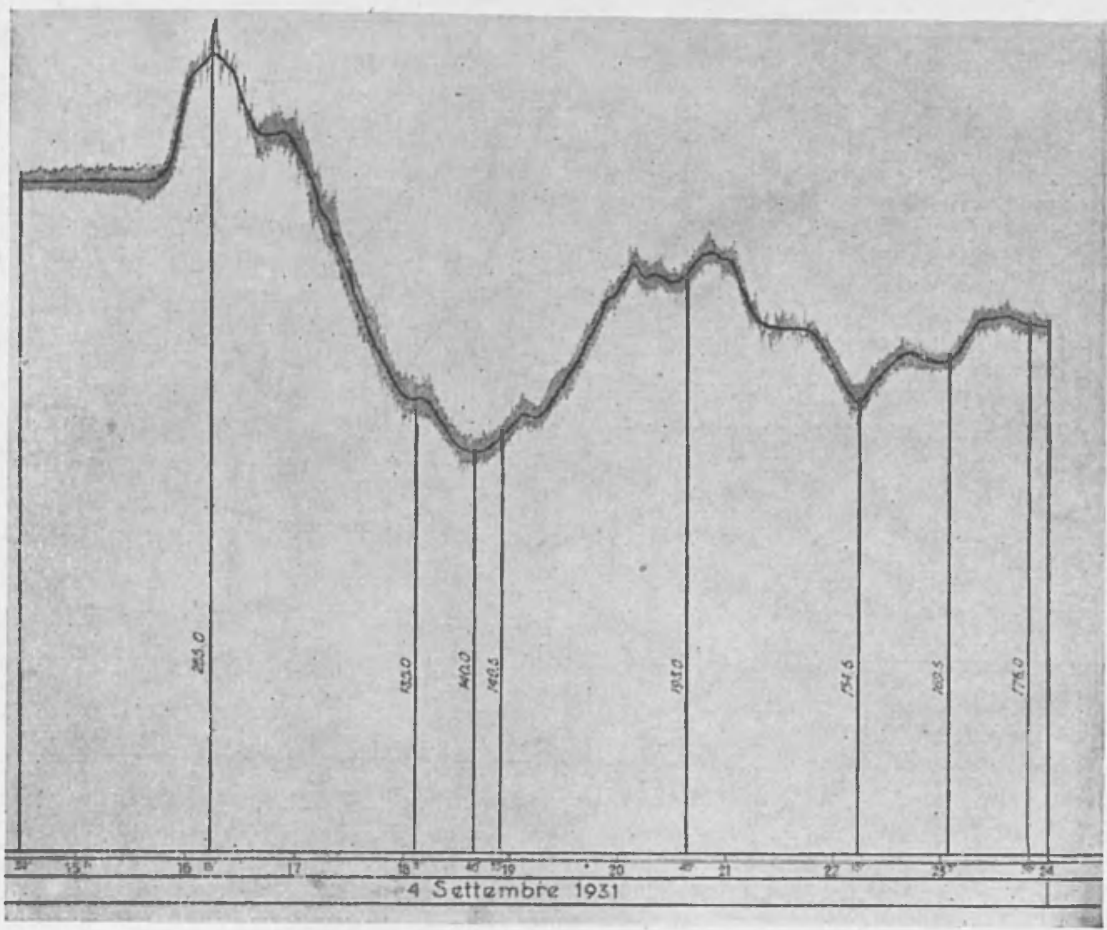

Fig. 12

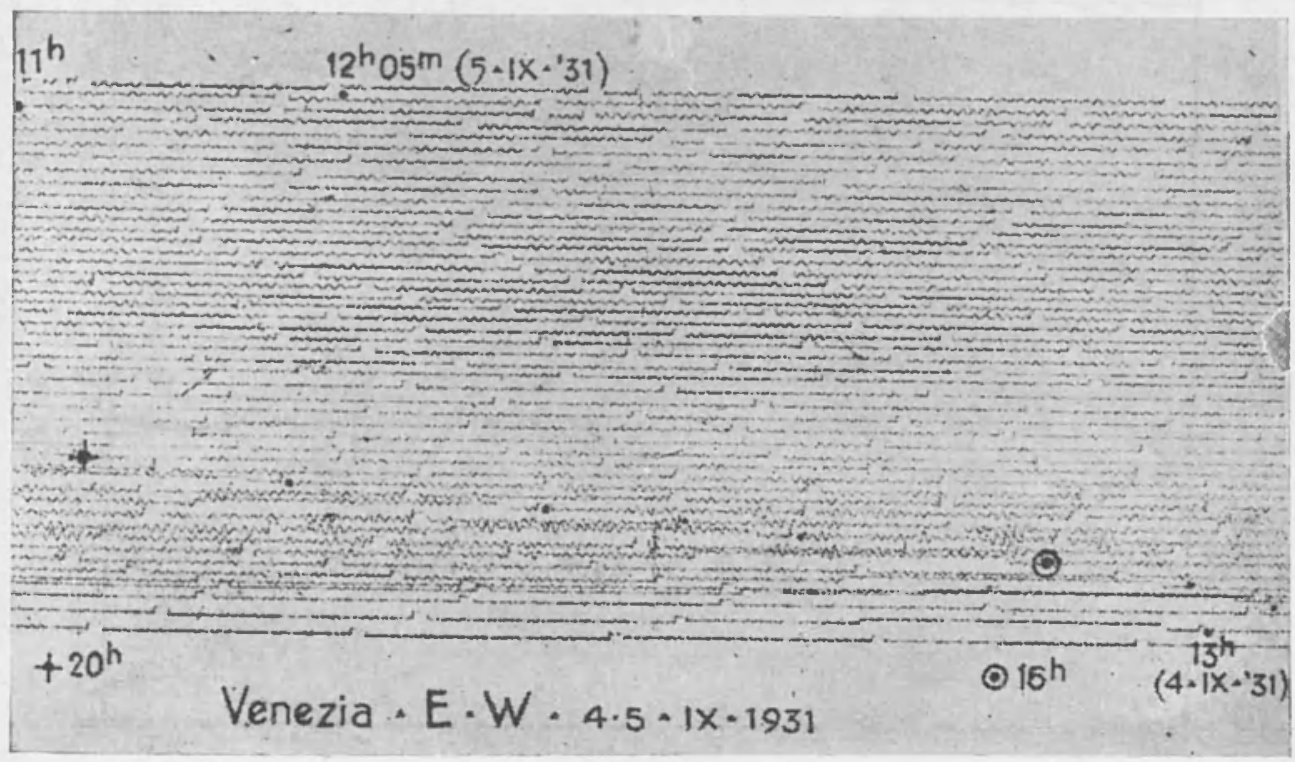

Fig. 13 


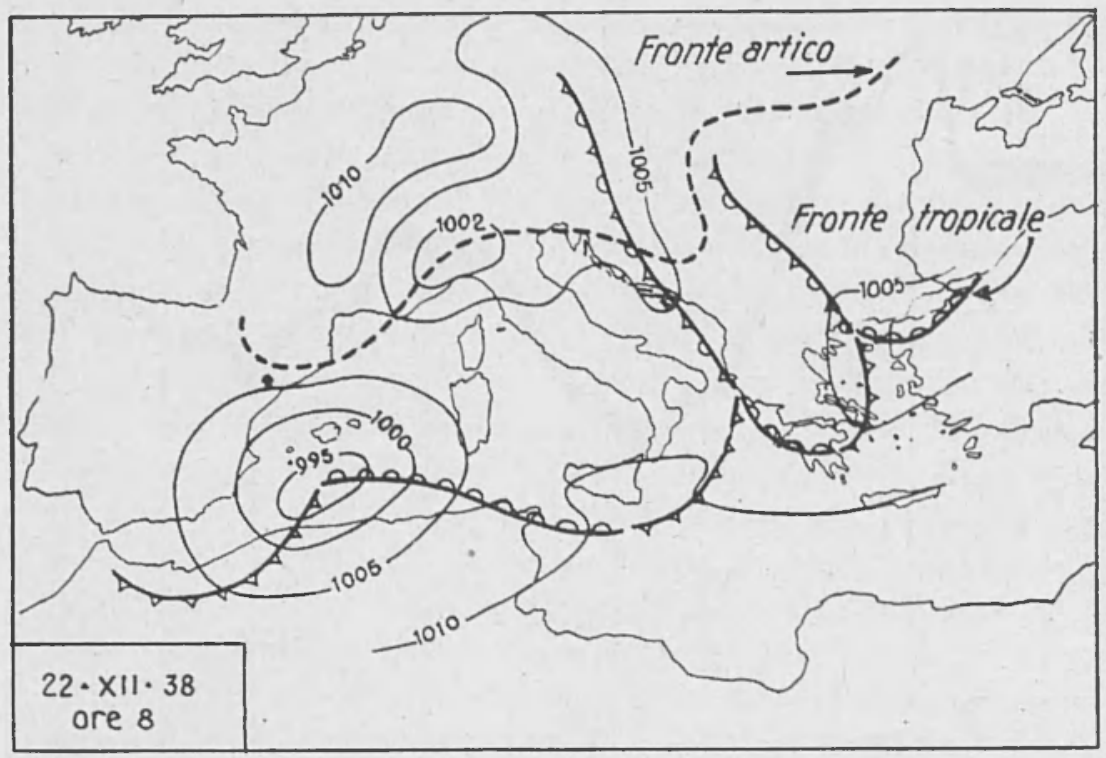

Fig. 14

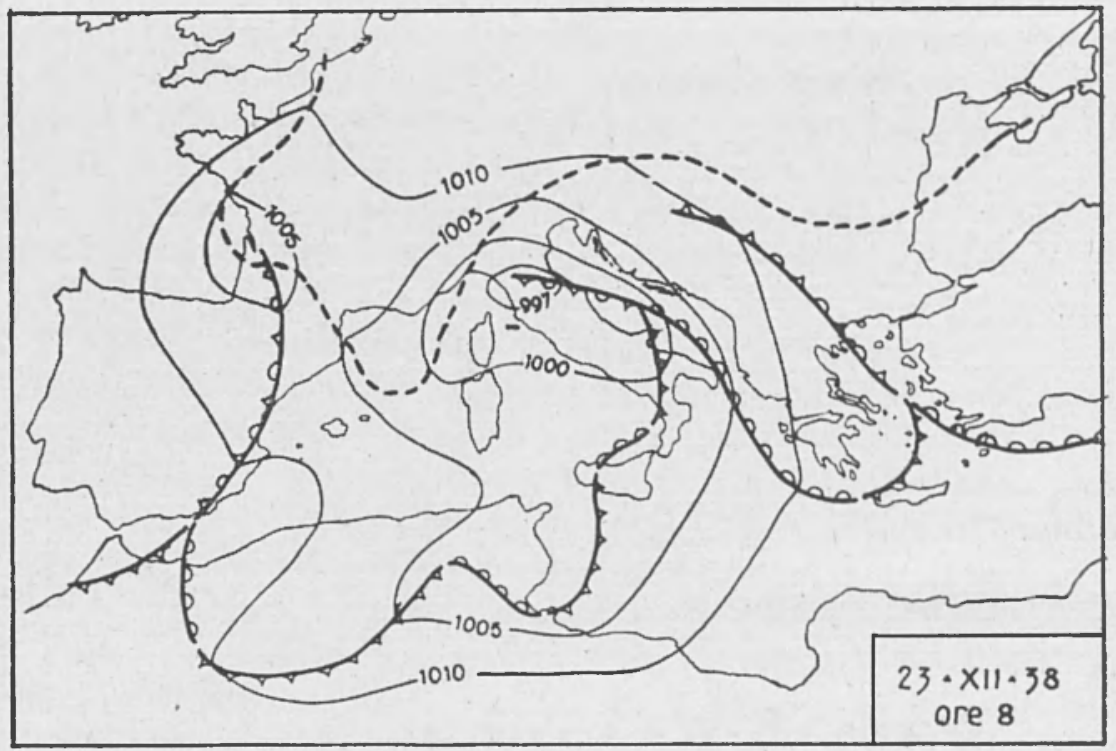

Fig. 15 


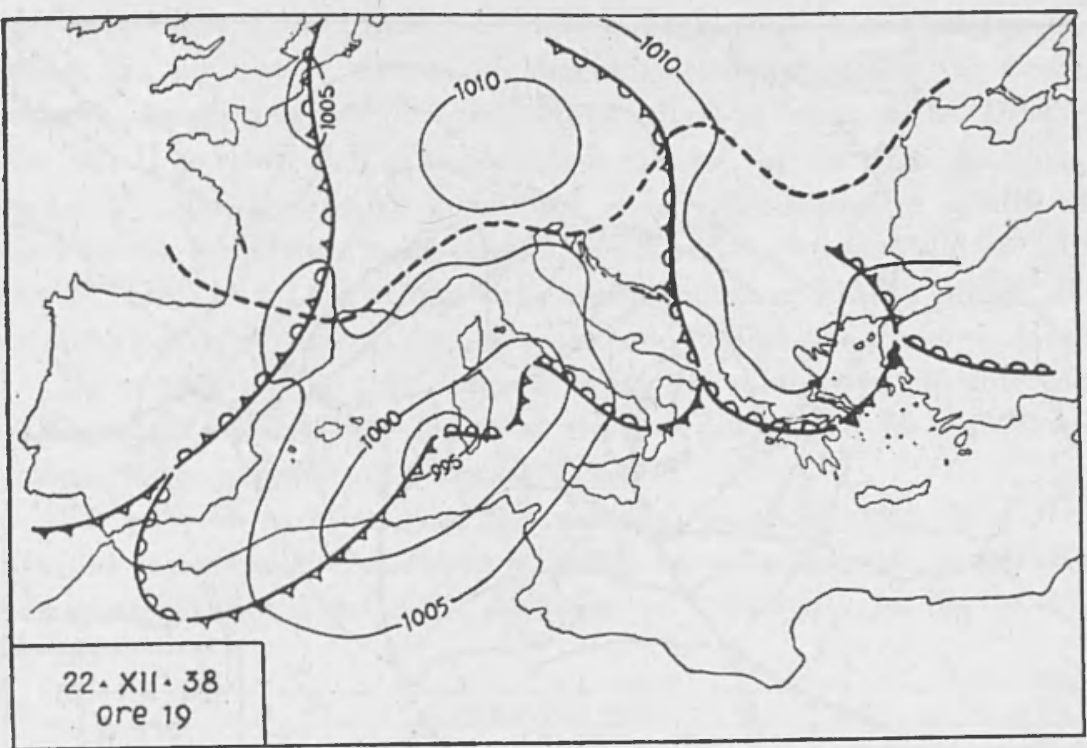

Fig. 16

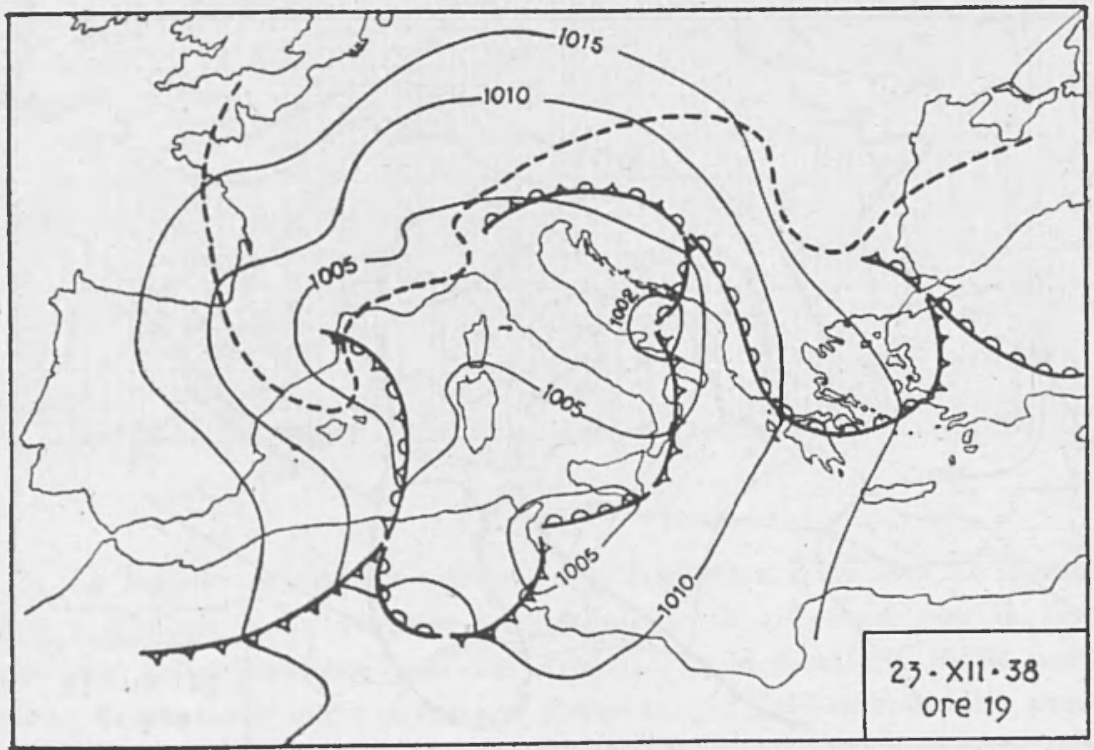

Fig. 17 


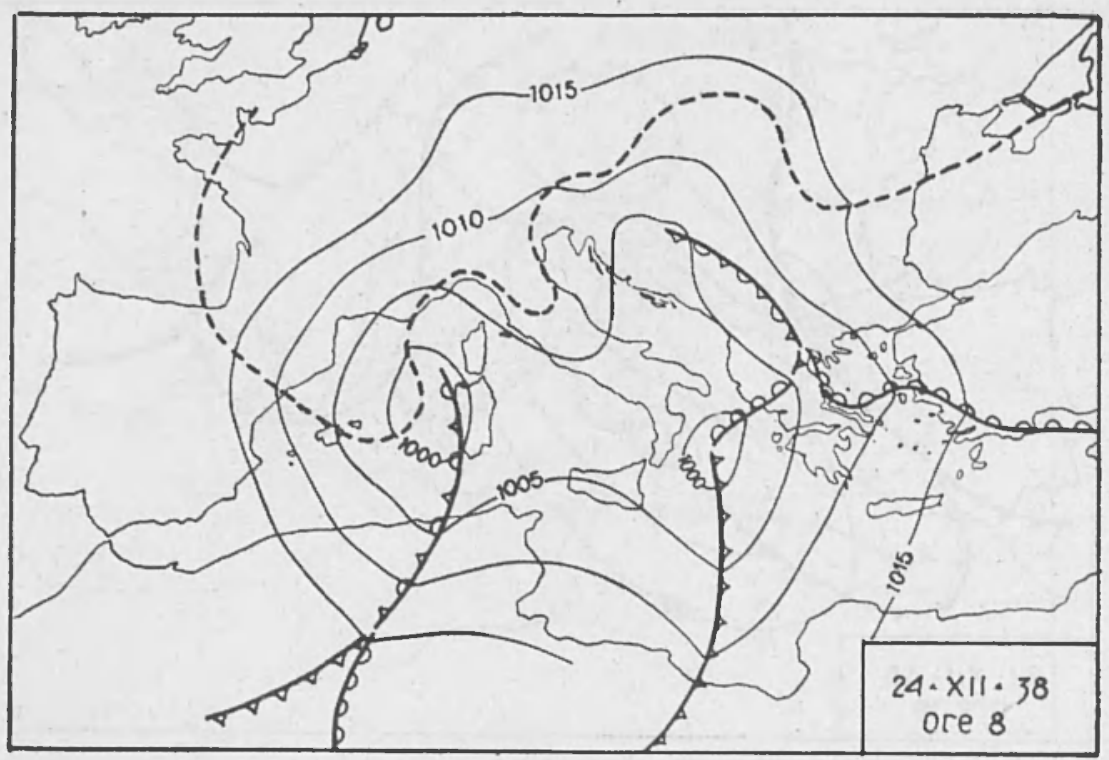

Fig. 18

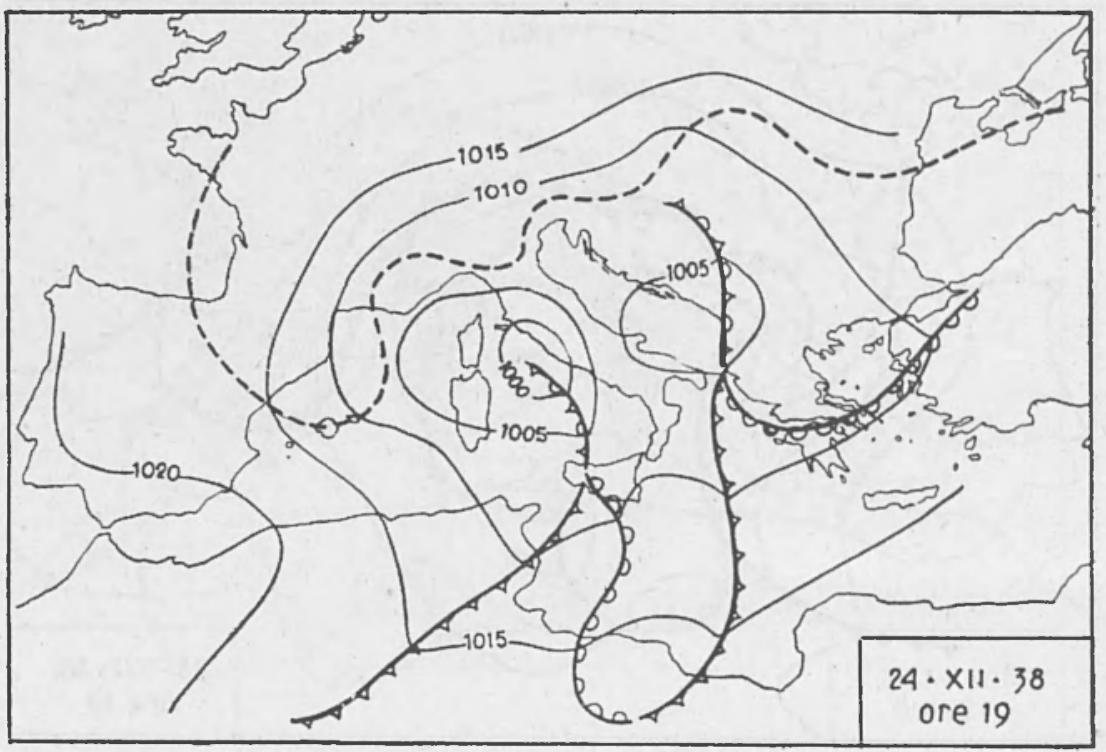

Fig. 19 
Poco dopo le $16^{\mathrm{h}}$, anche a Padova l'agitazione microsismica regolare, ha un brusco rinforzo e raggiunge, sull'apparecchio a breve periodo, ampiezze notevoli dell'ordine di $9-10 \mathrm{~mm}$ dalle $16^{\mathrm{h}} 45^{\mathrm{m}}$ alle $18^{\text {h}}$. Il periodo dell'agitazione è di $2-3$ sec. ca. su tutti gli apparecchi ("), ciò che prova come essi siano effettivamente quelli di provenienza adriatica e non, come i microsismi registrati dalle $13^{\text {h }} 23^{\text {m }}$ alle $13^{\mathrm{h}} 45^{\mathrm{m}}$, dovuti all'azione del vento, che si manifesta anche direttamente sugli strumenti, i quali reagiscono quindi con il loro periodo.

Da notare, anche qui, come la causa dei microsismi di notevole ampiezza sia strettamente legata al transito del nucleo di rapide variazioni di pressione sul golfo di Trieste.

Un esempio caratteristico della validità generale della [3] è fornito dal regime ciclonico esistente nell'Italia settentrionale e nell'alto Adriatico nei giorni dal 21 al 24 dicembre 1938 (figg. 14, 15, 16, 17, 18, 19).

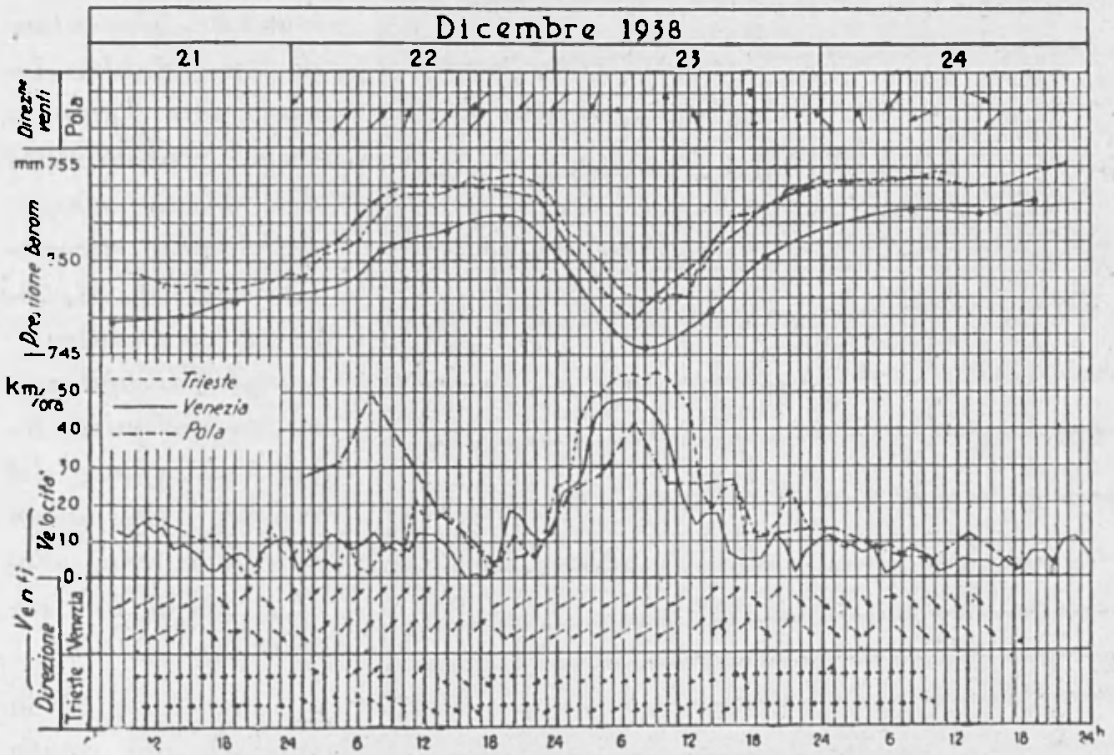

Fig. 20

La leggera agitazione microsismica registrata il 21 e il 22 dicembre a Venezia e a Trieste è probabilmente in relazione con la fase positiva della pressione sull'alto Adriatico. Col livellarsi della pressione, l'agitazione viene a cessare. Verso le $20^{1 /}$, l'abbassarsi della pressione a Venezia testimonia dell'inoltrarsi di un nucleo ciclonico nell'alto Adriatico (fig. 20). L'agitazione è sempre pressoché nulla du- 


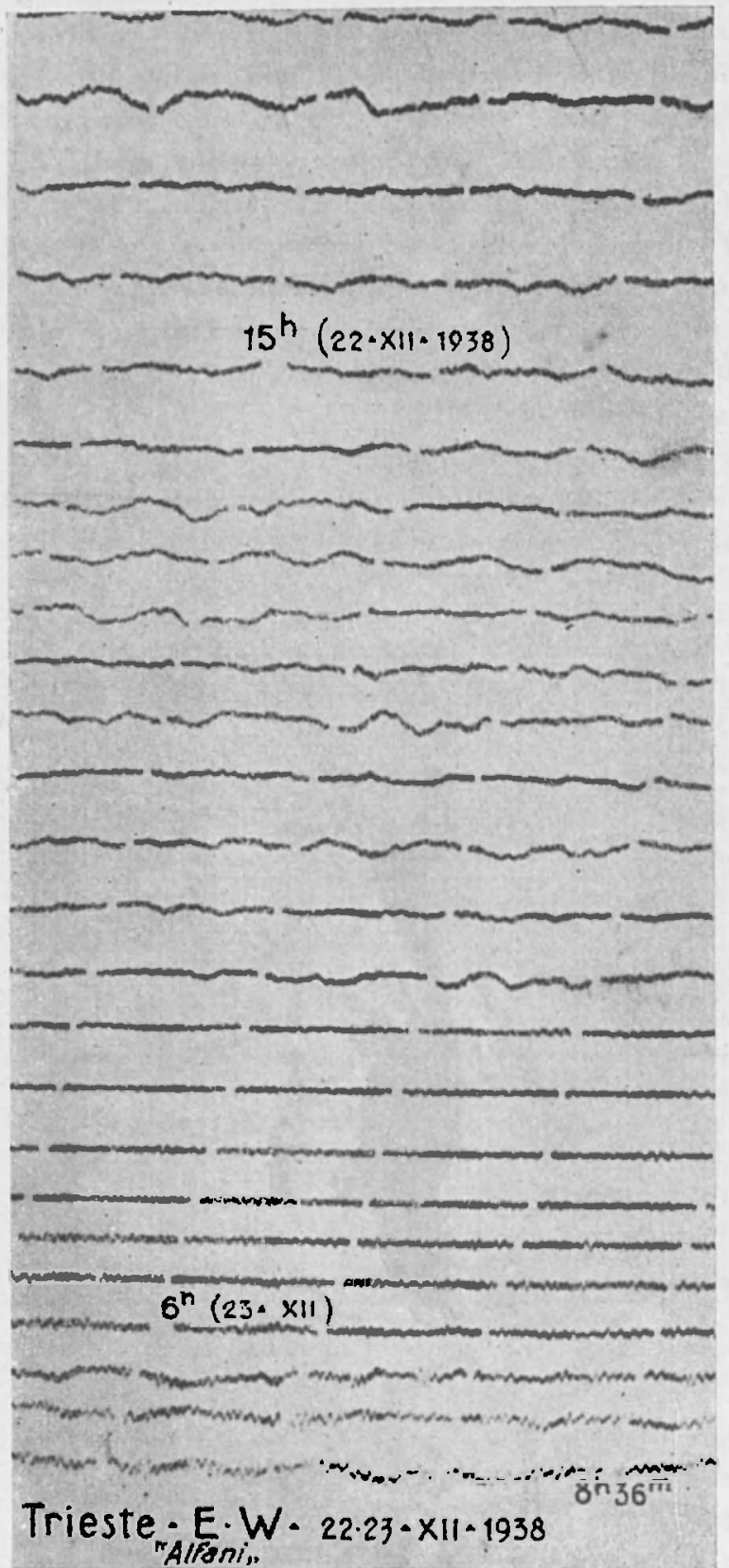

Fig. 21 rante la prima fase dii discesa del barometro: essa comincia a farsi sensiljile invece verso le $2^{\text {ln}}$, con l'insorgere e il progressivo rinforzarsi del vento di ENE a Venezia e a Trieste. La pressione continua a decrescere fino alle $8^{h}$ (a Venezia); ciò non ostante l'agitazione si fa sempre più sensibile, presentando un indubbio legame con l'aumentare dell'intensità del vento, sempre della stessa direzione (figg. 21, 22, 23, 24). Ecco un altro $e$ sempio di agitazione microsismica, le gata al soffiare del vento nella stessa direzione, con velocità pressoché costante.

Verso le $9^{\text {hi }}$ la velocità del vento comincia a diminuire e dalle $12^{\text {li }}$ alle $13^{\text {h }}$ si riduce a soli $20 \mathrm{~km} /$ ora; a quest'ora, la direzione è mutata e variabile. Malgrido ciò, sia a Trieste che a Vene- 
zia, l'agitazione microsismica cresce ancora di intensità: ciò è in stretta relazione con la ripresa della pressione caratterizzata da rapide variazioni della pressione montante in transito sullalto Adriatico, cosi da

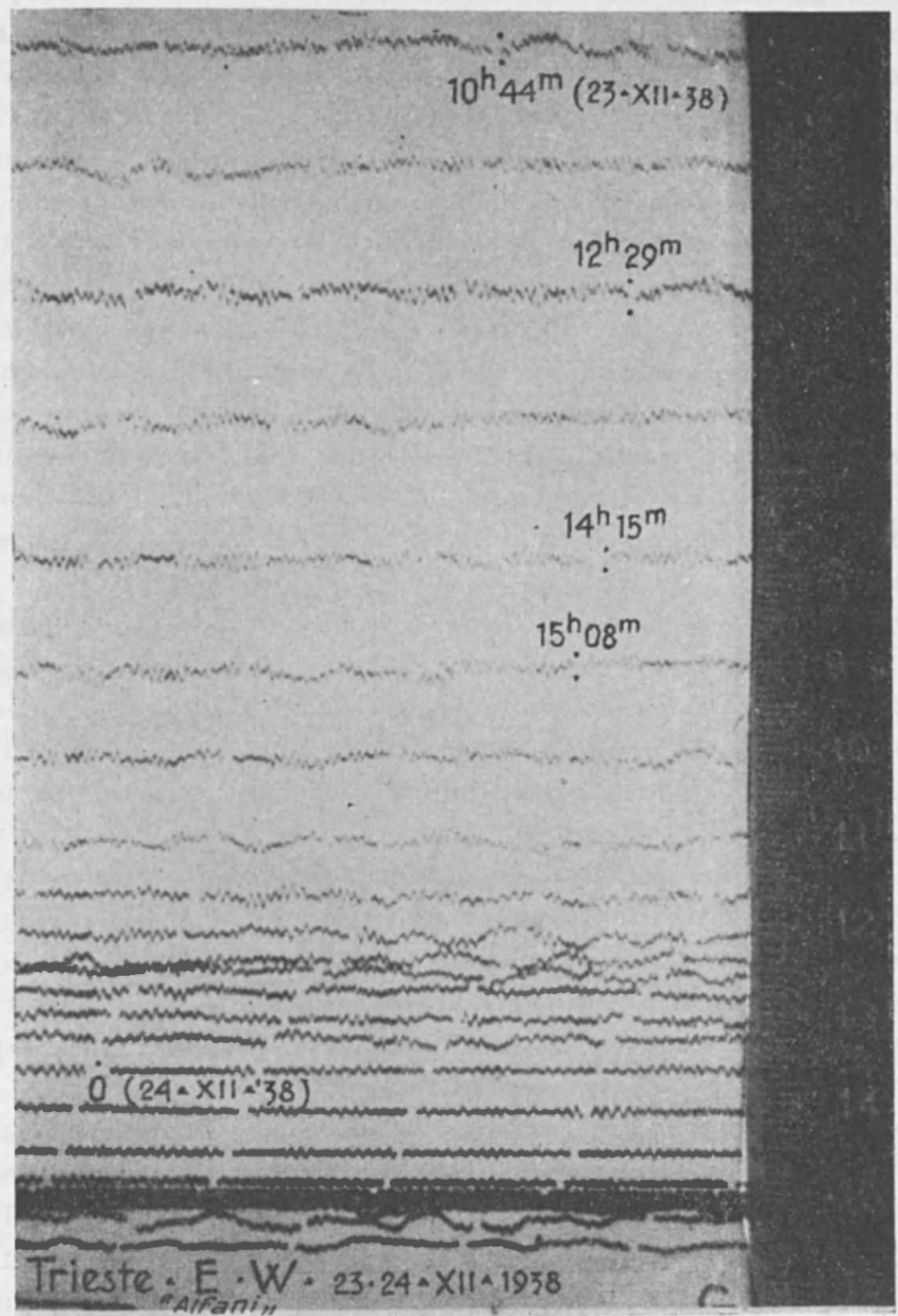

Fig. 22

soddisfare alla [3]. Verso le $29^{11}$, col livellarsi della pressione, cessa praticamente ogni agitazione microsismica (figg. 21, 22, 23, 24). Che, in questo caso, il fenomeno si spieghi più con la [3] che con la [5] 
è provato dal fatto che il ciclone è transitato a Sud del golfo di Trieste, com'è testimoniato dalla mancata formazione della sessa fondamentale del golfo.

La registrazione del 3 dicembre 1935 ha un interesse a parte. I microsismi registrati non furono ampli né a Trieste (fig. 26), né a Venezia (fig. 25). Si tratta questa volta di un ciclone di sensibile profondità, che segue il tragitto abituale W-E (fig. 27). Il fatto che i microsismi non risultino di un'ampiezza paragonabile a quella avuta nell'occasione del passaggio di cicloni, anche notevolmente meno profondi, è forse da attribuire alla velocità di transito, nel caso attuale sensibilmente maggiore a quella delle onde libere del mare sottostante.

Va qui osservato che, a differenza di quanto generalmente si verifica, in questo esempio i microsismi, sia pure di lievissima entità, cominciano a Venezia ancora prima che il centro del ciclone tocchi il mare e si fanno sensibili appena il fondo della depressione inizia il suo tragitto sul mare, per rinforzarsi via via che la depressione si avvicina all'altra sponda e cominciano ad agire $i$ nuclei di tendenza positiva.

Quindi, nel caso di depressioni particolarmente profonde, possono aversi microsismi anche al di fuori del verificarsi della condizione $\mathrm{V} / \mathrm{c}=-1$ nella [5], pure se il centro del ciclone non ha ancora raggiunto il mare. In questo caso però, i microsismi appaiono di lieve entità, e riescono appena pércettibili se il ciclone è ancora su terra ferma.

Non sempre i nuclei di perturbazione in transito sull'alto Adriatico, pur seguendo il tragitto da Ovest ad Est in modo da soddisfare approssimativamente alla condizione $V / c=-1$ nella [5], generano microsismi apprezzabili. Ciò dipende dal fatto che non in tutti i nuclei perturbanti esistono variazioni rapide di pressione, tali da perturbare il mare così da causare microsismi.

Ecco un esempio. Il 26 settembre 1936 transita sull'alto Adriatico un'onda di pressione con periodo di $3^{\text {h }}$ ca. (fig. 32). Dopo aver toccato Venezia alle $15^{\text {hl }}$ ca., detta oscillazione perviene a Trieste alle $18^{11}, 4$ ca., avendo attraversato l'alto Adriatico alla velocità di $48 \mathrm{~km} /$ ora ca. $\mathrm{Nel}$ golfo di Trieste quest'onda determina la formazione di un'ampia sessa fondamentale (fig. 32), la direzione e la velocità di propagazione essendo state conformi alla relazione $V / c=-1$. Come provano i barogrammi ottenuti a Venezia e a Trieste, l'onda barometrica accennata appare senza accidentalità, ciò che prova dell'esiguità delle rapide variazioni di pressione sovrapposte. Tali variazioni però non do- 
vevano esser del tutto mancanti. Infatti, col sopraggiungere della perturbazione sul golfo di Trieste, una minutissima agitazione microsismica viene registrata, contemporaneamente, a Trieste e a Venezia

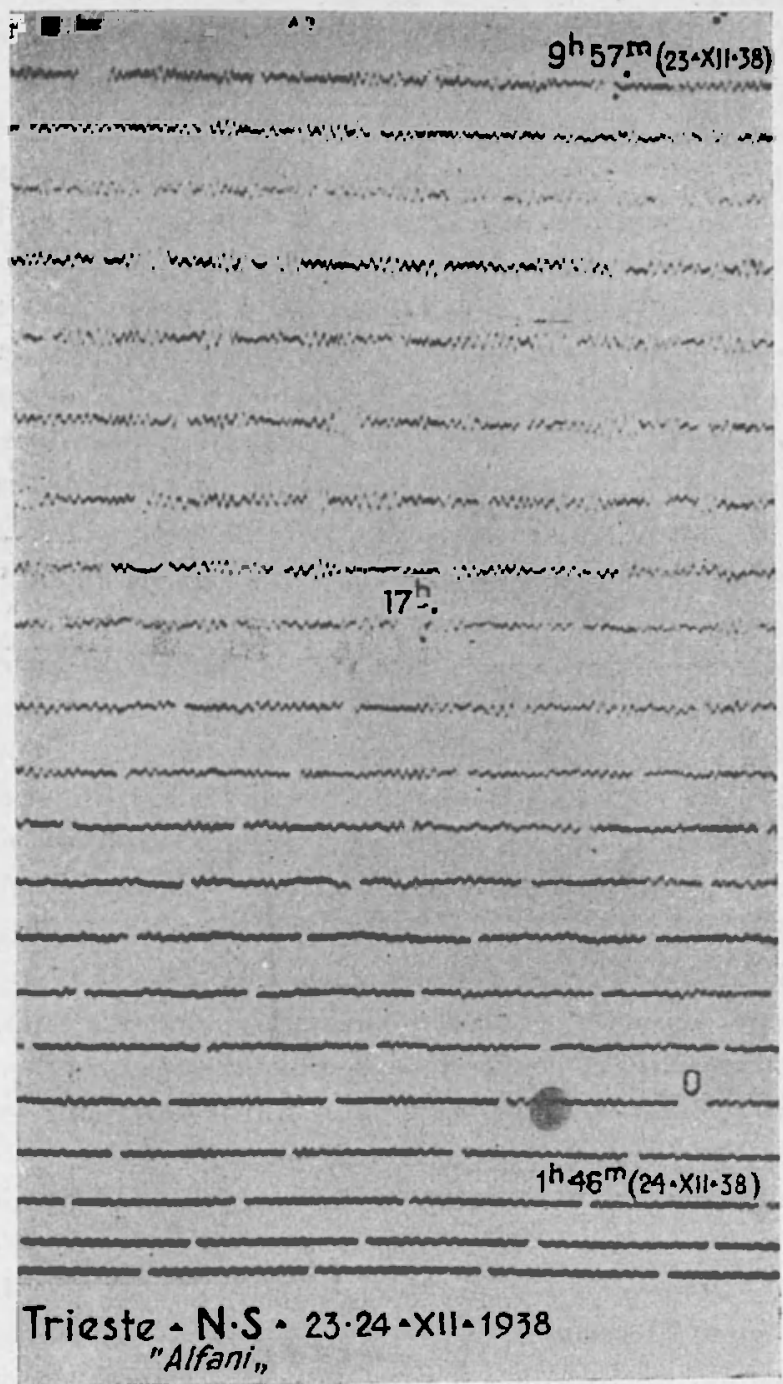

Fig. 23

(figg. 33, 34, 35 - oscillazioni regolari, rapidissime dalle $18^{\text {h }}$ alle $19^{\mathrm{h}}$ ca), ciò che costituisce un'ulteriore testimonianza della validità della [5], nel caso $V / c=-1$, anche per rapide perturbazioni di pic- 
colissima entità. $\mathrm{E}$ interessante osservare che tale minutissima agitazione ha un periodo di 1,5 sec. circa. Vedremo più oltre come può essere spiegata la variazione del periodo per $i$ microsismi interessanti l'alto Adriatico.

L'esempio del 26-27 novembre 1933, è interessante perché fornisce, separati, microsismi dovuti a bora e al transito di rapidi disturbi microbarici - legati a pressione in fase montante - obbedienti alla relazione $V / C=-1$. Come risulta dalla fig. 28 - che sintetizza la situazione meteorologica a Trieste e a Venezia - e dalle figg. 29, 30 e 31 (nella quale ultima va letto 1933 in luogo di 1931), i microsismi registrati a Venezia e a Trieste dalle 12 alle 24 sono causati dall'azione della bora sul mare e, nel caso di Trieste, anche sugli edifici (caratteristiche per Trieste le perturbazioni irregolari, dovute all'azione diretta); dall' $1^{\mathrm{h}}$ alle $11^{\mathrm{h}}$ del 27 quando il vento era pressocho cessato, insorgono i microsismi dovuti all'azione dei disturbi microbarici, agenti prima sul mare, quindi, direttamente, sul fondo: microsismi regolari, a breve periodo e a sensibile componente verticale.

2. - Generalmente si parla di cause dei microsismi, prescindendo dalle condizioni in cui queste cause agiscono. Questa impostazione del problema è incompleta e può condurre, come ha condotto, a conclusioni soltanto parziali o addirittura fallaci. A mio parere, l'altro elemento sostanziale, che va considerato nel problema dei microsismi, è quello che si riferisce al mezzo e alle sue caratteristiche fisiche. Coloro che ritengono causa esclusiva dei microsismi l'azione delle oscillazioni microbariche, di origine ciclonica, dimenticano che vaste zone del fondo marino si mostrano insensibili a detta azione. D'altra parte, i sostenitori della teoria dei marosi sembrano ignorare che estesissime zone costiere - e non tutte con coste pianeggianti - non vengono affatto eccitate dall'urto delle onde marine.

Mi limito, a questo riguardo, a due esempi estremamente significativi.

a) Abbiamo visto che durante il transito delle due formazioni temporalesche del 13 ottobre 1933 e del 16 ottobre 1934 sull'alto Adriatico, l'agitazione microsismica ha assunto bruscamente ampiezze sensibili col pervenire del nucleo ciclonico sul golfo di Trieste.

La fig. 36 dà la rappresentazione schematica, in sezione, dell'alto Adriatico, tra la località Cavallino (Venezia) - A e Barcola (Trieste) - B. Tale rappresentazione è stata tracciata da G. Andreotti, sulla base di accurate ricognizioni geologiche $\left({ }^{6}\right)$. 


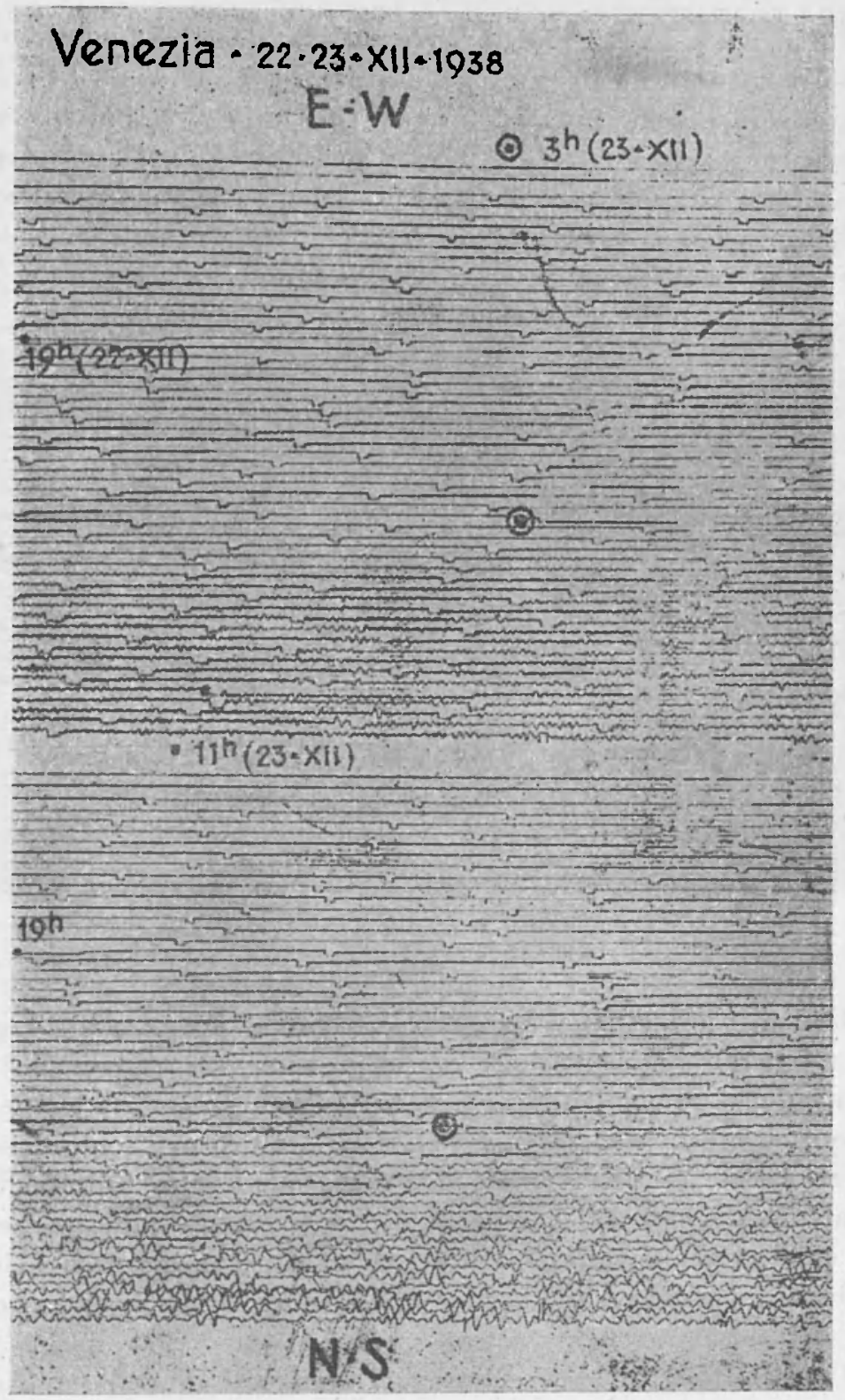

Fig. 24

Lo strato d'acqua non supera mai i $20-25 \mathrm{~m}$. Segue uno strato alluvionale (AI) quaternario, costituente il letto del mare ed avente uno spessore da 300 a $400 \mathrm{~m}$; ad esso segue lo strato pliocenico P1 
di sabbie fini e argille, spesso circa $200 \mathrm{~m}$; lo strato miocenico $M$ sottostante, costituito da arenarie, per uno spessore di $200 \mathrm{~m}$ circa, è seguito da marne e calcari formanti lo strato oligocenico-eocenico (Al-Eo) con spessore variabile fra 250 e $350 \mathrm{~m}$. Il calcare compatto del Cretaceo $C r$, comincerebbe quindi ad una profondità dell'ordine di $1000 \mathrm{~m}$. Questo per quanto si riferisce alla parte centrale della regione Ven.-Trieste. E da osservare che il sottosuolo della bassa Valle Padana, in generale, ba analoga struttura sedimentaria.

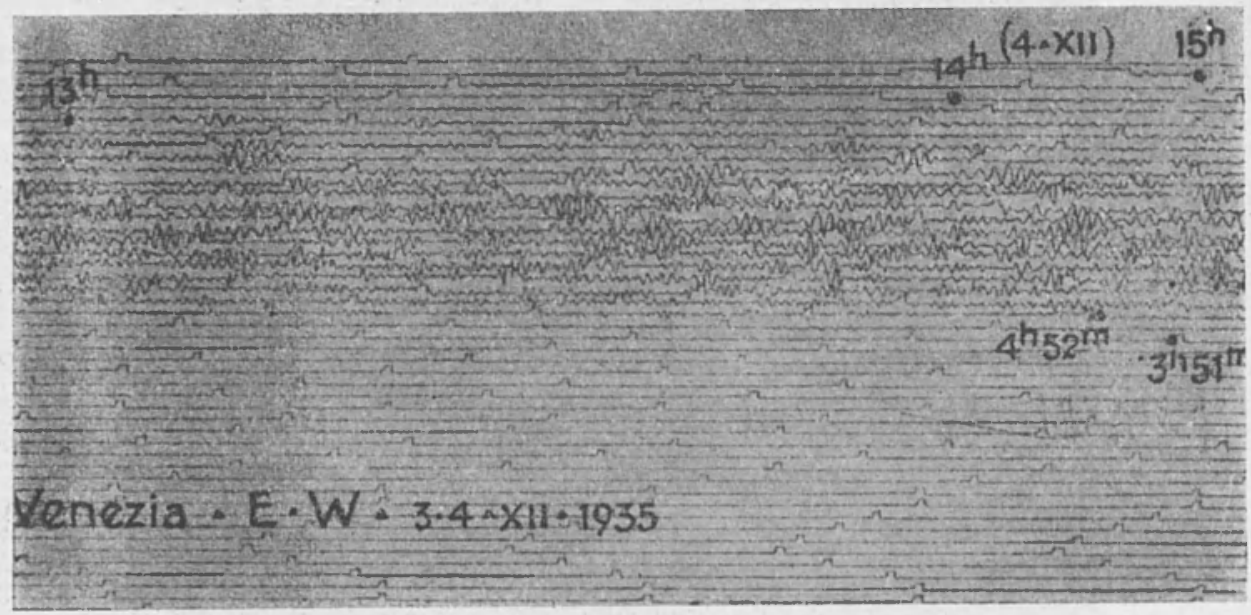

Fig. 25

Dall'apertura esterna del golfo di Trieste, procedendo verso la base, gli strati sedimentari su considerati vanno assottigliandosi sia in senso longitudinale ehe trasversale. Va ancora osservato cbe le formazioni cicloniche nelle regioni dell'alto Adriatico non sono mai - o solo raramente - vaste e profonde come quelle cbe interessano gli oceani o le regioni dell'Europa settentrionale: sono quindi da escludere quelle violente variazioni di pressione che si verificano nei cicloni nordici, né il moto ondoso assume mai le proporzioni gigantesche dei mari del nord.

Ciò può forse spiegare, insieme al verificarsi di altre condizioni, come proprio nel ristretto bacino del golfo di Trieste il ciclone in transito eserciti la sua massima azione sul fondo, azione che è indubbiamente legata anche alla velocità di transito dei disturbi microbarici e che diviene massima quando detti disturbi hanno velocità paragonabile a quella delle onde libere del mare sottostante. 


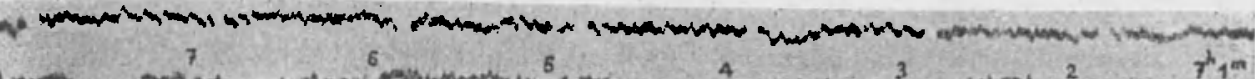

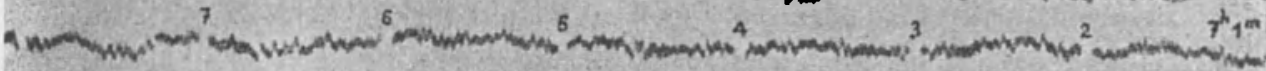

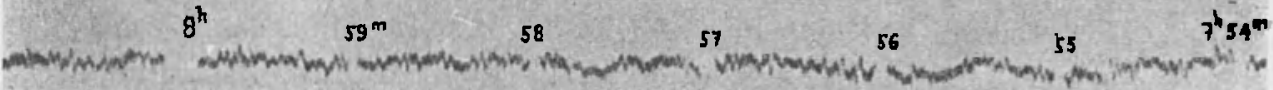
52
51.
50
$4 \overline{9}$
48
$8^{\mathrm{b}}+47^{m}$

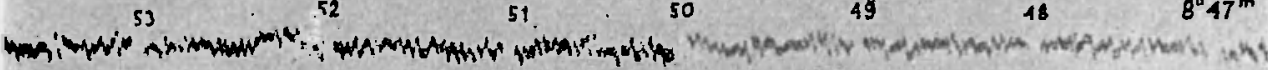

\section{Trieste $\cdot 3 \cdot 4 \cdot \times 11 \cdot 1935$}

$$
41 \quad 9^{2} 40^{m}
$$

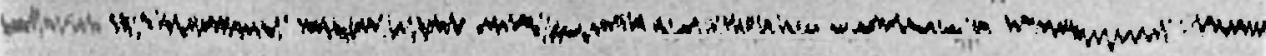

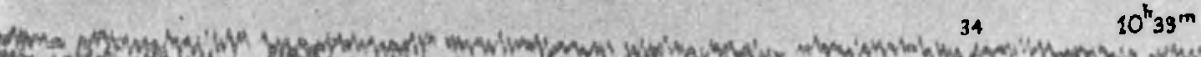

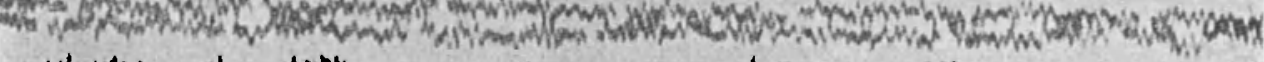

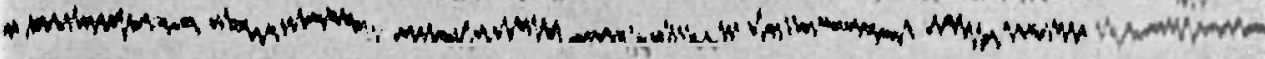

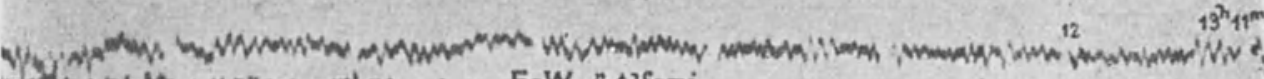

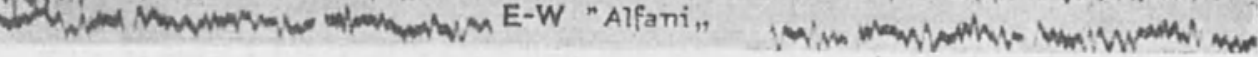

Fig. 26

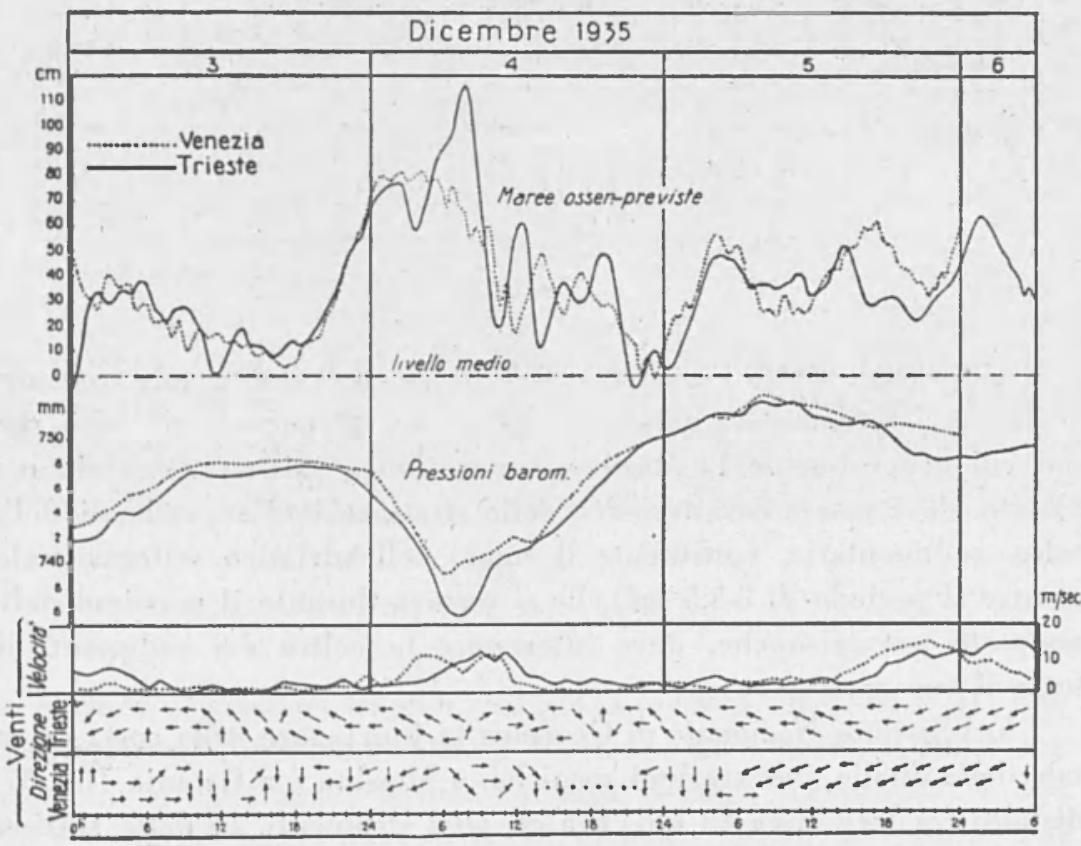

Fig. 27 
L'agitazione originata nel golfo di Trieste viene esaltata a Venezia e a Padova, dove fornisce registrazioni più vistose che non a Trieste, non ostante la maggiore distanza. Ciò sta a provare che la via di propagazione dell'agitazione microsismica è costituita dagli strati sedimentari sopra citati : è noto infatti che gli strati alluvionali, quando sono di potenza limitata, esaltano le sollecitazioni sismiche che in essi si manifestano.

Qui viene opportuna un'altra osservazione riguardante il periodo

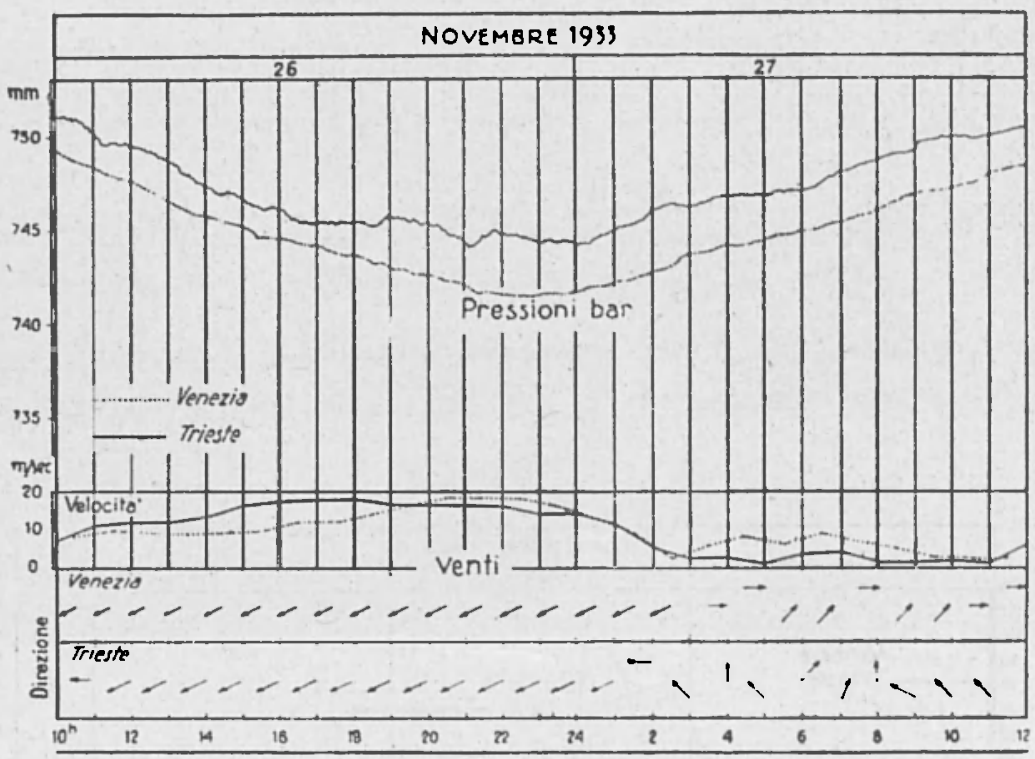

Fig. 28

dei microsismi. Detto periodo è indubbiamente legato allo spessore degli strati sedimentari, messi in agitazione. Il periodo di 1-1,5 sec, con cui generalmente l'agitazione microsismica inizia a Venezia e a Trieste, deve essere caratteristico delle stratificazioni superficiali della coltre sedimentaria, costituente il fondo dell'Adriatico settentrionale, mentre il periodo di 3-3,5 sec, che si osserva durante il massimo delle tempeste microsismiche, deve interessare la coltre dei sedimenti in tutto il suo spessore.

b) L'Istituto Nazionale di Geofisica fa funzionare sulla costa orientale della Sicilia, due stazioni sismiche, a Messina e a Catania (fig. 37), distanti fra loro circa $80 \mathrm{~km}$. Fra gli altri strumenti, in dette stazioni funzionano due coppie di sismografi tipo Wiechert, aventi costanti 
strumentali identiche. Il 24, 25, 26, 27 gennaio 1950 tutto il mar Jonio e Io stretto di Messina furono sconvolti da vento ciclonico (figg. da 38 a 45). A Catania, come al solito in analoghe occasioni, si ebbe la registrazione di microsismi di eccezionale ampiezza dell'ordine di $70 \mu$,

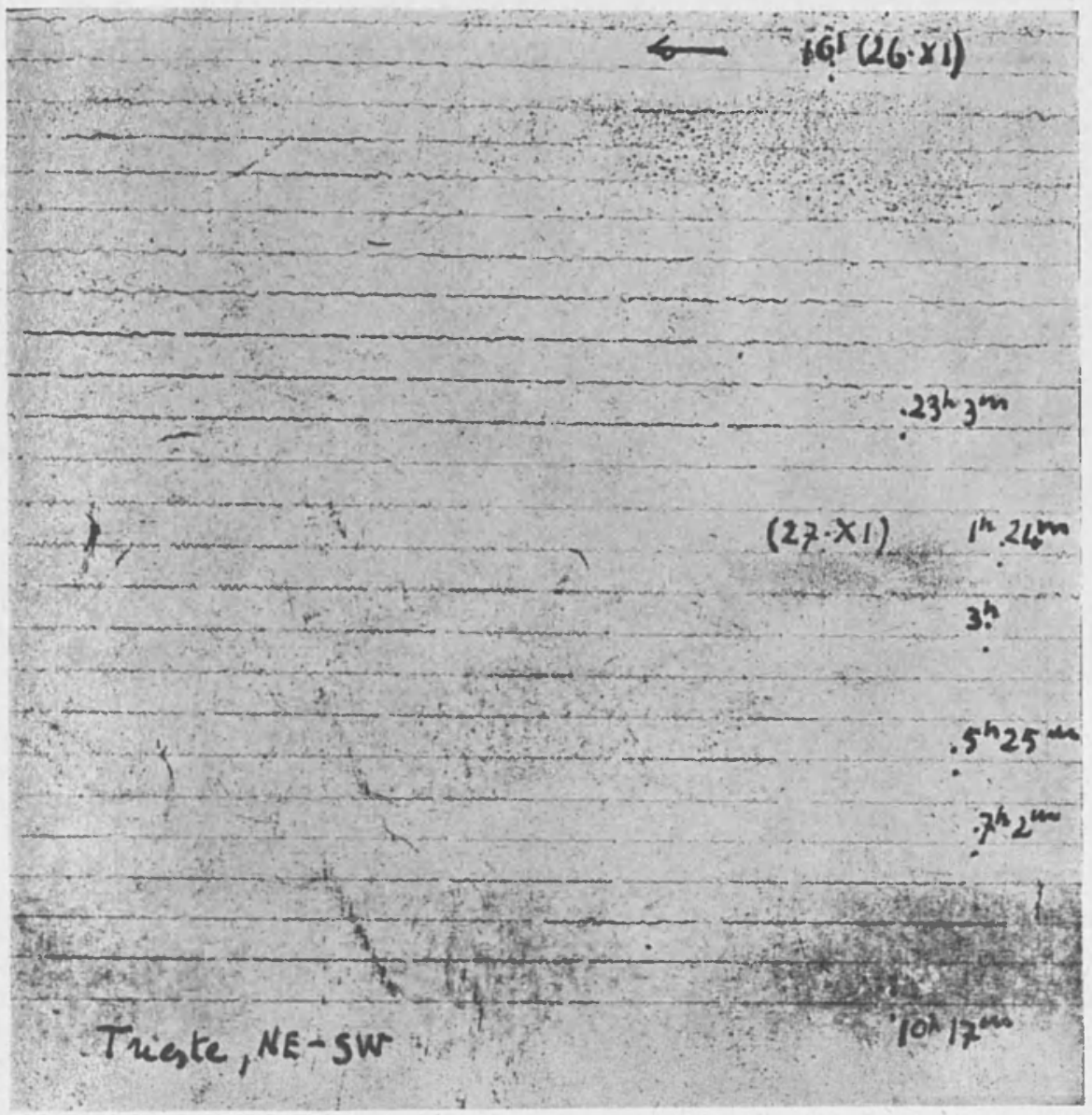

Fig. 29

con periodi di 2,5-3,5-4 secondi, mentre a Messina (figg. 46 e 47) non venne registrata agitazione alcuna $\left({ }^{7}\right)$. Come si è detto, il mare era fortemente e ugualmente agitato in tutta la zona interessante le due città e i marosi battevano con ugual violenza contro le coste, il cui andamento verso il mare presenta inclinazioni comparabili. 
Ecco un esempio in cui la natura del sottosuolo ha un'influenza decisiva. Le coste e il fondo marino della zona di Catania consistono infatti di uno spesso strato di lava, mentre le coste e il fondo marino interessante Messina sono rocciose od hanno una sottile copertura di sedimenti. Qui è da mettere in evidenza un altro aspetto interessante

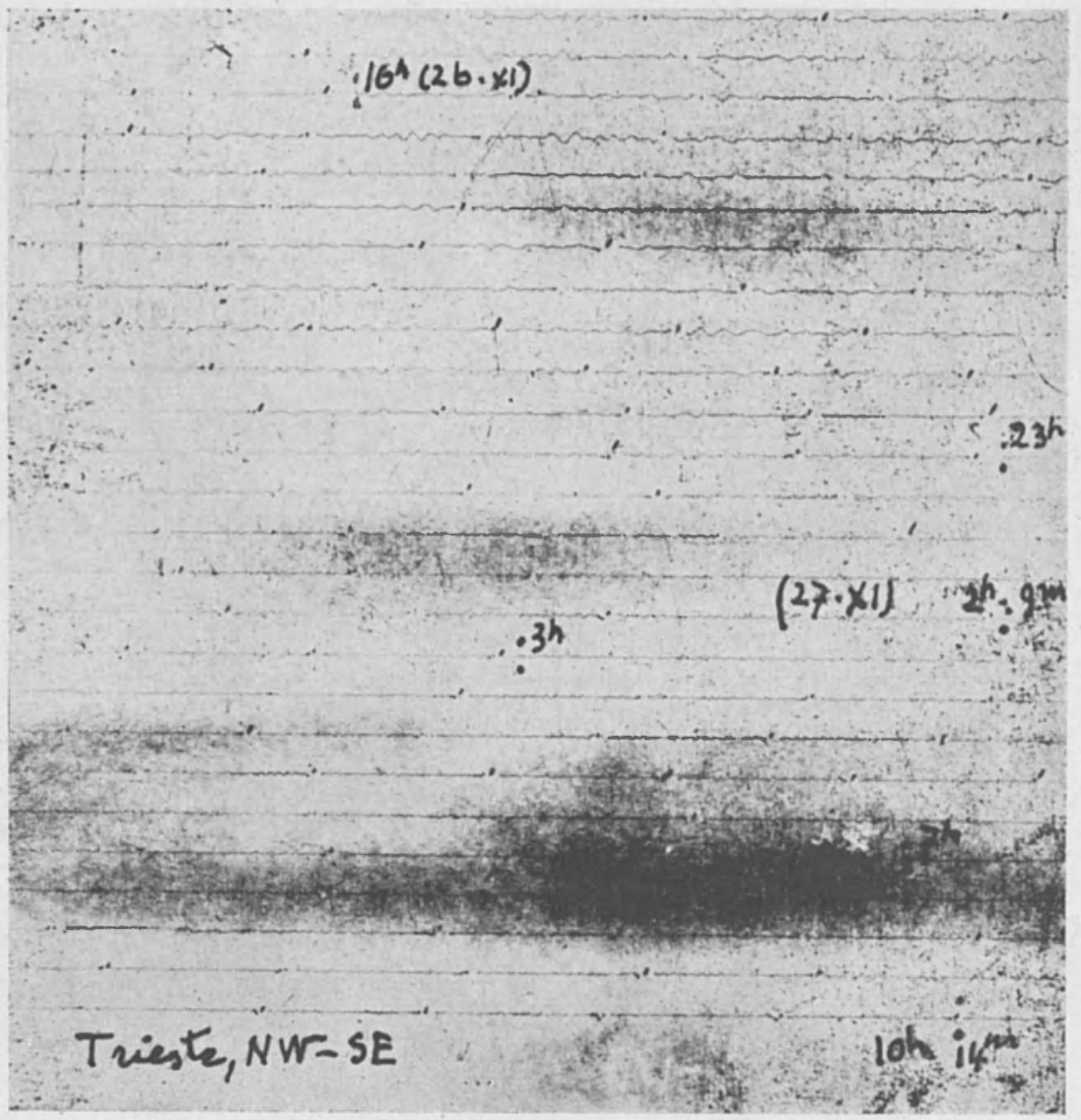

Fig. 30

della questione. Non solo certe zone sono sorde alle cause che altrove determinano microsismi, ma neppure trasmettono microsismi - anche di notevole ampiezza - generati in altre aree, pure se vicine, come nel caso appunto di Messina. Questa città dista soli $80 \mathrm{~km}$ da Catania, con linea costiera senza soluzioni di continuità : ciò non ostante, e malgrado l'eccezionale ampiezza dei microsismi registrati a Catania, l'agitazione si estingue completamente prima di giungere a Messina. 
Possono quindi sussistere delle zone di fortissima agitazione microsismica completamente isolate, in cui determinano il massimo effetto quelle cause che in zone, anche limitrofe, riescono inattive.

Nella generazione dei microsismi non è quindi tanto l'energia in giuoco la causa determinante, quanto il modo con cui tale energia

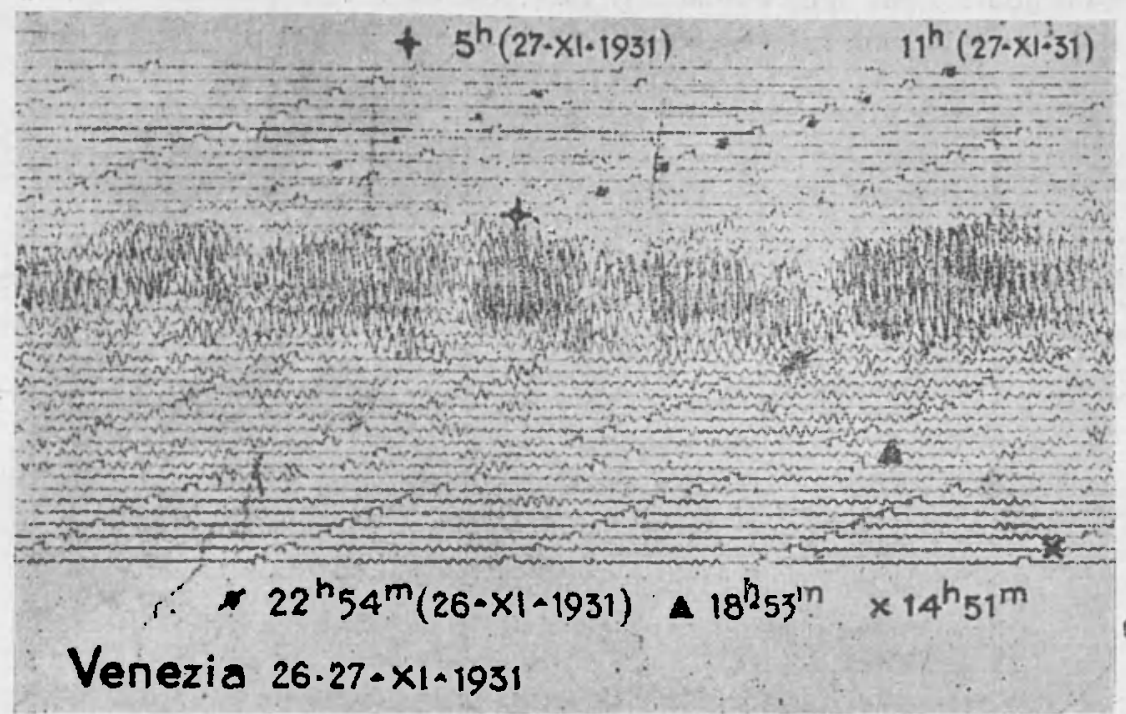

Fig. 31

si manifesta. Non è quindi necessario ricercare qual è il veicolo che conduce sul fondo del mare la massima possibile quantità d'energia.

L'energia veramente efficiente, ai fini della creazione dei microsismi, è quella legata alle oscillazioni marine di determinato periodo e di determinata velocità, che riescono ad eccitare gli strati del fondo sottostante. $\mathrm{E}$ in questa direzione che le ricerche vanno orientate, se si vuole chiarire completamente il problema.

L'eccitazione degli strati sottostanti è da ritenersi possibile solo quando l'energia, indipendentemente dalla sua entità, sarà associata a quei determinati periodi e a quelle determinate velocità.

Quando l'energia associata ai disturbi microbarici (o al vento) viene trasmessa - attraverso l'acqua che fa da mezzo di collegamento - per fenomeno di filtrazione energetica sul fondo, fate che questi sia costituito da stratificazioni capaci di risonanza con l'energia oscillante incidente e avrete la creazione dei microsismi. Se il fondo con- 
siste di stratificazioni sorde a queste sollecitazioni, il mezzo solido non diventerà fonte di perturbazione alcuna.

Solo cosi si spiega come il fondo dell'alto Adriatico possa venire eccitato dalle sollecitazioni che gli vengono dall'aria, attraverso il mare, e trasmetta le sue vibrazioni verso occidente, dove esso viene continuato nella Val Padana. E solo così ancora si può dar ragione del fatto che non solo il fondo del mare nello stretto di Messina non

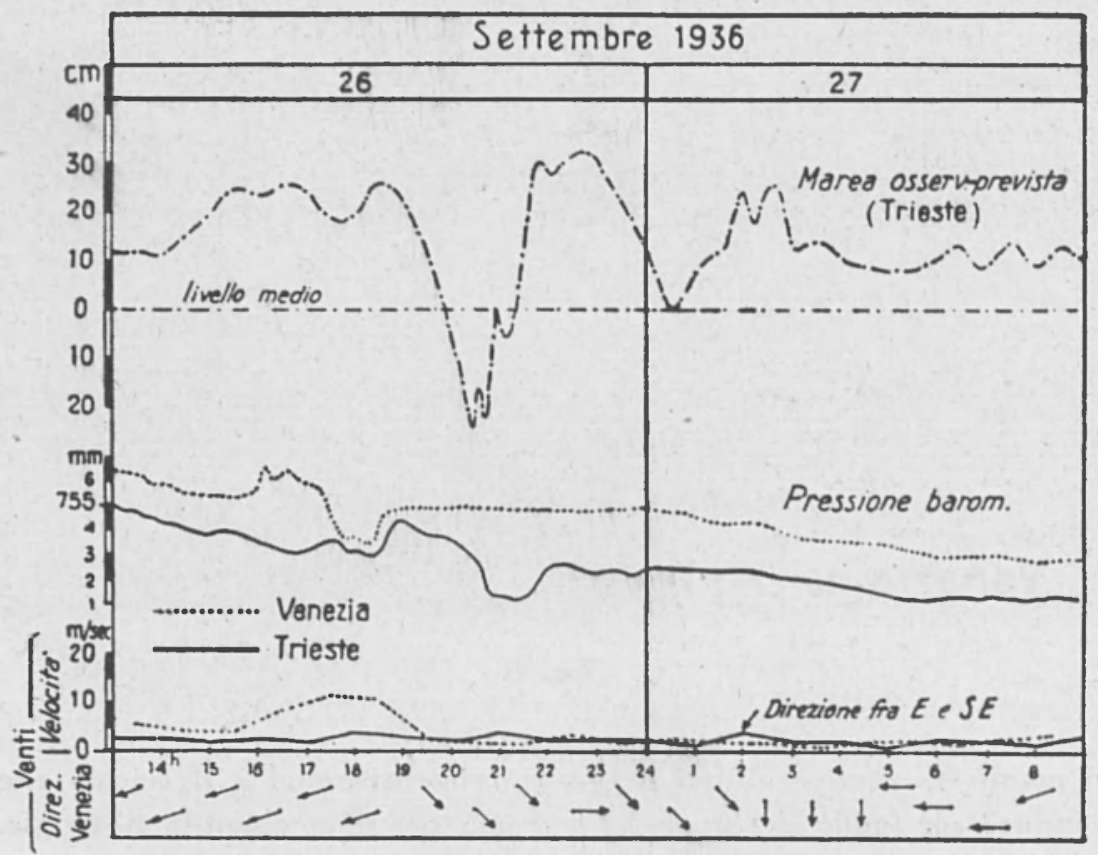

Fig. 32

entra in agitazione per sollecitazioni, talvolta amplissime, che il fondo del mare un po' più a sud riceve dal moto ondoso. Gli è che mentre, all'altezza dell'Etna, il fondo del mare è costituito da stratificazioni con gamme di oscillazioni analoghe a quelle del mare agitato, il fondo dello stretto, all'altezza di Messina, si presenta roccioso, le forti correnti marine avendo impedito la sedimentazione.

Alcuni geologi (fra i quali Cortese, Hobbs e, più recentemente, Beneo) ritengono che per lo stretto di Messina passi una grande frattura che dall'Etna va a punta Alice sullo Jonio; tale frattura taglierebbe, con un piano inclinato verso $\mathrm{E}$, il terreno cristallino che, secondo Cortese, forma direttamente il fondo dello Stretto, con una 


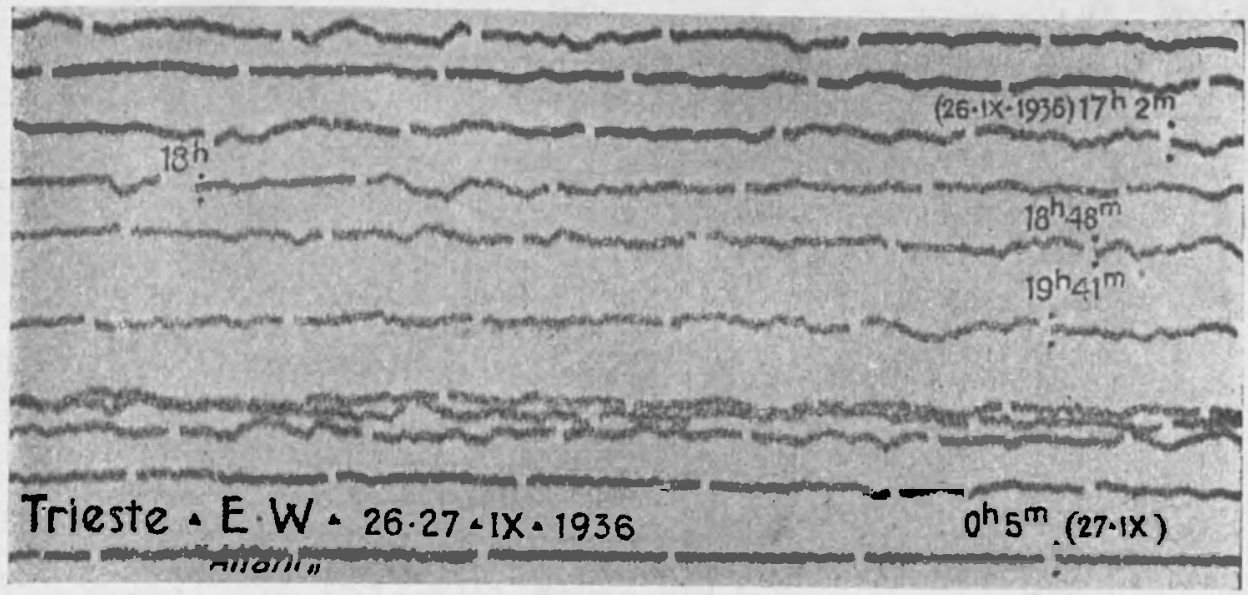

Fig. 33

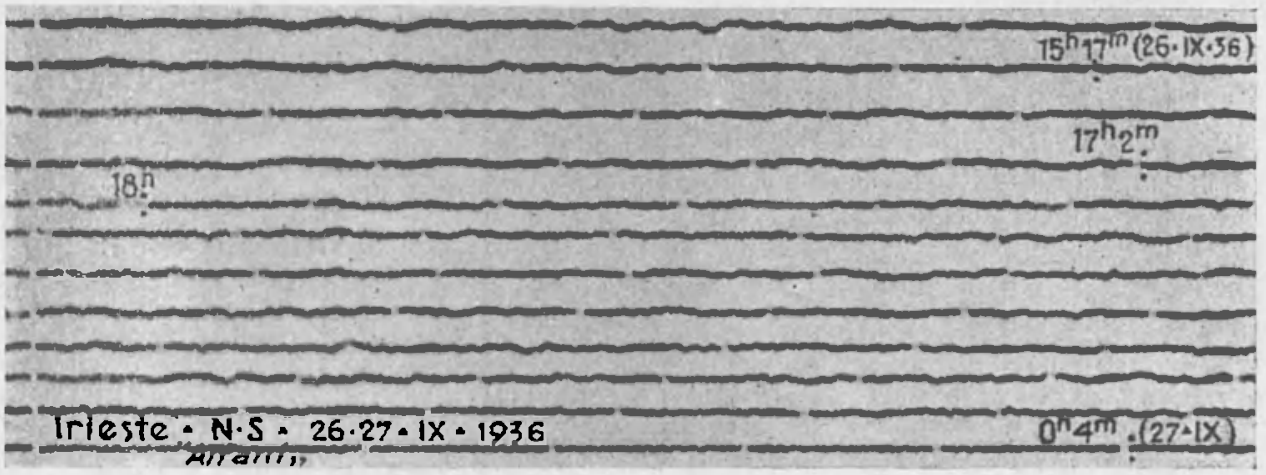

Fig. 34

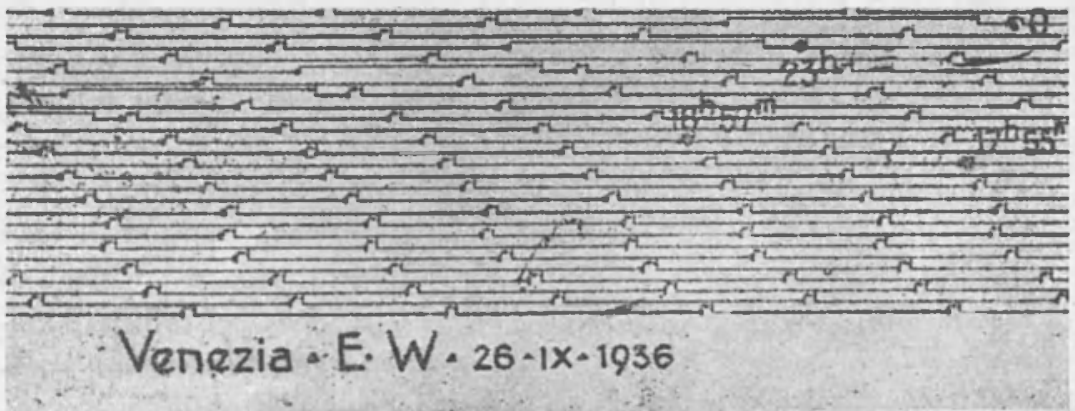

Fig. 35 

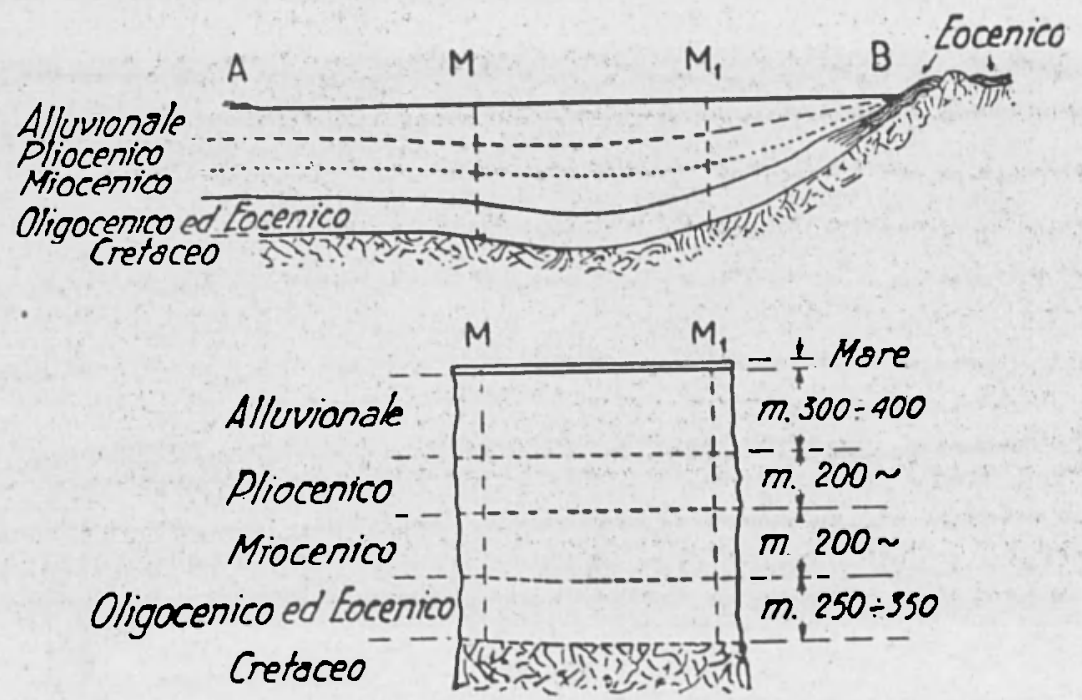

Fig. 36

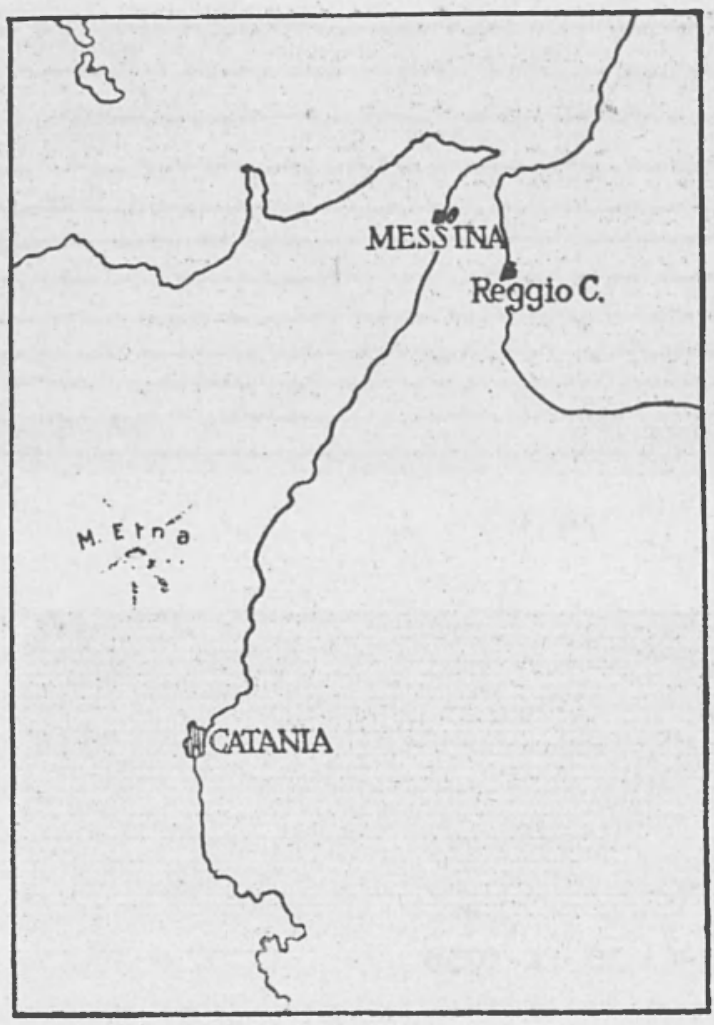

Fig. 37 tenue copertura di detriti al lato orientale della supposta frattura.

Taramelli ritiene, con Spallanzani, che in conseguenza dei moti avvenuti nella zona nel Terziario e nel Quaternario l'area marina fra Sicilia e Calabria sia andata progressivamente restringendosi, per ridursi, da una larghezza originaria di almeno $30 \mathrm{~km}$, " all'attuale solco tortuoso che, con la larghezza minima di ca 4 km, separa i dirupi di rocce cristalline, ammantati da tenue spessore di sedimenti marini quaternari " $\left({ }^{8}\right)$. 


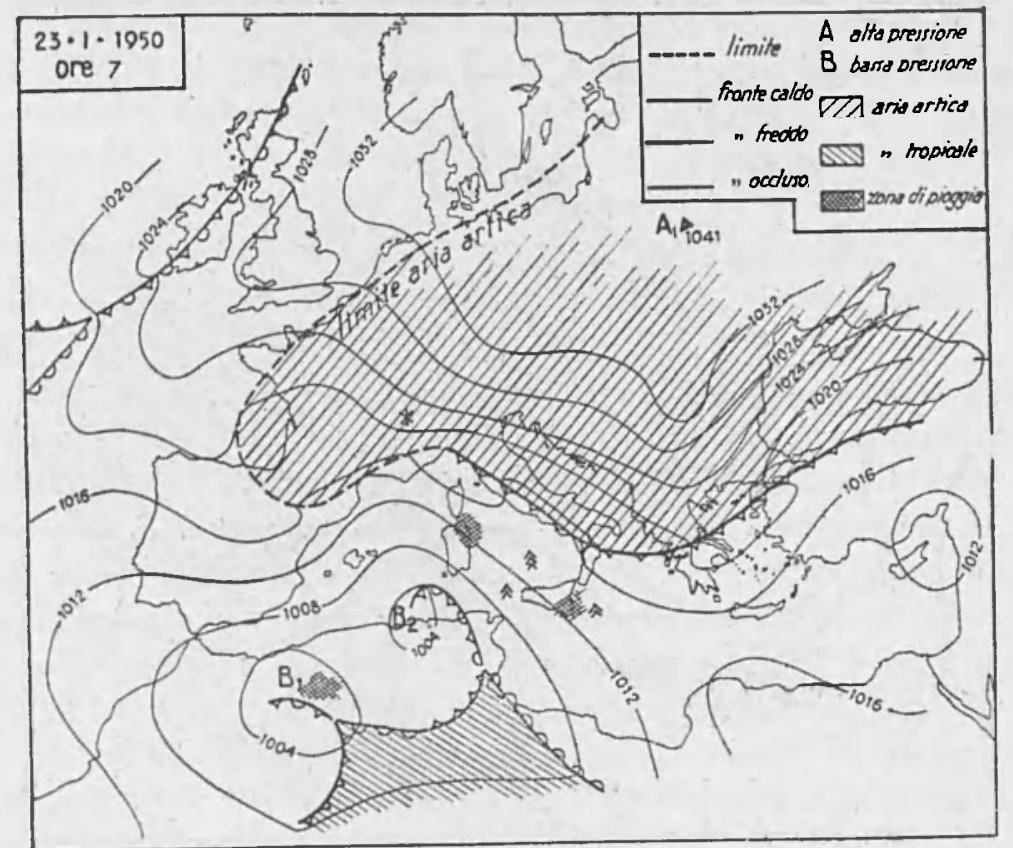

Fig. 38

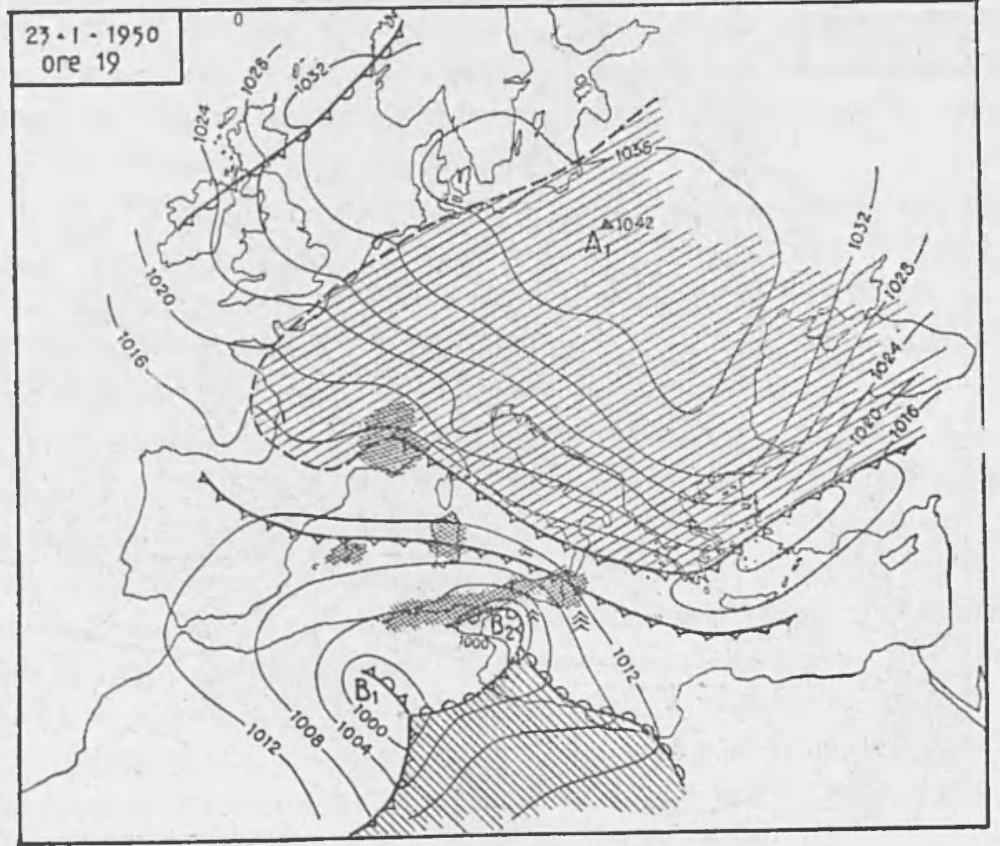

Fig. 39 


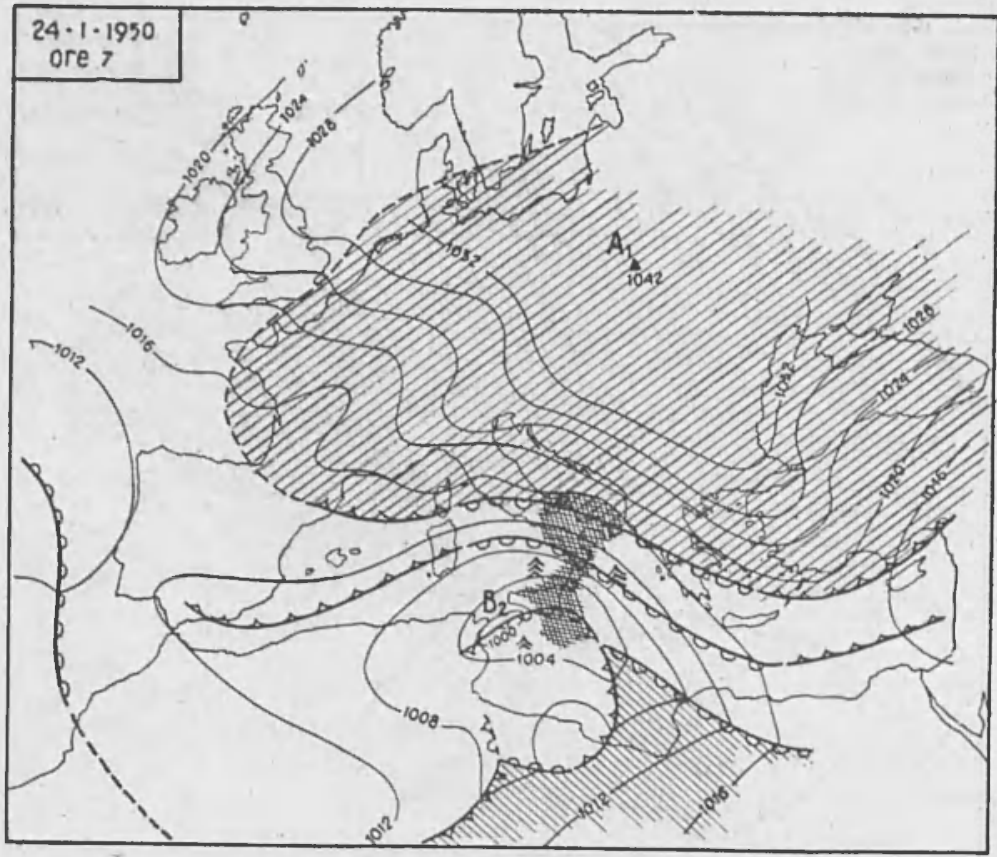

Fig. 40

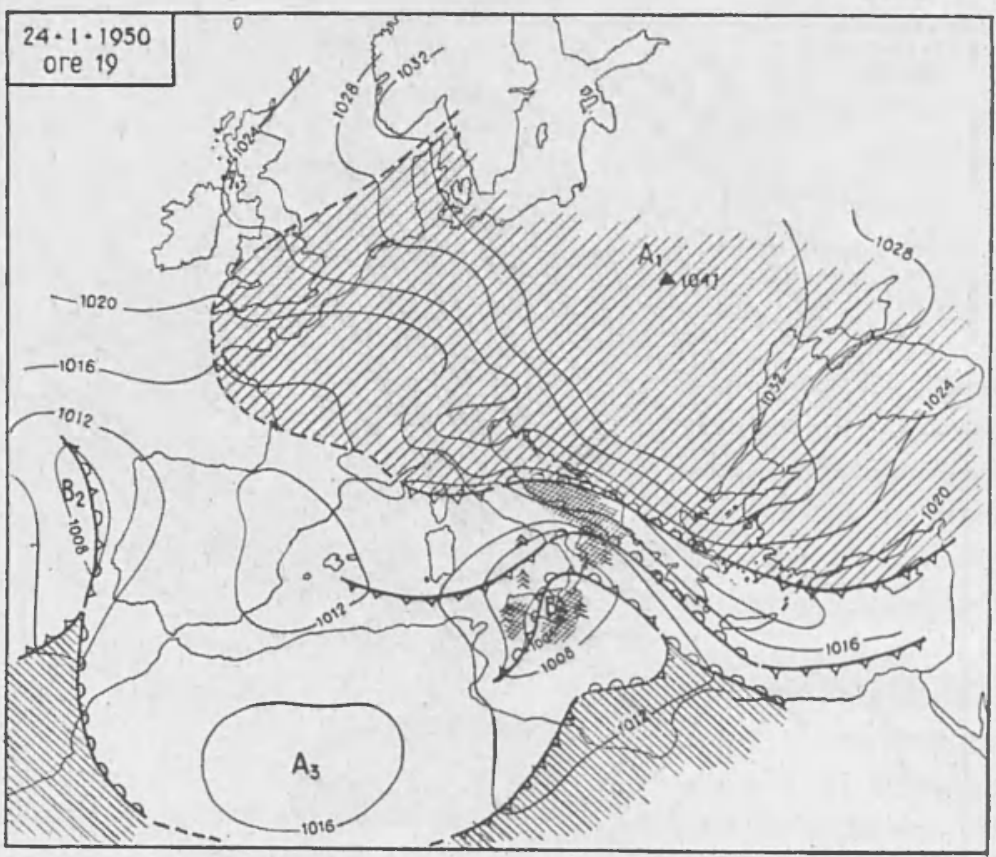

Fig. 41 
" Ma quanto più ristretto si faceva questo braccio di mare, tanto più violenta si manifestava la forza delle correnti di mare, le quali al presente raggiungono la velocità di veri fiumi (oltre a $\mathrm{m} 2$ al minuto secondo) " ( ${ }^{8}$ ). A tali correnti marine è soprattuto da imputare la piccola sedimentazione sul fondo dello stretto.

Ben diversa è la situazione a settentrione dello stretto. A $\mathrm{N}$ dello stretto il fondo del mare presenta un declivio assai dolce, specie dalla parte della costa sicula.

Ivi la sedimentazione è più evidente. Ciò risulta in modo chiaro anche dal confronto fra la batimetria del 1876-1877 (fig. 48) e quella eseguita nel 1909 (fig. 49), dopo il terremoto di Messina ( ${ }^{9}$ ). Da tale confronto "degna di particolare attenzione è la diminuzione di fondali che si nota fuori dello stretto verso $\mathrm{N}$. Le linee dei 200 e dei 300 metri appariscono ora molto più lontane, tanto dalla costa sicula che da quella calabra, di quello che non fossero nel 1877 , e la curva dei 400 metri presso quest'ultima costa ora più non esiste " ( $P$. Marzolo).

A parere concorde di alcuni geologi (Taramelli, Beneo, ecc.) ciò è da attribuire ad " interramento dovuto a deposito di materie dipendente dal gioco delle correnti ” (Marzolo) $\left({ }^{8}\right)$.

A nord dello stretto di Messina, nel Tirreno, il fondo marino risulta quindi costituito da uno strato più o meno cospicuo di sedimenti. Una prova di ciò si ha, indirettamente, nel ben diverso comportamento dei microsismi per perturbazioni meteorologiche provenienti dal Tirreno. Valga un esempio.

Il 17 e il 18 dicembre 1949 la situazione meteorologica era come da figg. 50, 51, 52, 53, 54, 55: si ha cioè una translazione di fronti da NW a SE, in direzione dell'asse del Tirreno, all'incirca. L'andamento della pressione a Messina è riportato nella fig. 56 assieme alla velocità e direzione del vento nella stessa località.

Nel caso precedente, non ostante l'atmosfera e il mare fossero fortemente agitati e a Catania venissero registrati microsismi eccezionalmente ampi, a Messina non si ebbero microsismi apprezzabili. In questo caso, invece, i microsismi, anche se non di notevole ampiezza, sono sensibili (fig. 57). Essi possono senz'altro attribuirsi al transito di rapide perturbazioni microbariche, associate a pressione montante, sulla parte meridionale del Terreno, in modo d asoddisfare alla [3], st non addirittura alla [5]. Il fenomeno però si è realizzato in quanto le perturbazioni atmosferiche, trasferite sul fondo del mare, trovano ivi le condizioni per la loro trasmissione nel mezzo solido. Da qui il ruolo 


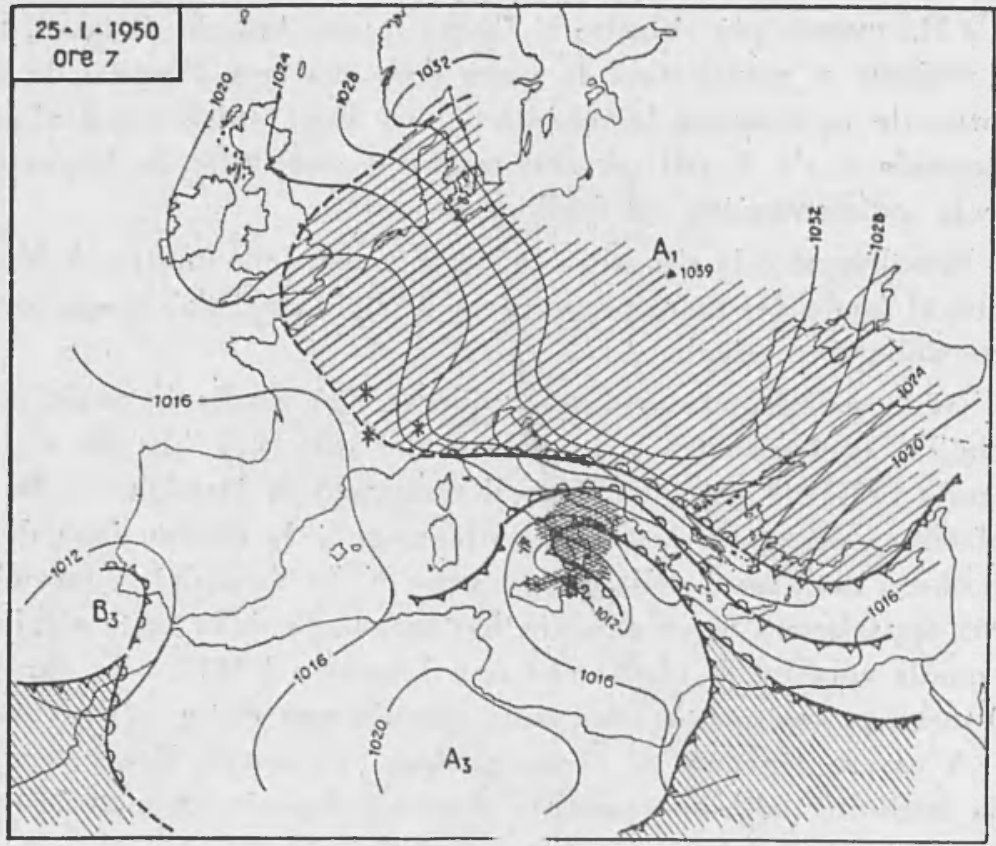

Fig. 42

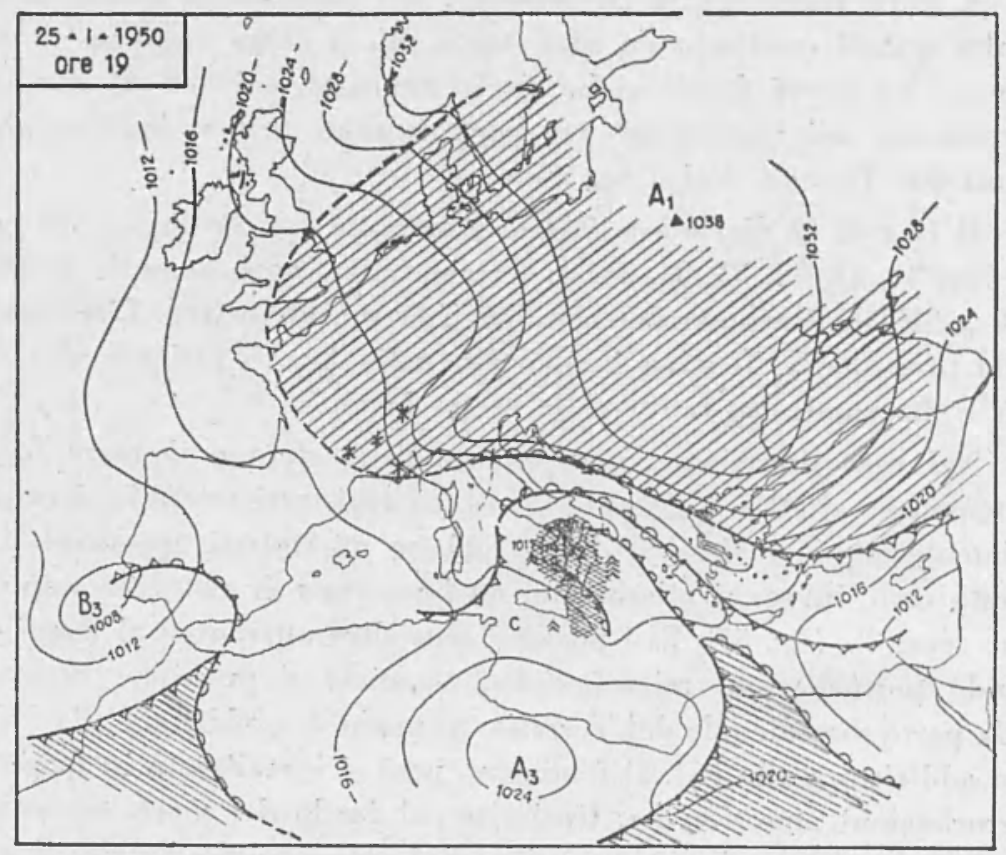

Fig. 43 


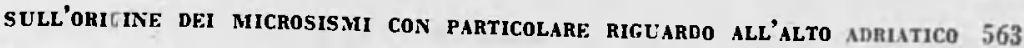

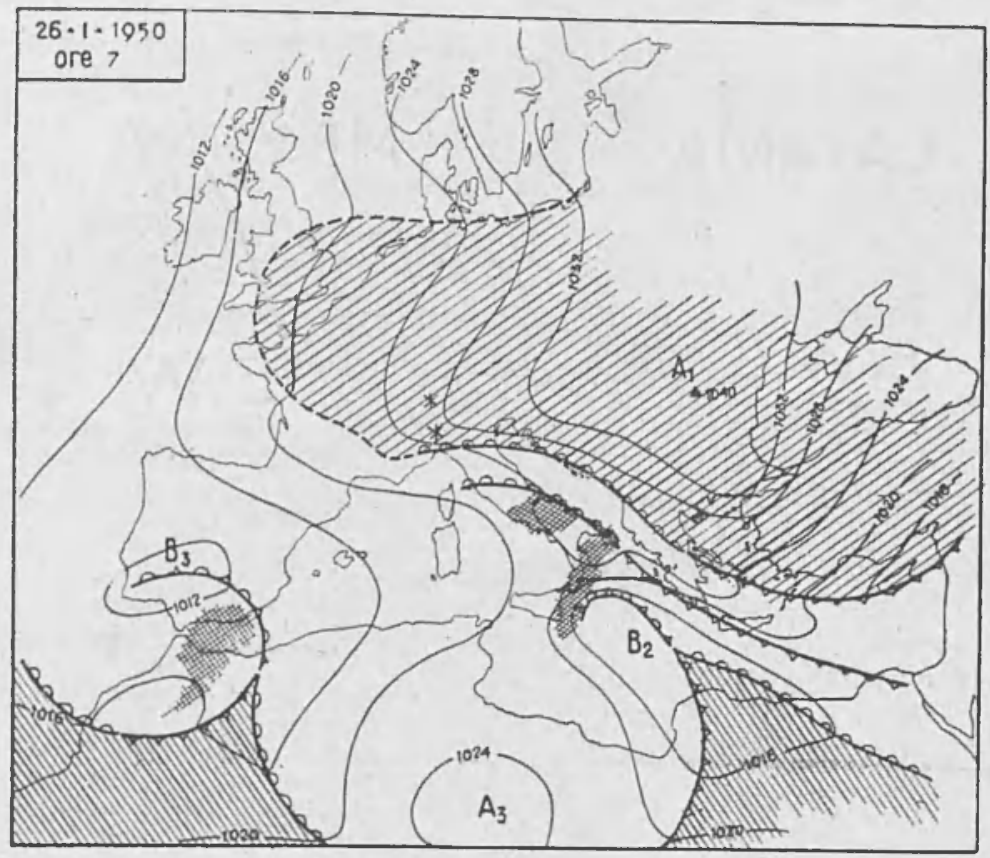

Fig. 44

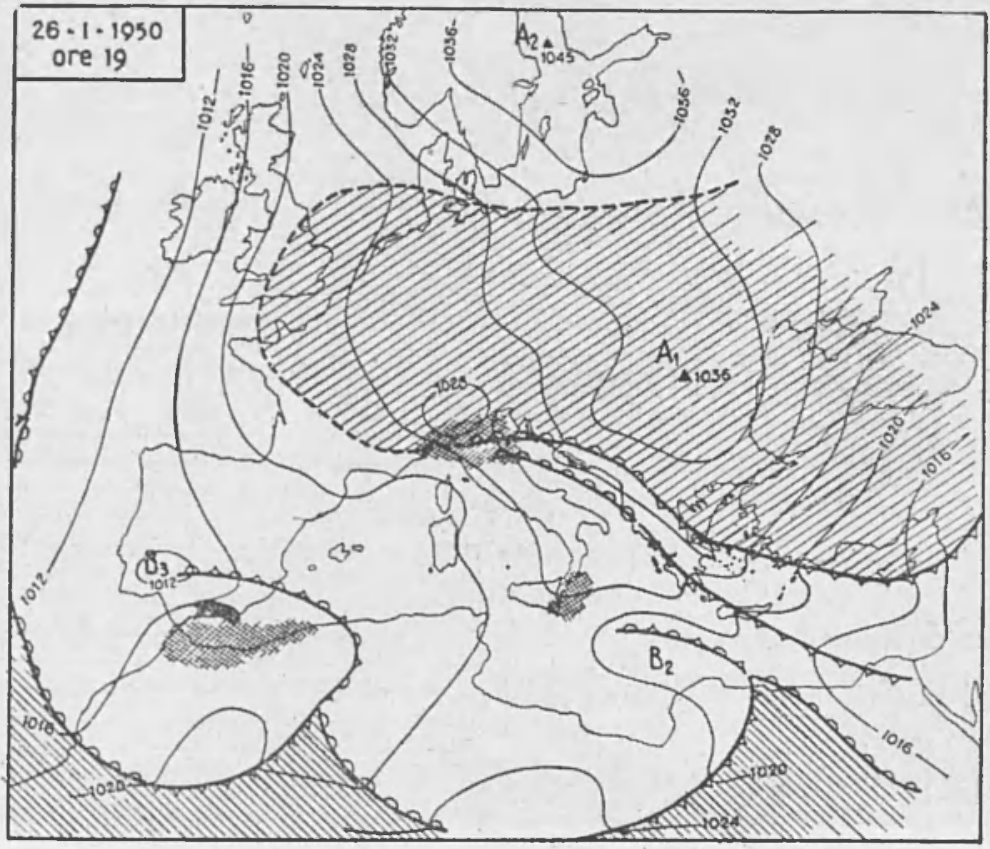

Fig. 15 


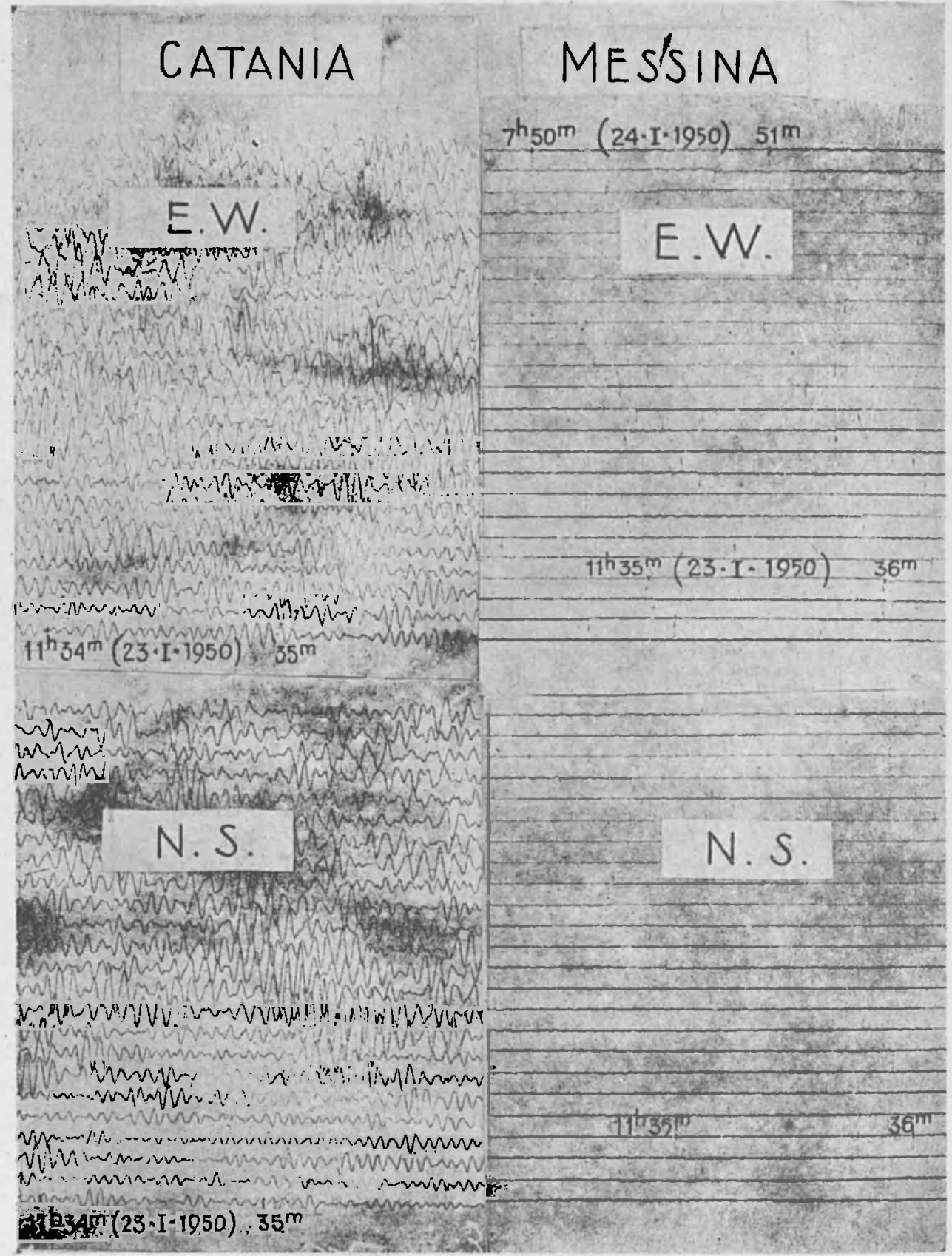

Fig. 46 
essenziale degli strati sedimentari nella formazione e trasmissione dei microsismi.

L'esempio riportato è notevole anche da un altro punto di vista: esso ha fornito, nelle registrazioni di Catania, una chiara testimonianza di microsismi provenienti da due diverse origini. Si è già osservato che la zona di mare intorno a Catania è estremamente sensibile a microsismi causati dal moto ondoso (che probabilmente agisce sul fondo con sistemi ritmici di oscillazioni stazionarie). Mentre a Mes-

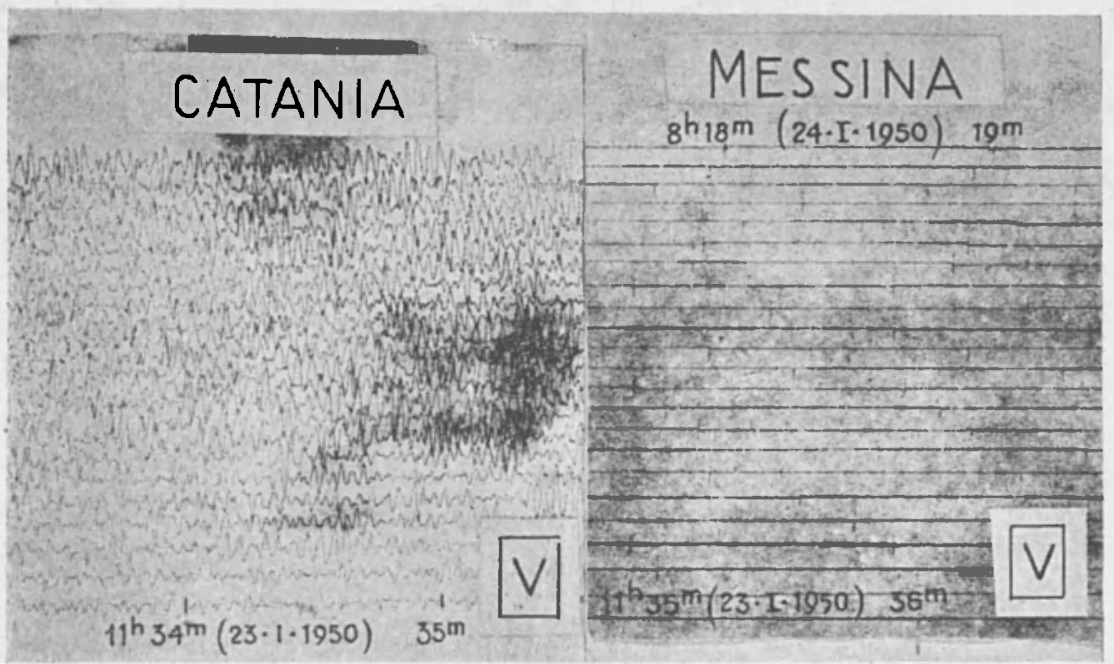

Fig. 47

sina era in corso la lieve agitazione microsismica, dovuta alle rapide perturbazioni di pressione provenienti dal Tirreno, legate alla depressione accennata, a Catania i Wiechert stavano registrando l'abituale agitazione microsismica per mare mosso. L'andamento della depressione è tale (come risulta dalle carte riportate e dai dati barometrici) da far giungere a Catania le perturbazioni rapide dell'atmosfera qualche tempo dopo il loro arrivo a Messina. Il loro transito sul mare di Catania provoca un'agitazione microsismica, che si sovrappone a quella in atto e che da essa si distingue per essere un po' più rapida e per una più spiccata componente verticale. Ciò appare chiaramente dalle registrazioni delle componenti orizzontali (fig. 58) e in modo netto, inequivocabile dalla componente verticale (fig. 59), dove la nuova agitazione, legata al transito delle rapide perturbazioni 


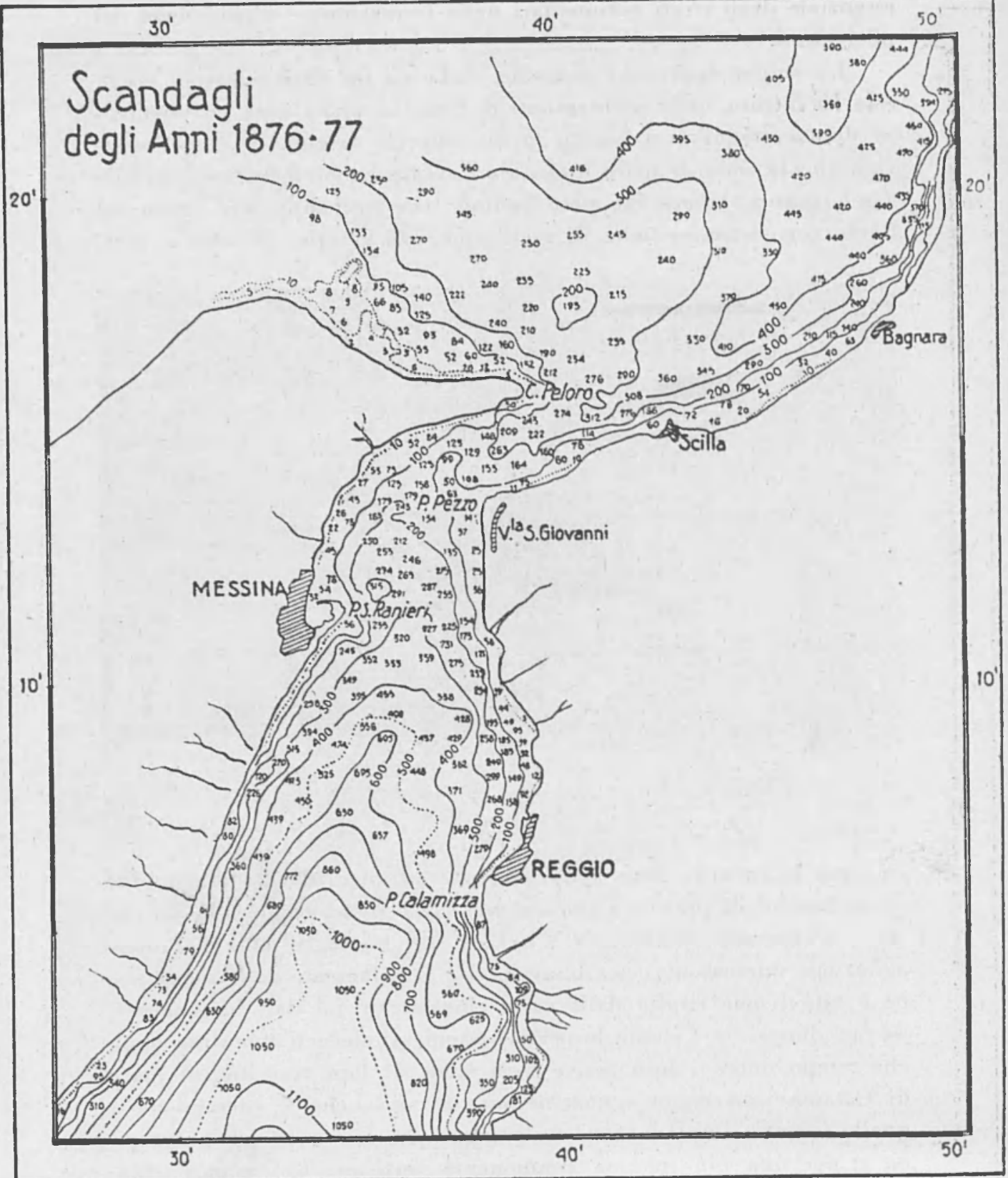

Fig. 48 


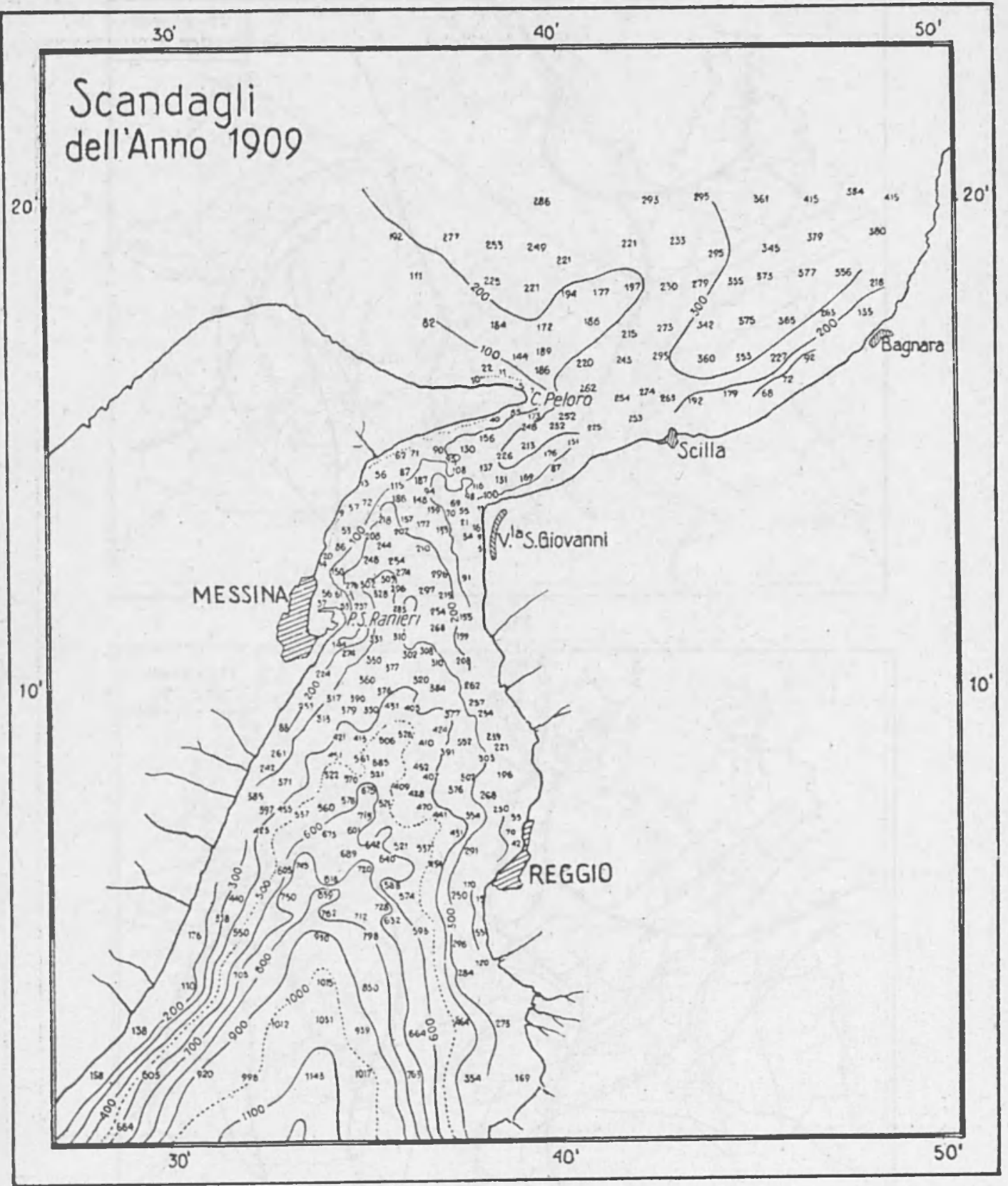

Fig. 19 


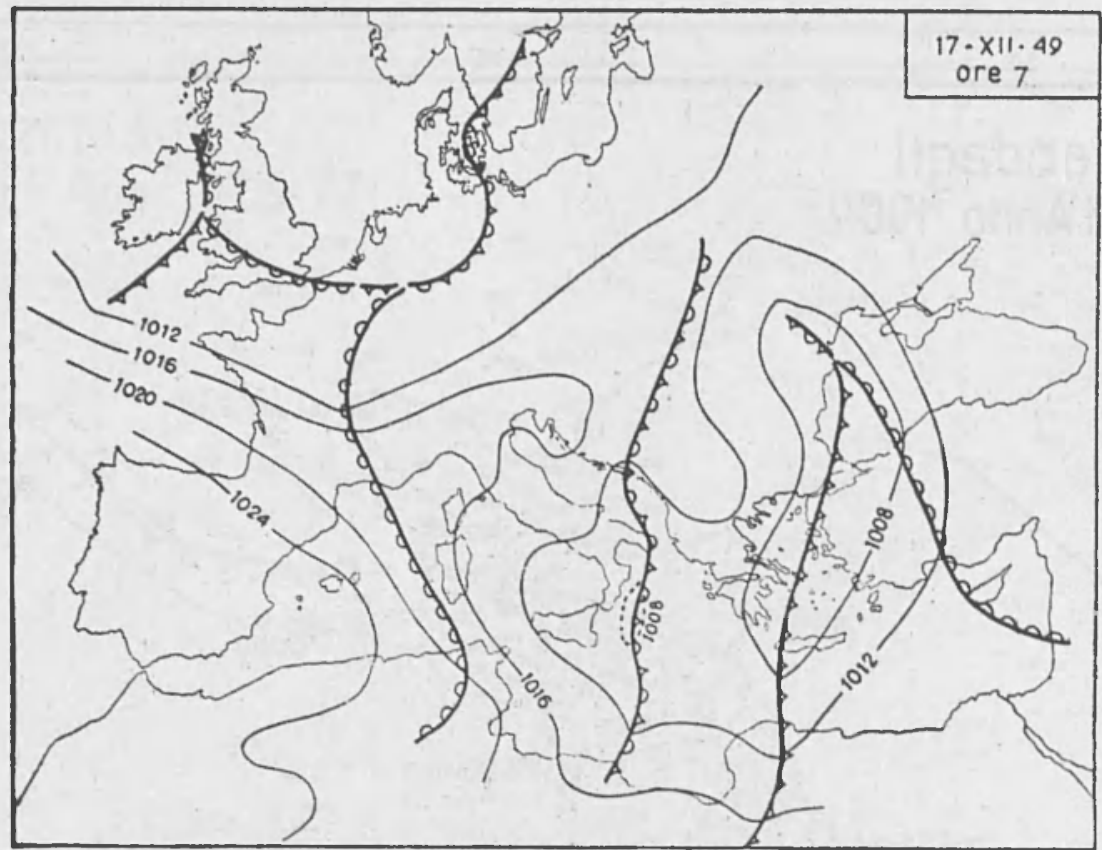

Fig. 50

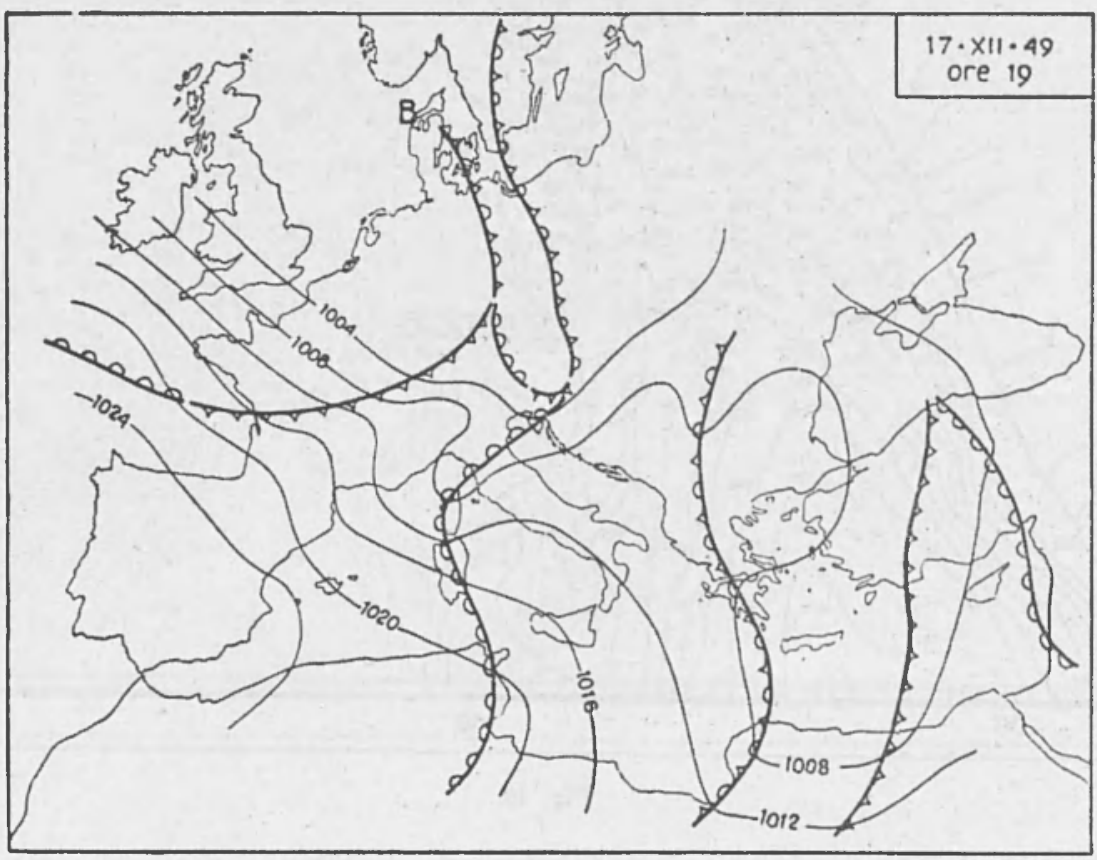

Fig. 51 
SULL'ORIGINE DEI MICROSISMI CON PARTICOLARE RIGUARDO ALL'ALTO ADRIATICo 569

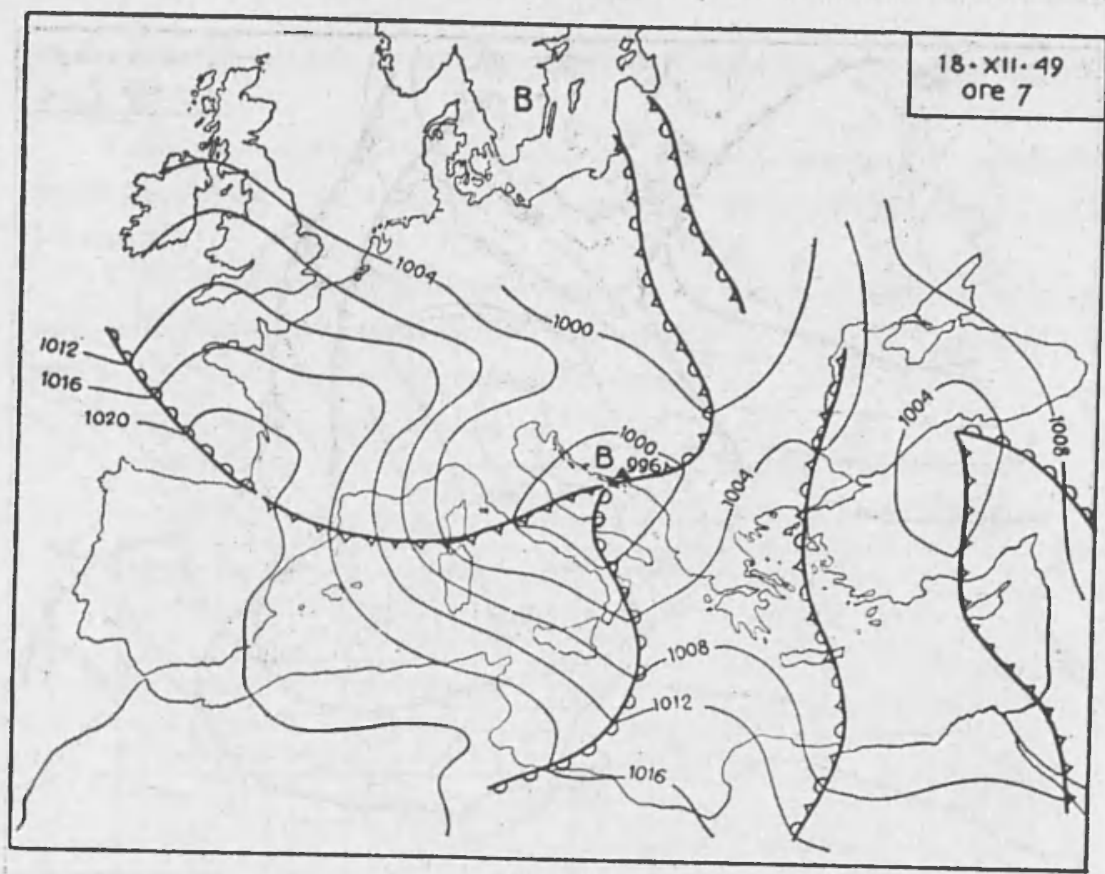

Fig. 52

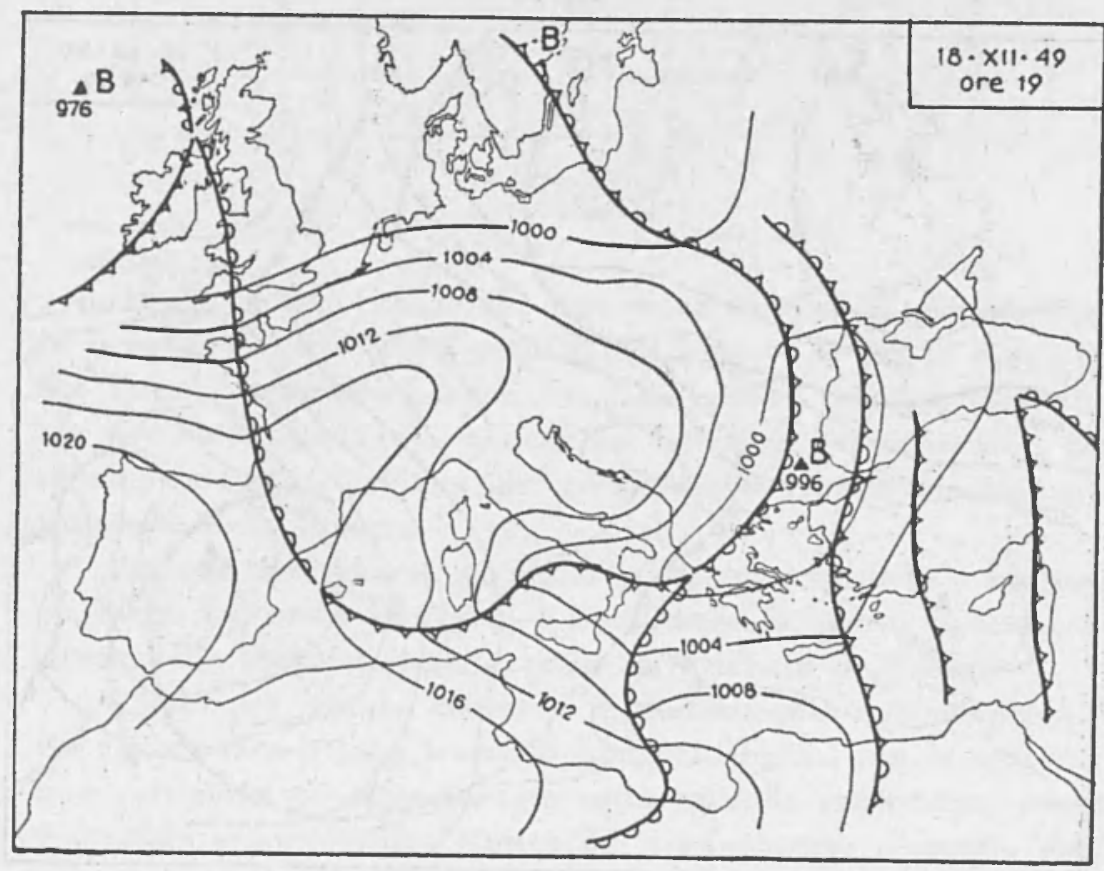

Fig. 53 


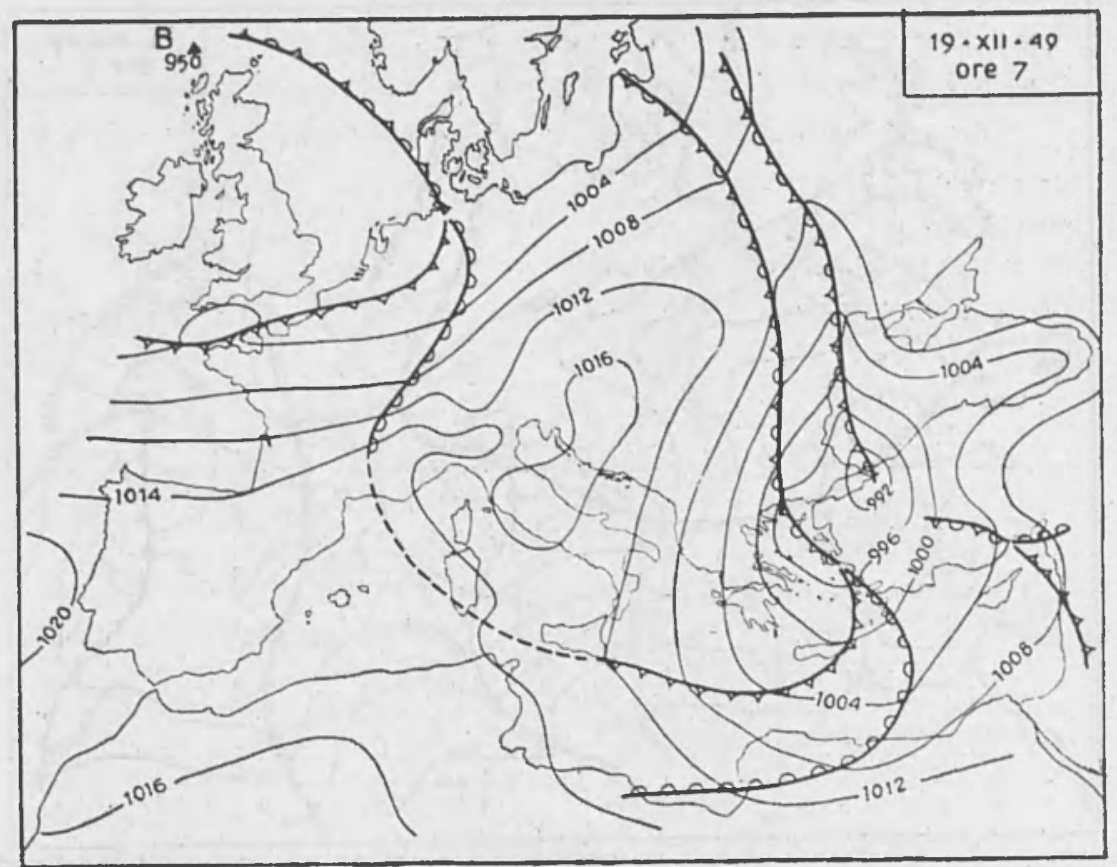

Fig. 54

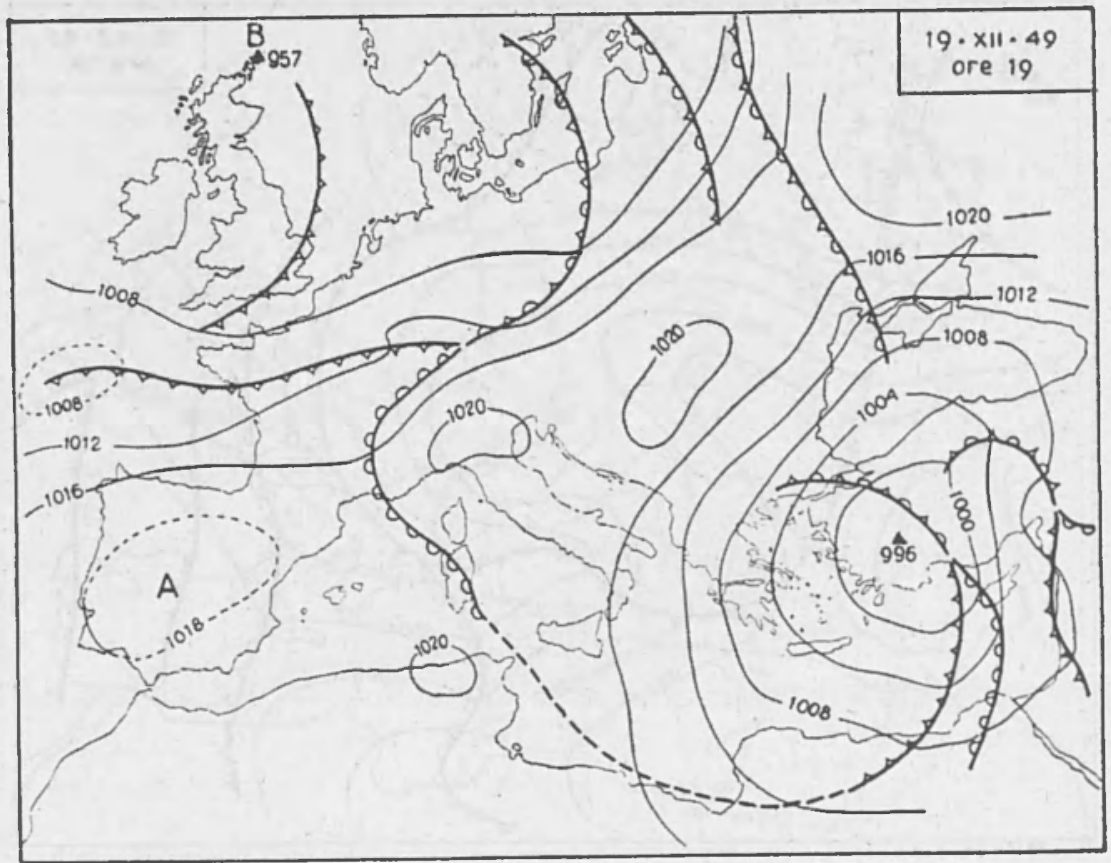

Fig. 55 
atmosferiche, spicca in modo netto sull'agitazione in atto, legata al mare mosso.

Ecco un esempio chiarissimo di sovrapposizione di microsismi, determinati da cause diverse, agenti contemporaneamente e separatamente.

3. - E opinione dello scrivente che la teoria esposta al n. 1 , valevole per l'alto Adriatico, abbia un valore di carattere generale, nel senso che può essere agevolmente estesa ad altri mari e, in certe condizioni anche agli oceani.

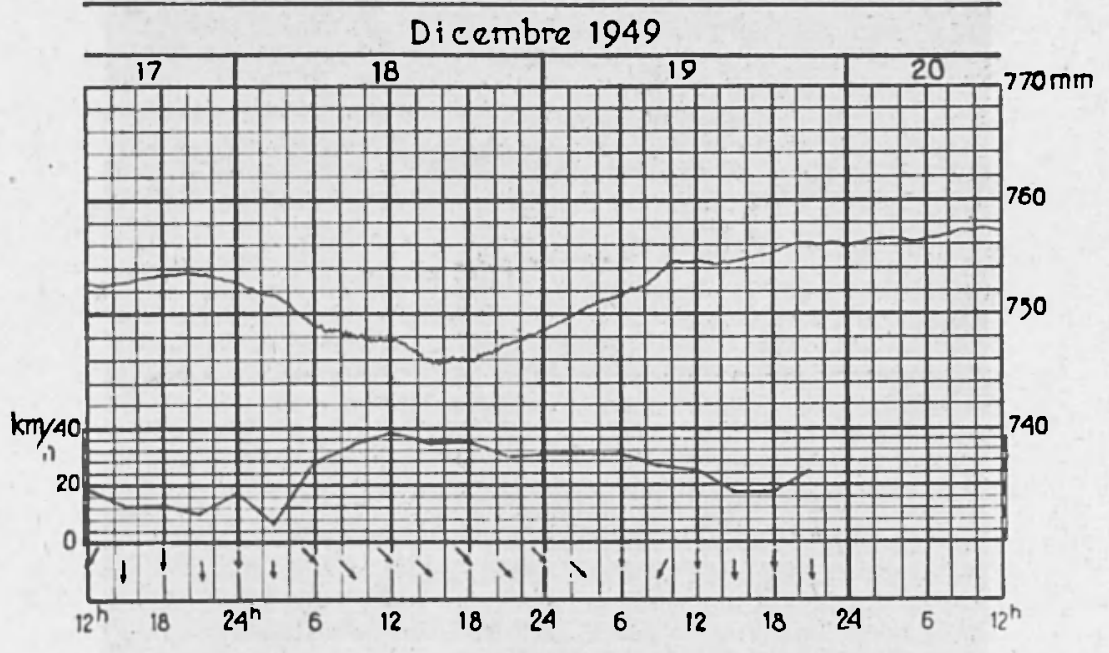

Fig. 56

In particolare, ritengo che essa possa trovare agevole applicazione nel caso dei microsismi generati dal transito di cicloni lungo l'asse del Tirreno (microsismi legati alla fase positiva dei cicloni stessi, come per l'alto Adriatico), nonché le agitazioni microsismiche determinate da nuclei ciclonici che irrompono nel golfo di Genova nel retroterra della Liguria.

Per questa via, forse, potranno essere spiegati anche $i$ microsismi originanti nel mare del Nord: è significativa, a questo riguardo, l'esistenza delle zone privilegiate, messe in evidenza da Lacoste.

Questo per ciò che concerne il trasferimento dell'energia dall'aria agitata al mare. La teoria di Longuet-Higgins, con la scoperta di una variazione di second'ordine sotto un'onda stazionaria, consente d'altronde la spiegazione teorica del trasferimento d'energia dall'acqua al fondo solido. Come nei mari interni possano formarsi onde 


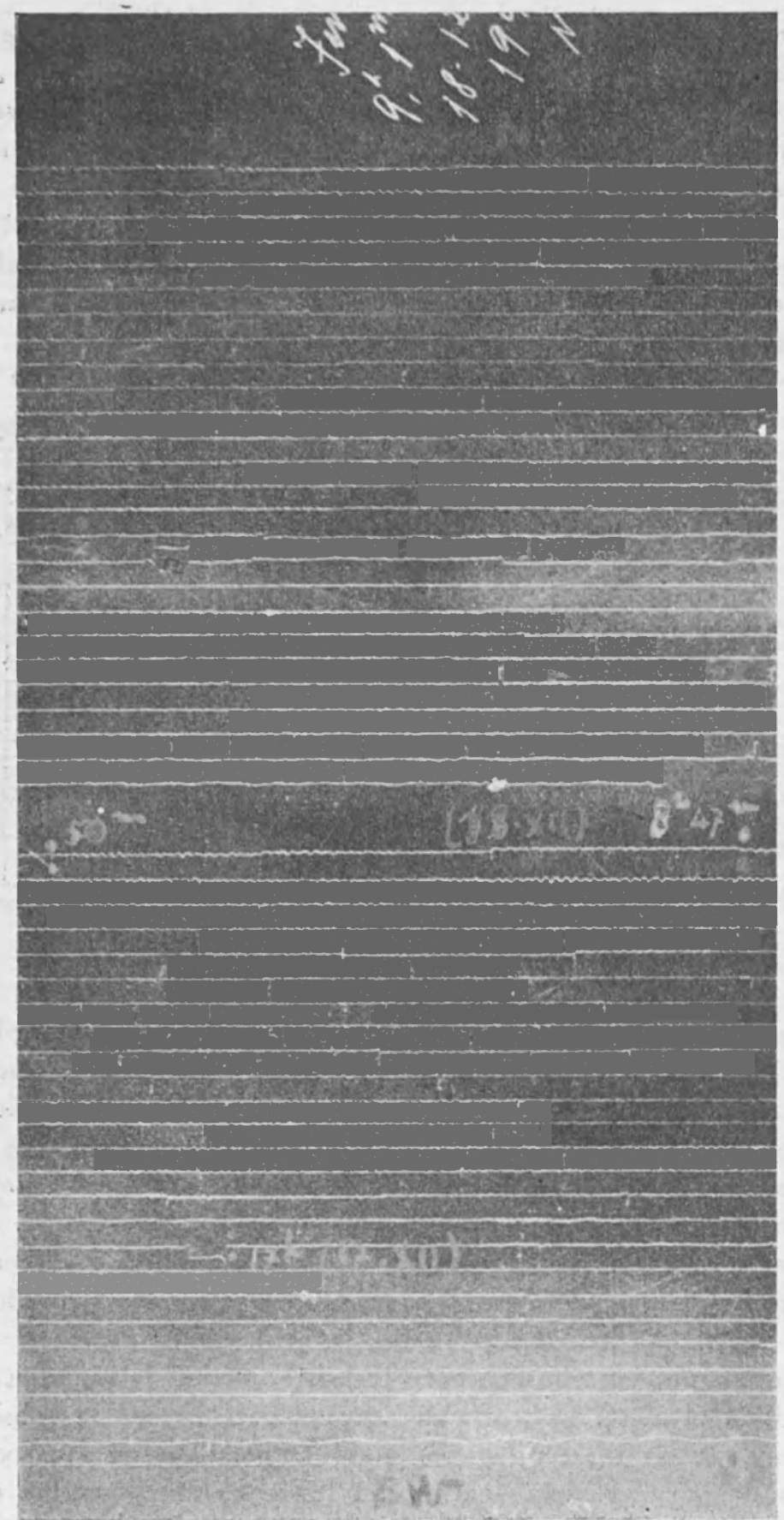

Fig. 57 


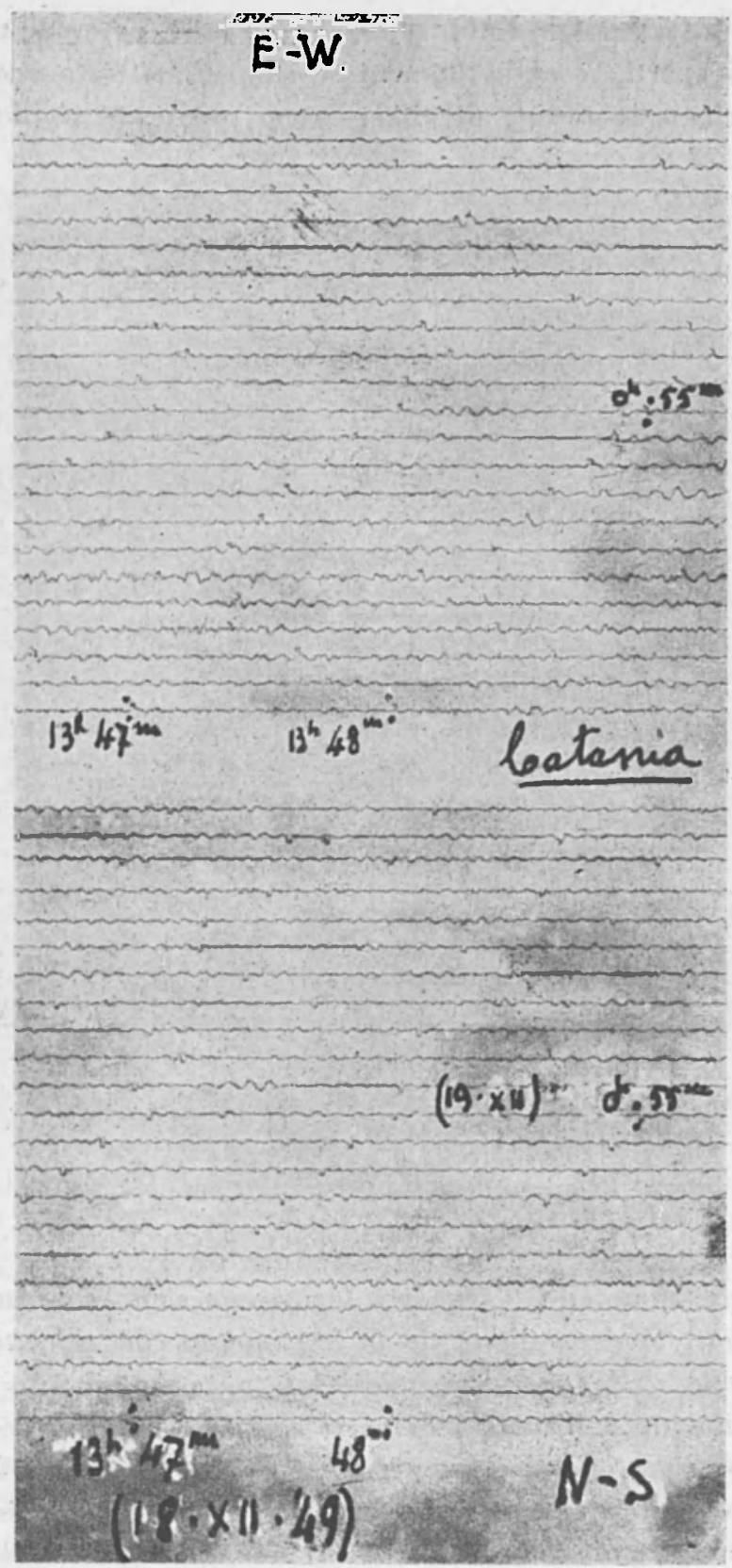

Fig. 58 
stazionarie è facilmente dimostrabile. D'altronde, per quanto si riferisce ai mari aperti, secondo Stoneley, è stato dimostrato che onde stazionarie del giusto ordine di altezza sono provocate vicino al centro

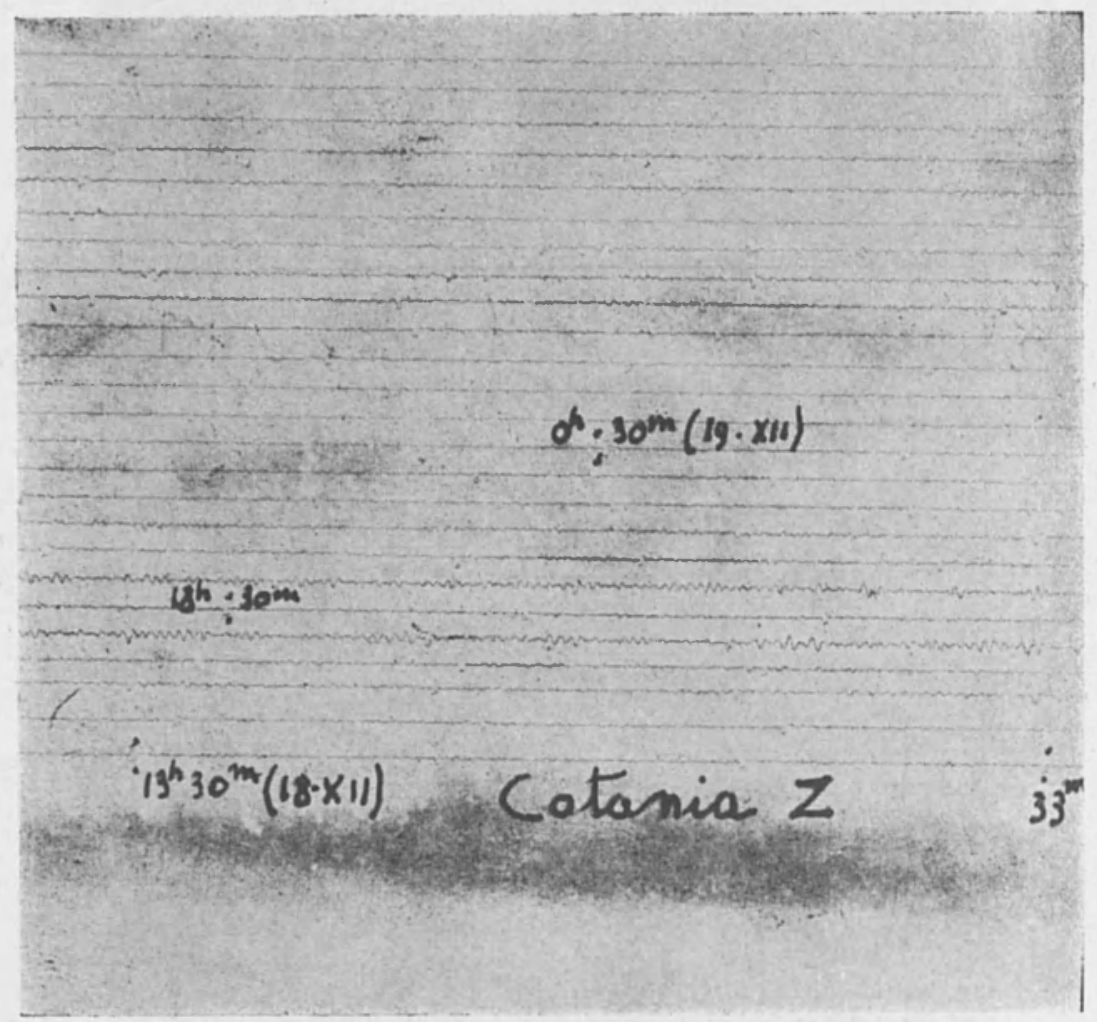

Fig. 59

di una depressione ed $\dot{e}$ anche più logico che siano generate se, come spesso accade, la depressione sia in movimento con velocità comparabile a quella delle onde progressive. $\stackrel{\mathrm{E}}{\mathrm{E}}$ appunto quanto si verifica nel trasferimento dell'energia dall'aria agitata al mare, secondo la teoria di Proudman, che mi ha permesso di provare il perfetto parallelismo tra mare agitato da disturbi microbarici in movimento, con velocità paragonabile alle onde libere, e microsismi. Osserviamo, a questo proposito, che la funzione $F$ nella [3] è funzione qualunque del suo argomento e che la teoria esposta al $\mathrm{n}$. $1 \mathrm{mi} \grave{e}$ servita, fino dal 1938 , per provare, in modo inequivocabile, la formazione delle 
sesse nel golfo di Trieste e nell'alto Adriatico; ed è ben noto che le sesse sono appunto onde stazionarie (*).

$$
\text { Roma - Istituto Nazionale di Geofisica - Luglio } 1951 .
$$

\section{RIASSUNTO}

Già dal 1936 avevo provato che, nel transito dei cicloni sull'alto Adriatico, le rapide variazioni di pressione collegate a detti cicloni e, specialmente, alla fase positiva montante degli stessi, davano origine alla formazione di microsismi, tanto più sensibili quanto più prossima alla velocità delle onde libere del mare sottostante è la velocità dei disturbi microbarici.

L'ampiezza dei microsismi diventa particolarmente notevole quando, sul golfo di Trieste in direzione del suo asse $e$ in senso contrario a quello secondo cui crescono le distanze dalla sua base, transitano rapide variazioni della pressione, collegate oppure no a nuclei ciclonici, con velocità paragonabili a quella delle onde libere del mare. L'effetto è massimo per nuclei a tendenza positiva, collegati a cicloni in transito.

Gli esempi inconfondibili raccolti - alcuni dei quali sono stati qui riportati - danno a questi fatti un'evidenza inconfutabile, quale spetta alle cose certe.

C'è quindi un trasferimento di energia dall'atmosfera agitata al mare e da questo al fondo solido.

L'estensione di una teoria di Proudman sugli effetti della propagazione di variazioni di pressione sul mare, consente di spiegare, in modo chiarissimo, il meccanismo del trasferimento d'energia dall'atmosfera al mare.

Per quanto concerne l'alto Adriatico, per la spiegazione di come avviene il trasferimento dell'energia dal mare agitato al fondo, non è certo necessario fare ricorso a particolari teorie: data la piccola profondità dell'alto Adriatico, le classiche teorie idrodinamiche sono suf-

* Devo ringraziare il personale direttivo dell'Osservatorio Geofisico Patriarcale di Venezia e dell'Istituto Talassografico di Trieste per aver cortesemente messo a mia disposizione numerose registrazioni sismiche, meteorologiche e mareografiche. Un ringraziamento particolare devo al Cap. Fdmondo Bernacca dell'Ispettorato Telecomunicazioni - Servizio Meteorologico - del Ministero dell'Aeronauti$\mathrm{ea}$, che ha tracciato le situazioni meteorologiche, riportate nelle figg. 14-19, 38-45, 50-55. 
ficienti a spiegare tale trasferimento. Queste teorie però cadono in difetto quando la profondità del mare supera il centinaio di metri. $\grave{E}$ qui dove assume invece eccezionale interesse la teoria di LonguetHiggins, che prevede una variazione di second'ordine della pressione sotto un'onda stazionaria. A mio avviso, tale teoria viene a completare il chiarimento teorico dei fatti osservati, colmando la lacuna relativa al trasferimento dell'energia dal mare in agitazione al fondo solido.

D'altronde, come scrive Stoneley, " actually, it is shown that standing waves of the correct order of height will he set up near the centre of a depression, and it is even more likely that they will he generated if, as often happens, the depression is moving with a velocity conparable with that of the progressive waves". E appunto quanto si verifica nel trasferimento dell'energia dallaria agitata al mare, secondo la teoria che mi ha permesso di provare il perfetto parallelismo tra mare agitato da disturbi microbarici in movimento, con velocità comparabile a quella delle onde libere, e microsismi. Aggiungo che tale teoria mi ha consentito di provare, fino dal 1938, la genesi delle sesse nel golfo di Trieste e nell'alto Adriatico; ed è ben noto che le sesse sono appunto onde stazionarie.

$\dot{E}$ molto probabile che tale teoria possa essere agevolmente estesa ad altri mari e, in certe condizioni, agli oceani. In particolare, con essa si possono spiegare $i$ microsismi generati dal transito di cicloni lungo l'asse del Tirreno, nonché le agitazioni microsismiche determinate da nuclei ciclonici, particolarmente quelli legati alla pressione in fase positiva, che irrompono dal golfo di Genova nel retroterra della. Liguria.

Ritengo inoltre che, detta teoria, valg" anche a spiegare la creazione dei microsismi da parte dei sistemi d'onde, provocati alla superficie del mare da vento soffiante in una stessa direzione, con velocità pressoché costante.

Con ciò viene isolata e chiarita una delle cause fondamentali se non la fondamentale - a cui vanno at tribuiti $i$ microsismi.

$V a$ subito aggiunto però che, quanto ì stato fin qui detto costituisce la condizione necessaria, ma non sufjiciente, per la creazione di microsismi di apprezzabile ampiezza. La natura geologica del fondo del mare giuoca, a questo riguardo, un ruolo di primaria importanza: per la formazione e la propagazione dei microsismi a grandi distanze $\grave{e}$ infatti necessario che il fondo del mare consist"n di stratificazioni atte a ricevere e ad esaltare gli impulsi energetici che ad esso proven- 
gono attraverso l'acqua del mare. Il ben diverso comportamento dell'alto Adriatico e dello stretto di Messina sotto l'azione di analoghe cause agenti, costituisce una chiara testimonianza della validità di questa condizione.

\section{BIBLIOGRAFIA}

(1) Calor P.: Oscillazioni del mare e perturbazioni della verticale apparente nel golfo di Trieste durante il rapido transitare di alcuni cicloni attraverso l'alto Adriatico. Atti Ist. Ven., 1935-1936, XCV.

(2) Proudman J.: The Effects on the Sea of Changes in Atmospheric Pressure. Roy. Astr. Soc., Geophys. Suppl, II. 4 (1929).

(3) Calor P.: Sesse dell'alto Adriatico con particolare riguardo al golfo di Trieste. Com. Talass. It., Mem. CCXLVII (1938).

(4) Morelli C.: Contributo allo studio dei microsismi. Annali di Geofisica, I, 4 (1948).

(5) Andreotti G.: Ulteriori studi su alcune agitazioni del suolo a Padova. Atti Ist. Veneto, XCI (1932).

(6) Andreottr G.: Indagini sulla via di propagazione delle perturbazioni microsismiche fra Trieste e Padova. Atti Ist. Veneto, XCI (1932).

(7) Calor P.: Due caratteristici tipi di microsismi. Annali di Geofisica, III, 3 (1950).

(8) Relazione della Commissione Reale sugli effetti del terremoto del 28 dicembre 1908, Roma (1909), pag. 49.

(9) Ibid. Allegato B, pag. 113. 